Portland State University

PDXScholar

Fall 11-16-2018

\title{
Shear-Force Acoustic Near-Field Microscopy and Its Implementation in the Study of Confined Mesoscopic Fluids
}

Theodore Alex Brockman

Portland State University

Follow this and additional works at: https://pdxscholar.library.pdx.edu/open_access_etds

Part of the Physics Commons

Let us know how access to this document benefits you.

Recommended Citation

Brockman, Theodore Alex, "Shear-Force Acoustic Near-Field Microscopy and Its Implementation in the Study of Confined Mesoscopic Fluids" (2018). Dissertations and Theses. Paper 4710.

https://doi.org/10.15760/etd.6594

This Thesis is brought to you for free and open access. It has been accepted for inclusion in Dissertations and Theses by an authorized administrator of PDXScholar. Please contact us if we can make this document more accessible: pdxscholar@pdx.edu. 


\title{
Shear-Force Acoustic Near-Field Microscopy and Its Implementation in the Study of Confined Mesoscopic Fluids
}

by

Theodore Alex Brockman

A thesis submitted in partial fulfillment of the requirements for the degree of

\author{
Master of Science \\ in \\ Physics
}

Thesis Committee:

Andres La Rosa, Chair

Erik Sánchez

Shankar Rananavare

Portland State University

2018 
(C) 2018 Theodore Alex Brockman 


\begin{abstract}
The recently developed Shear-Force Acoustic Near-Field Microscope (SANM) is used to investigate the viscoelastic properties of a mesoscopic fluid layer confined between two trapping boundaries, one being a stationary substrate and the other the apex of a laterally oscillating tapered probe. Hardware improvements and evaluation of the SANMprobe robustness will be a major focus of this thesis. The investigation first discusses characterization and recent developments made to the microscope, including: modifications to the sensor head, conditioning of the nano positioners electrical drive signal, and the assessment of the probe against eventual plastic deformation or compliance against interactions with samples, (the latter comprising a solid substrate and its adhered fluid layer which is typically a few monolayers thick). Furthermore, this study includes an analysis of the adsorbed mesoscopic fluid's viscoelastic properties. This inquiry aims to better understand probe-sample interactions with the mesoscopic fluid. This includes adhesion [1] [2], wetting [3], and to inquire the nature of the hydrophobic interaction, which is relevant in many areas of study such a protein folding [4], and interfacial friction which has wide ranging applications including desalination [5].This analysis will be performed using a Sheer force microscopy (implemented with quartz tuning fork QTF), and another recently introduced technique Whispering Gallery Acoustic Sensor (WGAS). The latter allows more direct monitoring of the QTF's mechanical displacement. These measurements will be supplemented by simultaneously monitoring the acoustic emission from the mesoscopic fluid under confinement between the probe and the substrate, which will be monitored using the SANM sensor positioned beneath the substrate.
\end{abstract}




\section{Acknowledgements}

This work could not have been made possible without the support and guidance of many individuals, I would like to take this opportunity to express my sincere gratitude. First, I would like to thank my committee members: Andres La Rosa, Erik Sanchez, Shankar Rananavare. I know there are more entertaining ways to spend your time than reading a master's thesis, and appreciate you all being part of my academic development. Furthermore, I would like to thank my friend and colleague Monte for the comradery, and constant willingness to bounce ideas around. I would also like to thank Jianghua for always being willing share and to teach your wealth of knowledge on circuits and control theory. I would also like to thank Rodolfo for being a mentor.

I would additionally like to recognize Alex, and Marc, for their assistance in creating multiple microscope components, and enthusiastically teaching me to improve my own machining work. My gratitude also extends to Leroy for troubleshooting and repairing our most complex electrical systems when they fail at the least opportune moments. Foremost, I would like to thank my teacher and advisor Andres La Rosa for your guidance, the opportunity to work in the lab, and your continued support of my studies,

Most of all, I would like to thank my parents and sister for the continued encouragement and inspiration,

Finally, I would like to thank Madison. You put up with my questionable humor,

and your endless support and optimism keeps me motivated and reminds me to find enjoyment in the little things. 
Table of Contents

Abstract___ i

Acknowledgements __ ii

List of Figures _

List of Abbreviations __ $\mathrm{xi}$

Chapter 1

Introduction _ 1

(1.1) Introduction and Background on Proximal Probe Microscopy __ 1

(1.2) The Adaption of Tuning Fork Sensors in Probe Microscopy _ 5

(1.3) Sheer Force Acoustic Near-Field Microscopy ___ 6

(1.4) Interfacial Shear Force Microscopy ___ 7

Chapter 2

Experimental Setup

(2.1) The Tuning Fork Based Sensor__ 10

(2.2) QTF Tapered Gold Probe Fabrication __ 12

(2.3) QTF/AFM Probe Fabrication __ 16

(2.4) The Tuning Fork Sensor: Theoretical Basis and Calibration 19

(2.5) Decoupling Forces: The Need for Frequency Modulation 25

(2.6) Retrieving Resonance Frequency Shift and Resonance Frequency Amplitude

from Constant Frequency Measurements 27

(2.7) Frequency Modulation Method__ 28

(2.8) Acoustic Sensors 30

(2.9) The WGAS Technique _ 32

(2.10) The SANM Technique 37

(2.11) Acoustic Sensor Characterization_ 38

(2.12) Control of the Probe's Vertical Position 39

(2.13) Incorporating a Sallen-key Filter into the QTF's Driving Voltage___ 41

(2.14) Operating a Strain Gauge Differential Amplifier $\quad 48$

(2.15) Construction of a Humidity Chamber with Added Salts to Establish Fixed

Points of Humidity Levels

(2.16) Use of Stepper Motors to Control the Probes Coarse Approach $\quad 52$

(2.17) Configuration for Measuring Probe-Substrate Tunneling Current 54

(2.18) Sensors' Electrical Noise Considerations 56

Chapter 3

Results__ 60

(3.1) Interactions with the Adsorbed Water Layer 60

(3.2) A Note on Surface Hydrophobicity and Sample Preparation 61

(3.3) Monitoring Probe Deformation. Case of Tapered Gold Probes: 64

(3.4) Amplitude Dependent Compliance Experiment___ 68

(3.5) Monitoring Acoustic Emission from Confined Mesoscopic Fluids __ 75 
(3.6) Hydrophobic vs Hydrophilic Sample Response

(3.7) Evolution of Approach Curves Under Increasing Probe Sample Interactions 83

(3.8) Next Steps 85

(3.9) Conclusions__ 89

Works Cited

Appendix A 95

Derivation of the effective spring constant of a cantilever

Appendix B 98

Derivation of the effective mass of a cantilever __ 98

Appendix C_ 101

Deriving the Mechanical Amplitude and Phase lag of a Quartz Tuning Fork __ 101 from its Electrical Response.

Appendix D__ 103

Derivation of Tuning Fork -Sample Interaction Forces___ 103

(Case: Frequency Modulation)__ 103

Appendix E_ 106

Derivation of mesoscopic fluid's damping and elastic coefficients 106

(Case: constant frequency) __ 106

Appendix F 109

Calculating Power Spectral Density ___ 109

Appendix G_ 112

A Note on Synchronous Detection with Lock-In amplifier __ 112 


\section{List of Figures}

Figure 1 Partially exploded diagram of the individual components that constitute the microscope used in this thesis. a) Humidity chamber, b) WGAS acoustic sensor, c) tuning fork fixture assembly, c.1) tuning fork fixture, c.2) collet chuck, c.3) Macor with tuning fork installed, c.4) collet clamp, d) stepper motor, e) stepper motor drive coupler assembly, f) whispering gallery acoustic cavity, g) SPM base plate, h) SANM acoustic sensor, i) microscope base incorporating piezoelectric positioners.

\section{9}

Figure 2 The QTF still in its protective 'can' housing is displayed in a). b) displays the tuning fork after its been removed from the can using the side cutter technique, note the epoxy has been crushed (visible above the TF) and no ring is present. c) displaying the canopener technique where can is cut away, leaving the epoxy ring attached to the oscillator.

Figure 3 Probe fabrication via chemical etching. Left) Diagram showing the metallic tip etching setup with inset displaying close-up view of critical elements. a) z positioner, b) anode wire connection, c) gold etching wire, d) graphite cathode, holder and electrical connection, e) pipet bubble guide, (green scale bar $\sim 8.5 \mathrm{~cm}$ ). Right) Electrical schematic of etching power supply including unipolar to bipolar supply converter (with voltage follower to decouple the circuit impedance from the divider), Summing amplifier inside the Stanford Research Function Generator, and non-inverting amplifier with gain of 2 . Note the schematic is presented in a simplified diagram. i) depicts a circuit used to convert a single ended supply into a bipolar supply for the op-amps, in turn creating a virtual ground that can be used by ii) and iii). ii) depicts an adding amplifier taking both the DC etch bias and the etch AC, and outputting a single signal at 'Etch Signal'. Finally, this 'Etch Signal' form ii) is fed into the 'Etch Signal' at the non-inverting input of iii) before being fed to the gold anode though the follower circuit at 'Etch Output'. 13

Figure 4 Left) SEM image of gold tips setting on carbon tape after being etched and cleaved from the wire, (green scale bar $=1.25 \mathrm{~mm}$ ). Right) SEM image of a gold probe after being epoxied to a tuning fork (green scale bar $=650 \mathrm{um}$ ).

Figure 5 Left) Tuning fork spectral response and quality factor corresponding to different added mass. Right) SEM image of the tuning fork after the addition of the $3^{\text {rd }}$ mass (green scale bar $=450 \mathrm{um})$. 16

Figure 6 Diagram of the 5-axis apparatus used to mount AFM cantilevers to the bottom of tuning fork, (green scale bar $\sim 4 \mathrm{~cm}$ )). Apparatus composed of a) jig, b) Thor Labs 3 axis positioner, c) mounting arm. (Note, the Thor Labs 3 axis positioner component modeled in this caption is from Thor Labs product guide). 18

Figure 7 Left, SEM image of AFM cantilever after being epoxied to the bottom of a tuning fork, (green scale bar $\sim 70 \mathrm{um}$ ). Right. Close image of the AFM probe illustrating the geometry (green scale bar $\sim 10 \mathrm{um}$ ).

Figure 8 Left) Schematic of quartz tuning fork with zero applied driving voltage Right) Schematic of quartz tuning fork at time $t$, with tines exibiting deformation caused by applied driving voltage $\mathrm{V}$ 
Figure 9 Left)Schematic representation of quarts tuning fork Butterworth-Van Dyke electrical equivelence citcuit Right) Schematic representation of quartz tuning fork as simple harmonic oscilator, with equivalent mass Meff and spring constant keff. 20

Figure 10 A Plot of the modeled tuning fork current Ytotal $\omega / \mathrm{V}$ (blue) along with measured current (using lock in amplifier) to determine fit parameters. 23

Figure 11 A plot of the modeled tuning fork phase tan-1realYtotalimgYtotal (blue) along with measured phase (using lock in amplifier) (red) to determine fir parameters........... 24

Figure 12 Top Left A) spectrum showing the mechanical resonance of the tuning fork. The blue line denotes the frequency at resonance, (when measured with the tip away from the surface) while the red bar signifies the peak amplitude level. Top Right B) In an Idealess case where only elastic forces are present, the resonance frequency shift as illustrated. Bottom Left C) In an idealized case, there only viscus (damping) interactions are present, the amplitude at resonance decreases from its previous maximum. Bottom Right D) A typical viscoelastic interaction where the tuning fork experiences both a shift in resonance frequency, and a decrease in driving amplitude....................................................... 27

Figure 13 Left) schematic displaying how the frequency modulation scheme is implemented within our microscope. Right Above) Screen capture from the Nononis tuning fork driving software, showing a spectrum of tuning fork amplitude as a function of frequency. Right Below) Screen capture from the Nanonis tuning fork driving software showing the phase of the tuning forks current response (measured with respect to the driving potential) as a function of frequency. 29

Figure 14 a) Left: Tuning forks total current spectral response (red trace) and its calculated (using expression 2.8-1) spectral response when the parallel capacitance is factored out to obtain the current response from the RLC portion, the latter being proportional to the tuning fork's mechanical displacement. b) Right: comparison of the WGAS acoustic transducers response, versus the calculated tuning forks mechanical displacement. ....................... 32

Figure 15 Left) Diagram showing the fixture and components which holds the tuning fork. The top surface of the fixture is where the acoustic sensor is placed. Also pictured are the 3 threaded rods and brass thumb screws which tighten against the acrylic plate that are used clamp the WGAS sensor to the fixture (green scale bar $\sim 6.5 \mathrm{~cm}$ ). Middle) Exploded diagram specifically showing a) fixture, b) collet, c) Macor and tuning fork, d) collet clamp. Right) A false color image is overlaid on top of this surface indicating the location of largest sensitivity in orange and yellow, whereas the location of least sensitivity appears in dark blue (green scale bar $\sim 3.7 \mathrm{~cm}$ ). 34

Figure 16 Sensitivity to different ways of mounting the QTF. Left: Tuning fork electrical response at a variety of collet nut (piece ' $d$ ' in Fig. 14)angular displacements including associated quality factors. Right: WGAS spectral response at a variety of collet nut angular displacements. 36

Figure 17 Above: A spectrum showing the SANM response when a driven tuning fork is swept through resonance with the tip placed a few microns above the sample (which is placed in intimate mechanical contact with the sensor). 37 
Figure 18: Characterizing the acoustic sensors directly. Note graph displays power relative to the resonance frequency, with domain extending between $22.25 \mathrm{k} \mathrm{Hz}$ and $42.55 \mathrm{k} \mathrm{Hz}$.

Figure 19 Block diagram showing the elements involved in measuring FPGA noise. The signal emanates with the user at the personal computer, which is connected to the FPGA card via the PCIE interface. The analog signal is then sent from the FPGA board, down the length of cable ( $5 \mathrm{~m}$ here), before reaching the breakout box. The final signal is measured at the Breakout boards BNC output terminals using a digital storage oscilloscope........ 41

Figure 20 Left) Osciloscope measurment of the FPGA ADC noise level at 50mV offset and $5 \mathrm{~m}$ long cable. Right) Osciloscope measurment of the FPGA ADC noise level at 50mV offset and $1 \mathrm{~m}$ long cable. 41

Figure 21 Sallen-Key architecture. 44

Figure 22 Left) A bode plot of the second order Sallen-key Butterworth low pass filter. Plotted in red is the filters theoretical frequency response with cutoff frequency of $10 \mathrm{~Hz}$. Plotted in blue is the measured frequency response with $-3 \mathrm{~dB}$ point determined to be $8.9 \mathrm{~Hz}$. Right) A bode plot of the second order Sallen-key Butterworth low pass filter. Plotted in red is the filters theoretical frequency response with cutoff frequency of $2.5 \mathrm{KHz}$. Plotted in blue is the measured frequency response with $-3 \mathrm{~dB}$ point determined to be $2.44 \mathrm{KHz} .45$

Figure 23 Left) The noise level at the circuit output, after the second order Sallen-key low pass filter (channel A), and summing amplifier, with $50 \mathrm{mV}$ applied to the input of channel A, and channel B grounded. Right) The noise level at the circuit output, after the second order Sallen-key low pass filter (channel B), and summing amplifier, with 1V applied to the input of channel B, and channel A grounded. 46

Figure 24 Top) The constructed Filter circuit board with SIO components inside a metal shielded case. The positive and negative regulators outlined in red, the two second order Sallen-Key filters in yellow, and the summing amplifier outlined in green (green scale bar $\sim 4 \mathrm{~cm}$ ). Bottom The schematic circuit diagram illustrating the positive and negative regulators outlined in red, the two second order Sallen-Key filters in yellow, and the summing amplifier outlined in green.

Figure 25 Top Leftt) Power spectral density plot of the FPGA noise before filter. Top Right) QQ plot comparing the FPGA noise distribution against a standardised gaussian distribution. Bottom Left) Power spectral density plot of Filtered drive signal. Bottom Right) QQ plot of filtered drive signal compared to stardardised gaussian distribution. . 48

Figure 26 Left) The constructed SIO differential amplifier circuit constructed on protoboard, placed inside a recycled RF can, (green scale bar $\sim 4 \mathrm{~cm}$ ). Right) Schematic diagram displaying the simplified low differential amplifier and low pass filter circuitry in red, with bipolar voltage regulation in red. Note the figure is displayed in a block diagram for clarity. The $12 \mathrm{~V}$ output from the voltage regulators in red are physically connected to the $12 \mathrm{~V}$ input to the op amp outlined in green. 50

Figure 27 Left) Rendering of Acrylic humidity chamber, designed in solid Works and manufactured using a laser cutter and Dichloromethane cement. Chamber can easily 
maintain relative humilities in excess of $80 \%$, (green scale bear $\sim 10 \mathrm{~cm}$ ). Right) A) External air compressor, B) Pressure regulator, C) Ball valve, D) Tubing to direct humid air, E) Rubber stopper, F) Erlenmeyer flask, G) Water, H) Open cell foam diffuser, I) Humidity chamber. 52

Figure 28 Left) Exploded diagram displaying the specially designed drive coupler design linking the stepper motor and threaded positioner shaft. The two brass cylinders' nest inside each other with keyed slots aligning to allow compliance in the axial direction, (green scale bar $\sim 8 \mathrm{~cm}$ ). Right) Stepper motor controller including optical encoder knob to manipulate shaft rotation, 3 switches (with corresponding LED indicators) to select the desired arrangement of stepper motors, and switch (below safety) to toggle between high speed and fine stepping, (green scale bar $\sim 6 \mathrm{~cm}$ ). 53

Figure 29 A schematic showing the electrical connection present in the tunneling current circuitry (measuring DC current contributions here) The probe can either be biased at the tuning fork by adding a DC potential to the ADD input of the OC4, or directly at the current amplifiers input. 56

Figure 30 Left) Spectrum taken while characterizing the tuning fork for an experiment on 2018-03-15. Notice SANM peaks near $31.3 \mathrm{kHz}$, while QTF, and WGAS peak between 31.5-31.6 kHz. Right) Spectrum taken while characterizing the tuning fork for experiment on 2018-04-06. Notice SANM again peaks near 31.3K, while QTF and WGAS peak slightly above. 58

Figure 31 Left) Signal trace captured in the time domain using a Tektronix digital storage oscilloscope and computer interface showing induced noise in an antenna probe placed near the Cathode Ray Tube (CRT) display on the SR850 lock in. Right) Power spectral density plot of the same CRT noise displayed left. Note the second harmonic peak is located between $31.0 \mathrm{kHz}$ and $31.4 \mathrm{kHz}$. 59

Figure 32 a) SEM imaged captured on 2018-02-08 of QTF probe (ADC001) showing probe morphology before compliance experiment. b) SEM image captured on 20118-02-08 of QTF probe (ADC001) after the amplitude QTF amplitude as decreased from $100 \%$ to $90 \%$, showing no deformation to the probes apex. c) SEM imaged captured on 2018-02-09 of QTF probe (ADC001) showing probe morphology after the amplitude decreased from $100 \%$ to $80 \%$ and showing plastic deformation to the probes apex. The experiments were performed in frequency modulation modality.

Figure 33 a) SEM imaged captured 2018-06-13 of QTF probe (ADC027) showing probe morphology before compliance experiment. b) SEM imaged captured 2018-06-14 of QTF probe (ADC027) showing probe morphology after the QTF's amplitude decreased from $100 \%$ to roughly $50 \%$; experiment performed under frequency modulation control.c) SEM imaged captured 2018-06-15 of QTF probe (ADC027) showing probe morphology after the QTF's amplitude decreased from 100\% to roughly $25 \%$; experiment performed under constant frequency driving voltage. 70

Figure 34 Probe: etched gold (ADC001), Sample: Hydrophilic Si, Humidity: 56\%, original data acquired at $0.4 \mathrm{~ms}$ time constant and $4 \mathrm{~ms}$ data rate. Left) Graph displaying the QTF's 
mechanical amplitude as a function of distance for the experiment on 2018-02-09 that resulted in the visible deformation of the probes apex seen in figure $30 \mathrm{c}$ ). Right) Plot of the interaction damping coefficient as a function of distance for the experiment on 201802-09 that resulted in the visible deformation of the probes apex seen in figure 30c). Fluctuations in the plot of interaction damping coefficient is perceived larger than the decrease in mechanical amplitude because the amplitude plotted relative to its absolute zero. 72

Figure 35 Probe: etched gold (ADC001). Sample: Hydrophilic Si. Humidity: 63.8\%, original data acquired at $0.4 \mathrm{~ms}$ time constant and $4 \mathrm{~ms}$ data rate. Left) Graph displaying the QTF's mechanical amplitude as a function of distance for the experiment on 2018-0209 that resulted in no visible deformation of the probes apex seen in figure 31b). Right) Plot of the interaction damping coefficient as a function of distance for the experiment on 2018-02-09 that resulted in the no visible deformation of the probes apex seen in figure $31 b)$. 72

Figure 36 Approach retraction curve between new etched gold probe (ADC029) and hydrophobic HF etched Si sample, humidity: $69.4 \%$, Left) Plot of WGAS signal as a function of distance for the QTF probe in FM. Right) SANM signal in red displaying the raw data as a function of distance, and black displaying a dataset containing a smoothed SANM signal as a function of distance. The vertical lines help noticing that the acoustic and WGAS signals start to significantly change at a different probe-sample distance. .. 76

Figure 37 Left) probe identified as ADC029 before the experiment. Right) Probe identified as ADC029 after the experiment. Note possibly slight plastic deformation in the probes apex as well as possible mass transfer. 77

Figure 38 Approach retraction curve between new etched gold probe (ADC029) and hydrophobic HF etched Si sample, Humidity: 69.4\%. Left) dataset containing a smoothed SANM signal against the inverse of the WGAS signal, showing poor correlation between the two signals. The source of acoustic emission is not of damping nature then. Right) dataset containing a smoothed SANM signal against the resonant frequency shift showing better correlation. This is in agreement with the consideration that the acoustic emission (WGAS signal) is an elastic energy-dissipation channel (reflected in the frequency shift) of the probe-fluid-substrate interaction. 78

Figure 39 Left) SEM image of probe identified as ADC029, used on hydrophobic sample (HF etched $\mathrm{Si}$ ) to generate the plots displayed in figure 38 (left above, and left below). Right) SEM image of probe identified as ADC025, used on hydrophilic sample (Piranha cleaned $\mathrm{Si}$ ) to generate plots displayed in figure 38 (right above, and right below). ....... 80

Figure 40 Upper left) Plot of WGAS approach curve using probe identified as ADC029 on hydrophobic sample (HF etched $\mathrm{Si}$ ). Upper right) Plot of WGAS retraction curve using probe identified as ADC029 on hydrophobic sample (HF etched Si). Lower left) Plot of WGAS approach curve using probe identified as ADC025 on hydrophilic sample (piranha cleaned $\mathrm{Si}$ ). Lower right) Plot of WGAS retraction curve using probe identified as ADC025 on hydrophilic sample (piranha cleaned Si). In all cases lock-in time constant: 10 $\mathrm{ms}$, data rate:50 $\mathrm{ms}$. 82 
Figure 41 Approach retraction curves using an etched gold probe and HOPG sample at $63 \%$ humidity. WGAS time constant: $10 \mathrm{~ms}$, Data rate: $50 \mathrm{~ms}$. Left) a the 1 st interaction Right) $12^{\text {th }}$ interaction 84

Figure 42 SEM micrograph of an early AFM probe. Left) the initial characterization after probe fabrication, Right) subsequent characterization after interacting with sample. .... 85

Figure 43 Approach retraction curve using AFM probe and Poly(2-ethyl-2-oxazoline) sample, Humidity is unknown. a) A plot of the WGAS sensor as a function of distance. This trace from the approach retraction curve using the AFM tip. The magnitude of the trace has been normalized such that a magnitude of 1 refers to the probes amplitude away from the surface. B) A plot of the AFM probes resonant frequency shift as a function of distance. C) A plot of the SANM sensor as a function of distance. Here the sensors magnitude has been normalized such that 1 refers to the sensors acoustic reading while the probe and sample are not interacting. 88 


\section{List of Abbreviations}

$\mathrm{AC}$

AFM

BVD

CRT

DC

DFT

ENBW

EOM

FPGA

$\mathrm{HF}$

HOPG

MCL

NSOM

PID

PSD

QQ

QTF

RF

$\mathrm{RH}$

RLC

SANM

SEM

SHO

SNAM

SNR

SPM

STM

UHV

WGAS
Alternating Current

Atomic Force Microscope

Butterworth-Van Dyke

Cathode Ray Tube

Direct Current

Discrete Fourier Transform

Effective Noise Bandwidth

Equation of Motion

Field Programmable Gate Array

Hydrofluoric acid

Highly Ordered Pyrolytic Graphite

Mad City Labs

Near- Field Scanning Optical Microscopy

Proportional-Integral-Derivative

Photon Sensitive Detector

Quantile-Quantile

Quartz Tuning Fork

Radio Frequency

Relative Humidity

Resistor-Inductor-Capacitor

Shear-Force Acoustic Near-Field Microscope

Scanning Electron Microscope

Simple Harmonic Oscillator

Scanning Near-Field Acoustic Microscopy

Signal to Noise Ratio

Scanning Probe Microscope

Scanning Tunneling Microscope

Ultra-High Vacuum

Whispering Gallery Acoustic Sensor 


\section{Chapter 1}

\section{$\underline{\text { Introduction }}$}

\section{(1.1) Introduction and Background on Proximal Probe Microscopy}

This thesis discusses developments to the Sheer-force Acoustic Near-field Microscope (SANM), and its contributions to surface metrology. For clarity, it will be helpful to contextualize these new developments within the larger field of scanning probe microscopy (SPM). This will be done by first discussing a brief historical progression of the research by highlighting some noteworthy developments in SPM that have made this work possible. SPM is discussed in many periodicals including Wiesendanger's book "Scanning Probe Microscopy and Spectroscopy" [6], which is an invaluable resource.

In 1969 Tabor and Winterton directly measured normal and retarded Van der Waals forces [7]. These Van der Waal forces are often the predominant interaction mechanism in systems of electrically neutral atoms or molecules, which result in part due to "finite fluctuating dipole and higher multipole moments at very short time intervals" and dominate at small separation distances [6](pg. 211). These measurements by Tabor and Winterton were performed using a surface force apparatus consisting of two cylindrically curved mica sheets oriented such that the convex surfaces faced each other (with symmetry axis rotated 90 degrees, to minimize the interaction surface area). One sheet is held stationary and attached to a cantilevered spring, while the other sheet's vertical position may be manipulated. As the two mica sheets are brought near each other, their displacement, and eventual 'flick' to contacting each other (due to van der Waals interaction forces), is 
monitored using optical interferometry with an accuracy of 3 Angstroms [7]. Knowledge of the cantilevers spring constant and displacement allows measurement of the interaction forces. It was found that separation distances below $10 \mathrm{~nm}$ exhibit normal Van der Walls forces $\left(F_{V D W}\right)$ (exhibiting the characteristics $F_{V D W} \sim-s^{-7}$, where $\mathrm{s}$ is the separation distance), while separation distances greater than $30 \mathrm{~nm}$ exhibit 'retarded' Van der Waals forces $\left(F_{V D W} \sim-s^{-8}\right.$, where again $\mathrm{s}$ is the separation distance) [7]. This surface force apparatus was an important tool for measuring precise interaction forces as a function of distance. However, the tools lack of lateral resolution limited its use as a general microscopy tool [6].

Surface profilometers, in contrast, do allow limited topographical characterization of samples. In 1982 Teague et al utilized a 3-dimensional stylus profilometer to characterize surface roughness of nonconductive samples [8]. This was done by placing a stylus probe in direct contact with the surface and raster scanning over the sample whilst maintaining a 'set' interaction force between the sample and stylus [8]. However, the required stylus-sample interaction force was relatively large necessitating a similarly large stylus apex radius on the order of $1 \mathrm{um}$ to minimize damage to samples which resulted in poor lateral resolution [6].

In the early 1980 's, innovations in quantum tunneling experiments advanced the field of probing microscopy. In a 1982 Applied Physics Letters publication, Binnig, Rohrer, Gerber, and Weibel reported on the first successful tunneling through a controllable vacuum gap between two electrodes (one a tungsten tip, and the other a platinum surface). In this study, they showed that the tunneling current is related to the 
separation distance between electrodes as expected [9]. Later that year, Binnig and Rohrer published a paper in the journal Surface Science where they reported development of a successful Scanning Tunneling Microscope (STM). In this article, they included surface reconstructions of several samples including a $7 \times 7$ unit cell $\mathrm{Si}$ (111) displaying atomic structure [10]. These image reconstructions were obtained by scanning over the conductive sample and varying the probes height above the surface (and therefore spacing between electrodes) to maintain a constant tunneling current. In this way, a reconstruction is created in which the topography is related to the voltage applied to the extending piezo electrode to maintain a constant probe-sample distance (hence constant tunneling current) during the scanning process $[10]$.

This tunneling method was adapted to non-conductive surfaces with the advent of the scanning force microscope, better known as Atomic Force Microscope (AFM) [6]. This design first used a sharp diamond tip probe to interact with the sample affixed to a cantilever formed out of gold foil. While scanning across a sample, interaction forces between the diamond tip probe and sample cause the probe and cantilever to deflect in the normal direction. To accurately measure the cantilevers deflection, a tunneling junction was created between the gold foil cantilever and a second STM probe positioned above the first cantilever [11]. With this experimental setup, many operational modalities were implemented. In principle, interaction forces between the diamond tip probe and sample cause deflections in the cantilever (related to its effective spring constant), which in turn modulate the tunneling gap dimension. This modulation results in tunneling current fluctuations which can be related to the interaction force applied to the cantilever [11]. In 
1988, Meyers and Armer simplified the AFM design by implementing an optical detection scheme instead of tunneling current. In this design, a Helium neon laser is reflected off the back of a tungsten stylus cantilever (containing a micro mirror), and detected via a Photon Sensitive Detector (PSD) [12]. Like before, probe-sample interactions manifest as small deflections in the cantilever. Now, however, instead of this modulating a tunneling gaps spacing, the cantilevers deflection deviates the lasers optical path, which is detected via the PSD [12]. A similar optical detection scheme is still currently used in many modern AFM's.

In addition to measuring Van der Waal's forces acting in the normal direction, a better understanding of atomic friction, or lateral force interactions, was desired. These measurements were first accomplished by Mate et al in 1987 by utilizing an AFM and optical interferometry [6]. Changes in intensity (corresponding to friction interactions) were the result of path differences between one beam of light reflected off a stationary optical flat, and a second beam reflected off the side of a compliant tungsten stylus cantilever (which deflected parallel to the surface under the influence of friction) [13]. With a small load applied to the cantilever, a graphite sample was scanned under the tip "displaying features with atomic periodicity of the surface" [13]. Lateral (or shear) force microcopy would later become an important tool in Near-Field Scanning Optical Microscopy (NSOM) for controlling the probe-sample distance, where it was implemented in an AC-modality (instead of the Direct Current (DC) reported above).

NSOM exploits the high special frequencies present in evanescent waves which are localized within the optical diffraction limit $\left(\frac{\lambda}{2}\right)$ of the samples surface. By scanning an 
optical probe that comprises a nm sized metallic aperture near a samples surface, the diffracted light can be extracted in the far field to create an intensity map with high spatial resolution [6]. To accomplish this, the metallic aperture must be positioned within $10 \mathrm{~nm}$ of the sample as the intensity of the evanescent waves decreases quadratically away from the sample [6]. In 1992 Betzig et al solved this problem by utilizing an etched single mode optical fiber with aluminum coated aperture attached to a piezo ditherer as both an NSOM optical probe and as a shear force sensor [14]. The NSOM fiber probe was laterally excited via the ditherer at its resonant frequency; the shear force interactions would shift the fibers resonant frequency, causing its driving amplitude to decrease. This was able to be detected via the Alternating Current (AC) component of the transmitted optical signal [14]. Because the shear interaction forces are related to the probe's height above the surface, the probe's oscillation amplitude was used as a feedback control mechanism to drive the samples piezo positioners, and maintain a constant probe-sample separation distance [14].

\section{(1.2) The Adaption of Tuning Fork Sensors in Probe Microscopy}

In 1995, Karri and Grober published a formative paper wherein they utilized a piezo electric quartz tuning fork (QTF) to electrically detect shear interaction forces (whose source at the time was not well known) between the NSOM probe and sample. They then used this sheer force as a control signal to reliably position the probes apex within $25 \mathrm{~nm}$ of the samples surface [15]. This was accomplished using a single mode optical fiber etched to a taper with an aluminum aperture fabricated at its apex. The fiber was glued to one leg of a QTF, ensuring the long axis of both the fiver and tuning fork were aligned in the same 
direction [15]. The tuning fork and probe were then excited using a piezo ditherer (at the high $\mathrm{Q}$ mechanical resonance of the tuning fork) such that the probes apex oscillates laterally above the samples surface [15]. Given the piezo electric nature of quartz, the oscillations of the tuning forks tines generate a current-signal, which can be measured using synchronous lock-in detection. As the probe interacts with the sample, via a shear-force, the QTF's resonant frequency shifts, and the probes amplitude decreases [15]. Both parameters can be implemented as positioning control signals due to their dependence on separation distance.

\section{(1.3) Sheer Force Acoustic Near-Field Microscopy}

Apart from NSOM, another probing technique of interest is Scanning Near-Field Acoustic Microscopy (SNAM), developed by Gunther et al in 1989. Unlike AFM, which primarily measures van der Waal interaction's or the lateral shear force, SANM claims to offer sensitivity to measure the force acting on the 'coupling gas' localized between the edge of a quartz tuning fork (used as an acoustic transducer) and the sample [16]. The force supplied by the coupling gas on the QTF acoustic transducer causes a decrease in both its mechanical resonance frequency and amplitude, which was used as a control signal for topographical positioning in the usual way. It was shown that changes in the atmospheric pressure (causing a change in the mean free path of the gas molecules) did have a prominent effect on frequency shift and amplitude for a given probe sample separation distance [16]. A very different acoustic monitoring scheme is used in the current system where acoustic 
transducers are used to detect both the mechanical osculation of the tuning fork tines, as well as acoustic emission from the adsorbed mesoscopic fluid.

\section{(1.4) Interfacial Shear Force Microscopy}

In 2000, Karrai and Tiemann published a paper in Physical Review B titled 'Interfacial Sheer Force Microscopy'. Unlike previous papers where the viscus sheer interaction force is studied as a parameter controlling the vertical position of an NSOM probe above the surface, Karrai et al investigated the source of the viscus sheer interaction. They presented a method to explicitly quantify the "viscus friction and sheer elastic stress" as a function of probe sample spacing [17]. The experimental setup in the 2000 paper was

like the 1995 paper, however the (now unused) NSOM optical fiber probe was replaced with short electromechanically etched gold wire glued to the QTF's leg. The paper discussed the viscus and elastic interaction forces that are present when the tapered gold probe approaches the sample of either cleaved graphite or $n$ doped GaAs. They concluded that "even under vacuum conditions $\left(8 \times 10^{-7}\right.$ mbar or $6 \times 10^{-7}$ Torr $)$, the measured viscous and elastic shear stress (i.e., velocity dependent) are directly attributable to a third body filling the tip-sample gap" [17].

The investigation of this third body's properties (assumed here in the form of an adsorbed mesoscopic viscoelastic fluid layer) will be the primary focus of this thesis. This investigation will include analysis of the third body's viscoelastic properties using $i$ ) Shearforce Near-field Acoustic Microscopy [18] (that uses a piezoelectric tuning for driving a tapered probe and a transducer to monitor the acoustic emission of the fluid trapped, under 
shear, between the probes apex and the substrate), and ii) a Whispering Gallery Acoustic Sensor (WGAS) $[19,19]$ to directly monitor (via acoustic means) the amplitude of the QTF's mechanical oscillations. Through this analysis, we aim at better assessing samples properties such as adhesion [1] [2], wetting, [3] the nature of the hydrophobic interaction (which is relevant in many areas of study such a protein folding [4]), as well as interfacial friction which has wide ranging applications including desalination [5]. Additionally, acoustic emission from the adsorbed mesoscopic fluid in confinement will be studied using the Shear-Fore acoustic Nano Metrology sensor positioned below the sample. 


\section{Chapter 2}

\section{Experimental Setup}

\section{Exploded Diagram of the Shear-Force Acoustic Near-Field Microscope}

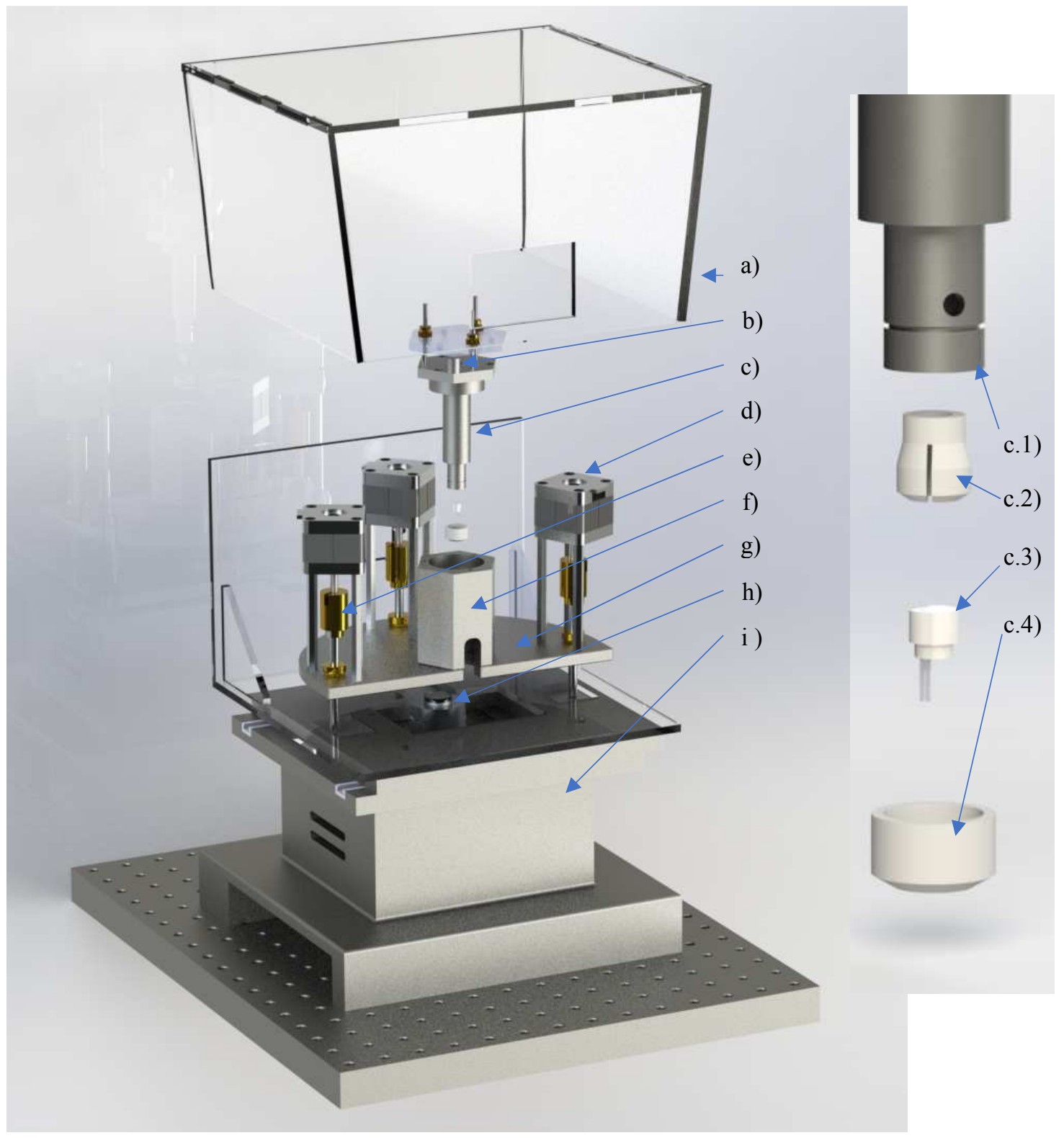

Figure 1 Partially exploded diagram of the individual components that constitute the microscope used in this thesis. a) Humidity chamber, b) WGAS acoustic sensor, c) tuning fork fixture assembly, c.1) tuning fork fixture, c.2) collet chuck, c.3) Macor with tuning fork installed, c.4) collet clamp, d) stepper motor, e) stepper motor drive coupler assembly, f) whispering gallery acoustic cavity, g) SPM base plate, h) SANM acoustic sensor, i) microscope base incorporating piezoelectric positioners. 


\section{(2.1) The Tuning Fork Based Sensor}

The Quartz tuning fork oscillator plays a central role within the Shear-Force Acoustic Near-Field Microscope. Fabrication of the tuning fork based sheer force sensor begins by preparing the Quartz oscillator. QTF's have become a fundamental electronic building block, and are utilized in devices requiring precision frequency control such as common clocks and wrist watches [20]. As such, they are readily available from online electronics parts retailers. The tuning forks used in this thesis are produced by the ECS Inc. international company, part number ECS-3x8x. For protection and stability, the crystal oscillator is sold in a packaged form inside a metallic 'can' enclosure (see figure 2a); four our applications the latter needs to be removed. We typically follow one of two methods: i) One method involves side cutter plyers. This is done by holding the tuning fork in one hand (with the electrical leads pointing upward), while the other hand manipulates the plyers applying slight pressure (with the blades) against the metallic can, (right at the point where the hard epoxy seals the top of the can). Slowly pressing the blades around the can, this method gradually fractures the epoxy until it turns to dust and the tuning fork cleanly slides out of the can (figure $2 \mathrm{~b}$ ). This method is preferred as it yields only the tuning fork and leads without any artifacts of the can remaining, however it is more prone to fracturing the tuning fork. ii) A second method involves using a 'Can Opener' produced by Thor Labs with product number WR1. This device simply cuts away the end of the can revealing the tuning fork. However, the epoxy disc that is left behind reduces the usable length of electrical lead, and prevents the tuning fork from setting flush against a ceramic holder (figure 2c). (the holder is used to attach the QTF to the microscope frame while 
simultaneously electrically isolating it). Once the QTF is remove from its protective can, the electrical leads are threaded through the holes in the ceramic tuning fork holder, and epoxied in position (See figure 1 c.3).

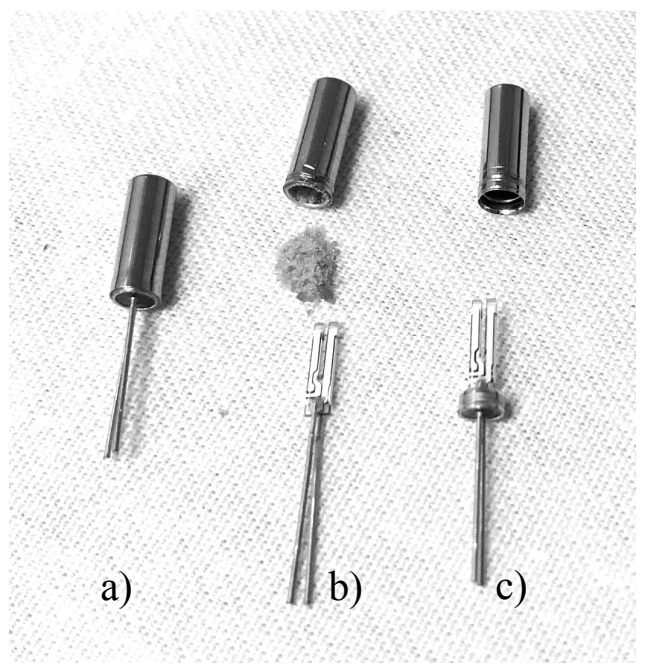

Figure 2 The QTF still in its protective 'can' housing is displayed in a). b) displays the tuning fork after its been removed from the can using the side cutter technique, note the epoxy has been crushed (visible above the TF) and no ring is present. c) displaying the can-opener technique where can is cut away, leaving the epoxy ring attached to the oscillator.

To create a complete sheer force sensor, the tuning fork must include a sharp probe to locally interact with a specific sample region. Sharply tapered probes can be fabricated though an etching process whereby a cylindrical wire or glass shaft is shaped into a tapered cone through a subtractive manufacturing process. This etching procedure falls into two categories; electrochemical etching of metallic wire, and conventional chemical etching for glass fibers. Each of these etch methods utilize the same physical property to create tapered conical probe tips. When the fiber or wire is submerged into an etching solution, a meniscus is formed via capillary action due to surface tension and adhesion to the shaft. The height that the meniscus travels up the shaft is dictated by [21] 


$$
h_{\text {meniscus }}=\left[2\left(\frac{\gamma}{\rho g}\right)(1-\cos \theta)\right]^{\frac{1}{2}}\left[1+\left(\frac{\gamma}{\rho g}\right)^{\frac{1}{2}} \frac{1}{r}\right]^{-\frac{1}{2}}
$$

Where $\gamma$ is the surface tension, $\rho$ the density of the etching fluid, and $\theta$ the contact angle with respect to the probe shaft. The etching processes consumes material contacting the etching solution in a direction that is normal to the probes surface. As material is consumed the probes radius becomes reduced thereby decreasing the meniscus height, generating a tip profile related to equation (1).

\section{(2.2) QTF Tapered Gold Probe Fabrication}

Probes are commonly manufactured using gold due to its low reactivity and high conductivity. This conductivity allows tunneling measurements to be conducted while simultaneously monitoring sheer interaction forces [17]. The gold probes presented here were fabricated using $125 \mu \mathrm{m}$ diameter, 3N5 gold wire (where 3N5 refers to the purity grade, or $99.95 \%$ pure). The fabrication process begins by attaching a $4-\mathrm{cm}$ long section of gold wire to an electrical (anode) contact, and placing the wire vertically on a $\mathrm{z}$ axis positioner with $5 \mathrm{~mm}$ of the wire protruding below the edge of the holder station. An etching solution consisting of one part $37 \%$ hydrochloric acid and one part $95 \%$ ethanol was then placed in a small beaker below the gold wire. A 3-cm long, 1/8" diameter, graphite rod was inserted into the solution and used as the cathode. During the etching process, Bubbles are created around the rod which present a problem. Allowing these bubbles to rise and break near the surface of the solution can agitate the meniscus leading to a detrimental effect on the probes surface roughness. One solution to prevent this agitation 
consists of trimming a plastic pipette into a cylindrical shape, and placing it concentrically

over the cathode. This cover streams the bubbles up the pipet walls where they are vented without disturbing etching (Fig, 3 left illustrates this etching setup).
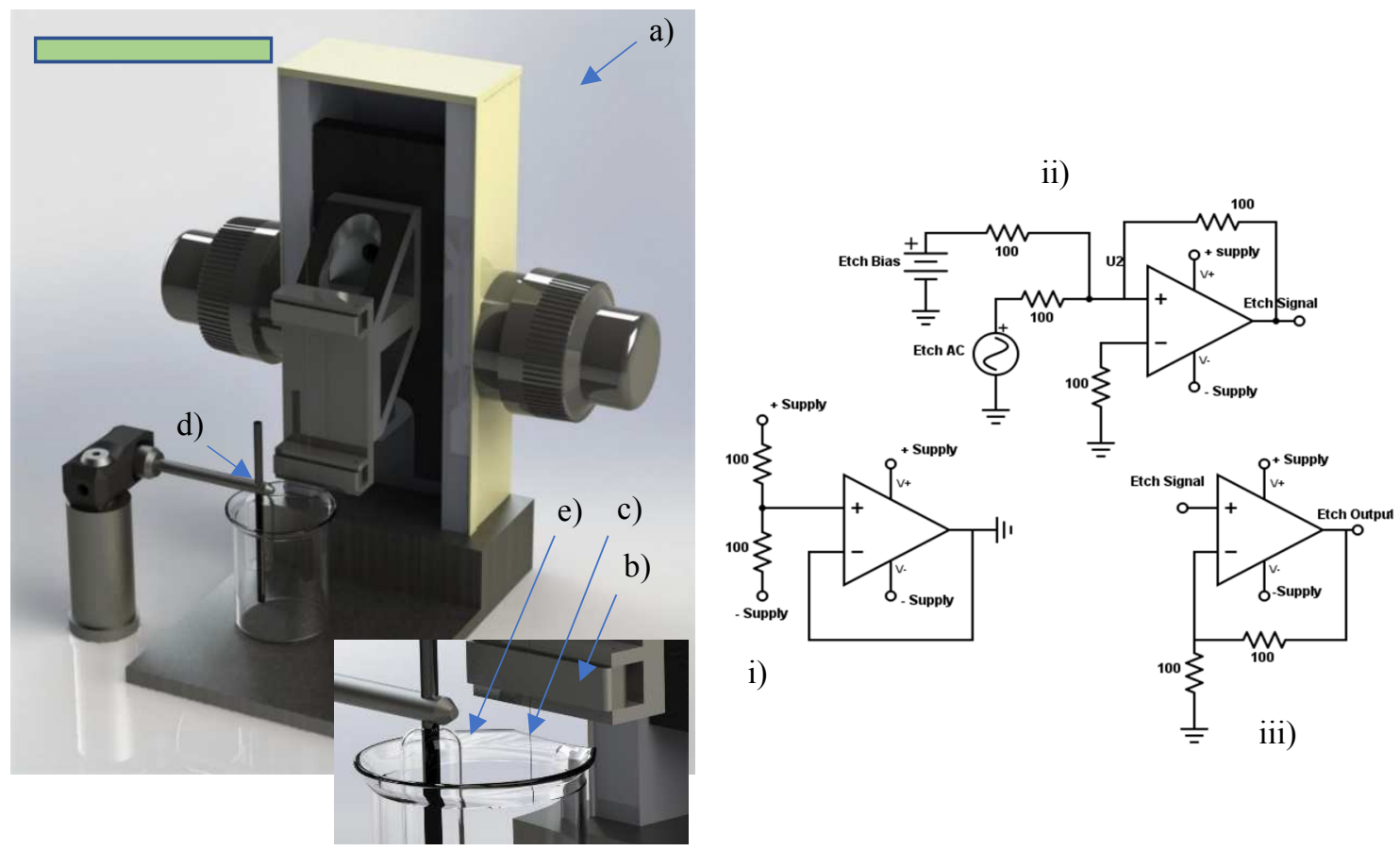

Figure 3 Probe fabrication via chemical etching. Left) Diagram showing the metallic tip etching setup with inset displaying close-up view of critical elements. a) z positioner, b) anode wire connection, c) gold etching wire, d) graphite cathode, holder and electrical connection, e) pipet bubble guide, (green scale bar $\sim 8.5 \mathrm{~cm}$ ). Right) Electrical schematic of etching power supply including unipolar to bipolar supply converter (with voltage follower to decouple the circuit impedance from the divider), Summing amplifier inside the Stanford Research Function Generator, and non-inverting amplifier with gain of 2. Note the schematic is presented in a simplified diagram. i) depicts a circuit used to convert a single ended supply into a bipolar supply for the op-amps, in turn creating a virtual ground that can be used by ii) and iii). ii) depicts an adding amplifier taking both the DC etch bias and the etch AC, and outputting a single signal at 'Etch Signal'. Finally, this 'Etch Signal' form ii) is fed into the 'Etch Signal' at the non-inverting input of iii) before being fed to the gold anode though the follower circuit at 'Etch Output'.

An electrical potential is then applied between the cathode and anode (to generate the etching current). This was accomplished using a Stanford research brand Function generator, with an additional output amplifier which was built (with a gain of two) to 
increase the maximum output potential, and decouple the function generators output impedance (Fig. 3 right). The final AC etching potential from this device consists of a 1 volt amplitude signal at $1500 \mathrm{~Hz}$, added to a 6 volts' $\mathrm{DC}$ bias. Then the $\mathrm{z}$ axis positioner holding the wire is lowered until approximately $2 \mathrm{~mm}$ of the gold wire is submerged, in the etching solution, and a $25-\mathrm{mA}$ peak current is measured.

As the etching process occurs, hydrogen gas is created at the graphite cathode, and chlorine gas is created at the gold wire anode. This Chlorine gas reacts with the gold electrode to produce gold chloride, which in turn can undergo a secondary reaction with the gold electrode. These reactions are listed below [22], where $E_{0}$ refers to the standard electrode potential.

$$
\begin{gathered}
\mathrm{Au}+2 \mathrm{Cl}^{-} \rightarrow \mathrm{AuCl}_{2}{ }^{-}+e^{-}, E_{0}=1.154 \mathrm{~V} \\
\mathrm{AuCl}_{2}{ }^{-}+2 \mathrm{Cl}^{-} \rightarrow \mathrm{AuCl}_{4}{ }^{-}+2 e^{-}, E_{0}=0.926 \mathrm{~V} \\
\mathrm{Au}+4 \mathrm{Cl}^{-} \rightarrow \mathrm{AuCl}_{4}{ }^{-}+3 e^{-}, E_{0}=1.002 \mathrm{~V}
\end{gathered}
$$

After etching for approximately 90 seconds, the current drops to zero. At this point, the $\mathrm{z}$ positioner is abruptly raised (by manually rotating the positioning knob), and the gold wire is removed and rinsed in an acetone bath. Finally, the etched portion is cut from the remaining gold wire yielding a tapered probe approximately $700 \mu \mathrm{m}$ in length. After characterizing the morphology with a Hitachi S-4160 scanning electron microscope (see figure 4, left), the etched gold tips are ready to be attached to a quartz tuning fork sensor. The probes are affixed to the tuning fork using a 2-part 3M epoxy (3M DP420). Upon preparing a small quantity of epoxy, a needle can be used to transfer a drop to the end of one tuning fork tine. Care should be taken to deposit the smallest amount of glue needed, 
as excess epoxy will decrease the quality factor of the tuning fork probe (the same is true for etched probes with excessively long tails).
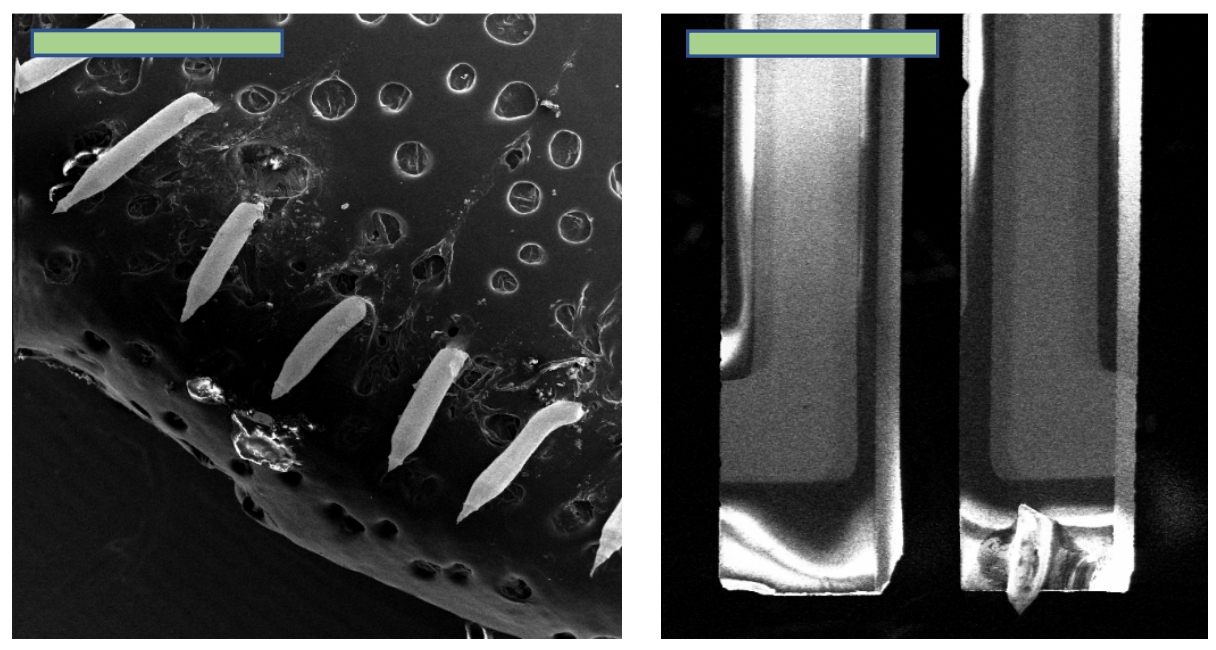

Figure 4 Left) SEM image of gold tips setting on carbon tape after being etched and cleaved from the wire, (green scale bar $=1.25 \mathrm{~mm}$ ). Right) SEM image of a gold probe after being epoxied to a tuning fork (green scale bar $=650 \mathrm{um})$.

The tapered probes are large enough that they can be manipulated using a pair of sharp tweezers, and positioned atop the epoxy such that the tip protrudes a few microns beyond the end of the tine (see figure 4, right). To better understand how the probe's quality factor is effected when probes are attached to the QTF oscillator, a simple experiment was implemented wherein small masses were incrementally added to one arm of the tuning fork to test its response. First, the quality factor of a bare tuning fork (with no additional mass) was assessed as a control. Subsequently, a small sections of gold wire (used as mass) was epoxied to the tuning fork, and a spectrum was recorded once the epoxy had fully cured. This process was repeated 2 more times with additional masse to produce the frequency response curves seen in figure 5 left. It was found that indeed, the more mass added to a tuning fork tine, the lower a probe's quality factor became. 

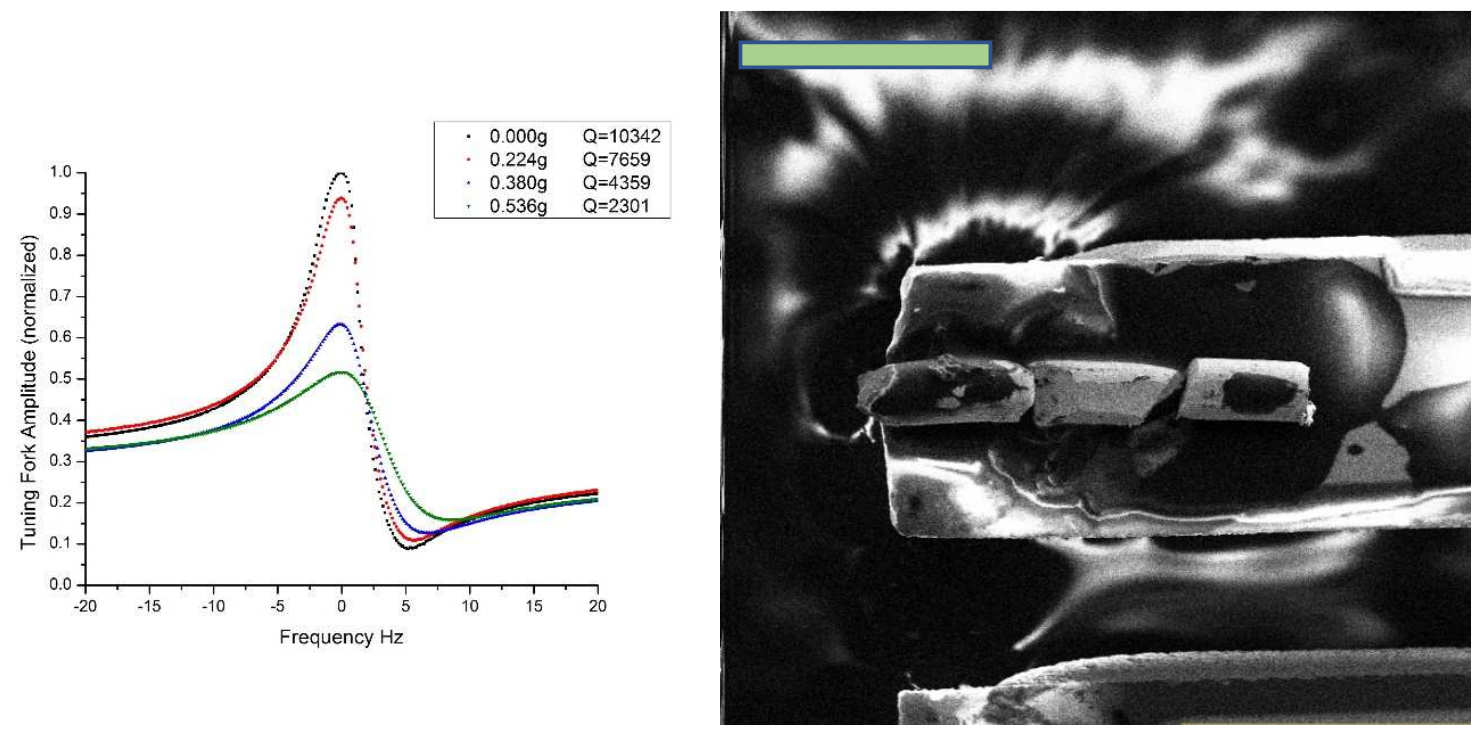

Figure $5 \boldsymbol{L e f t}$ ) Tuning fork spectral response and quality factor corresponding to different added mass. Right) SEM image of the tuning fork after the addition of the $3^{\text {rd }}$ mass (green scale bar $=450 \mathrm{um}$ ).

\section{(2.3) QTF/AFM Probe Fabrication}

Probes composed of an AFM cantilever attached to a tuning fork were also used in this project. One advantage of these probes, is that the tip geometry is relatively standardized due to automated factory production. This standardization allows easier comparisons to be made when analyzing results taken with two different probes. It is no longer necessary to consider variations in probe length and tip radius caused by etching. Another advantage is that the AFM probes are composed of silicon which is more robust than gold. This robustness becomes important when considering probe deformation and compliance. There are difficulties however, when implementing AFM/QTF sensor probes. The small size of the cantilever makes it difficult to manipulate and glue the probe in the desired position on the tuning fork. (However, this small size can be a positive aspect, as the reduced mass increases the quality factor of AFM/QTF probes). A second disadvantage 
is that the cantilever is glued under the tuning fork, and directly to the insulating quartz. This position prevents electrical connection with a conductive trace, making tunneling measurements difficult. One solution comprises creating a mask and sputtering a conductive layer to connect the cantilever to the electrical tuning fork trace, however it is very labor intensive and has yielded mixed results.

The QTF/AFM probes were fabricated using a modified 5-axis positioner (Thor Labs, MBt616D, see fig. 6) to ensure the cantilever were place in the desired location atop the tuning fork (with cantilever parallel to the quartz surface, as shown in fig. 7) First the un-caned QTF is epoxied into a ceramic (holder as described above), and then attached to the AFM probe mounting jig with tines facing upward. This jig is constructed from a semicircular stainless steel plate with the TF mounted at the center of the arc, and 3 threaded micro positioning legs positioned at 12,3, and 9 o'clock (as shown in figure 6a). This plate provides variable adjustment in both tilt directions, and thus allows the tuning fork to be aligned such that the surface atop the tuning fork tine is parallel to the surface of the base plate. This can be verified using a stereo optical microscope positioned overhead. Tweezers are then used to place the stub portion of the AFM probe (upside down) on adhesive tape located at the end of a mounting arm (attached to the Thor Labs positioner, see figure 6 c) such that the cantilever and tip are protruding over the edge. 


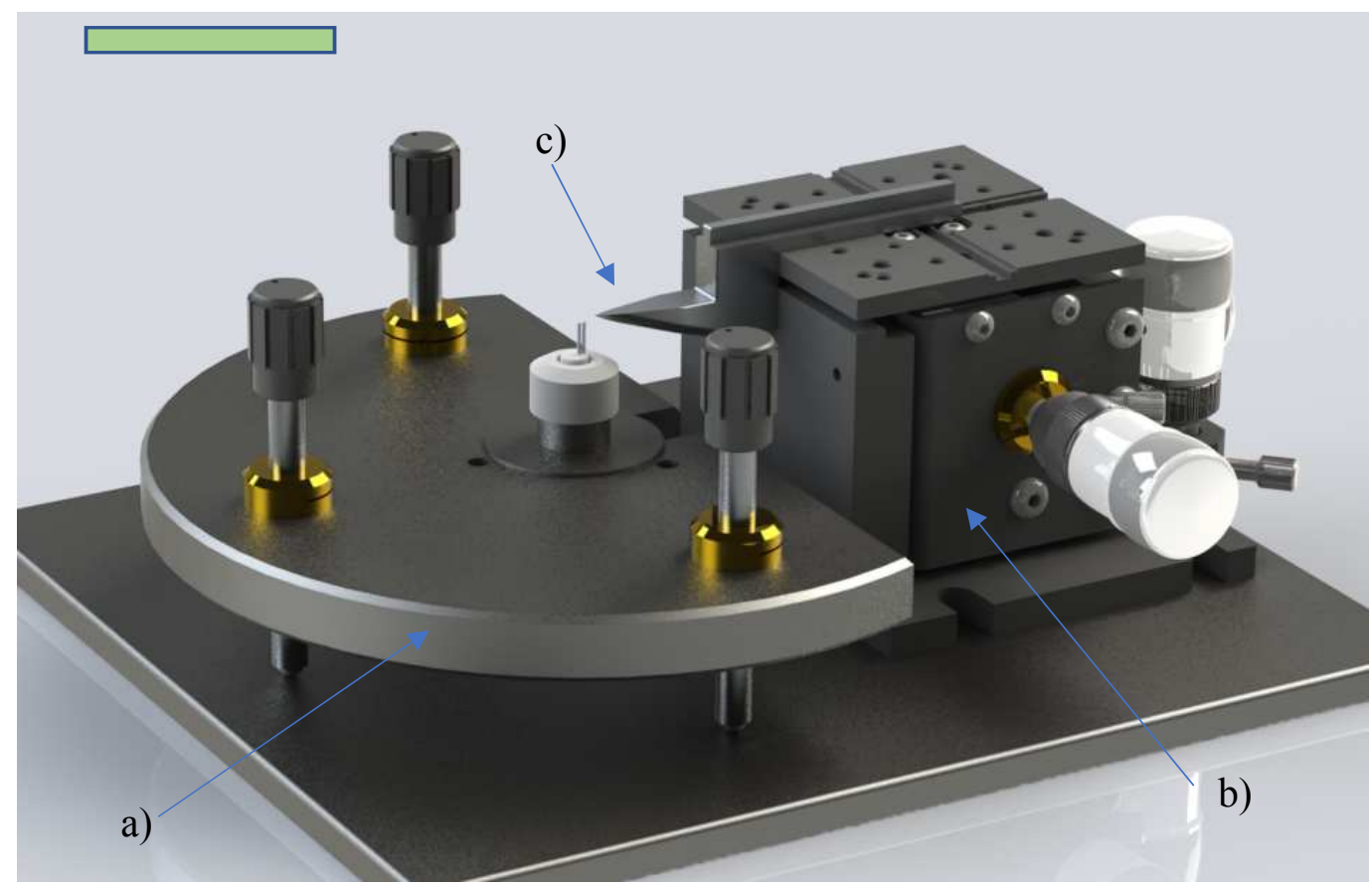

Figure 6 Diagram of the 5-axis apparatus used to mount AFM cantilevers to the bottom of tuning fork, (green scale bar $\sim 4 \mathrm{~cm}$ )). Apparatus composed of a) jig, b) Thor Labs 3 axis positioner, c) mounting arm. (Note, the Thor Labs 3 axis positioner component modeled in this caption is from Thor Labs product guide)

This two-piece design allows the Thor Labs positioner (containing the AFM probe) to be picked up and set aside, providing plenty of space to deposit a small drop of glue on the tuning fork without the cantilever nearby. With the glue on the tuning fork, the Thor labs positioner is then replaced, ensuring the mounting arm with cantilever is positioned a few microns above the quartz, in the desired orientation. The positioner carrying the AFM tip is slowly lowered until the cantilever just contacts the epoxy and quartz surface. It's possible to allow the cantilever to dry in this position, however better results are usually attained by lowering the positioner further until the cantilever sheers off from the stub (still attached to the mounting arm). In this way, the AFM tip can settle through the viscous 
epoxy to make firm contact with the quartz, thereby eliminating the possibility of the probe drifting.

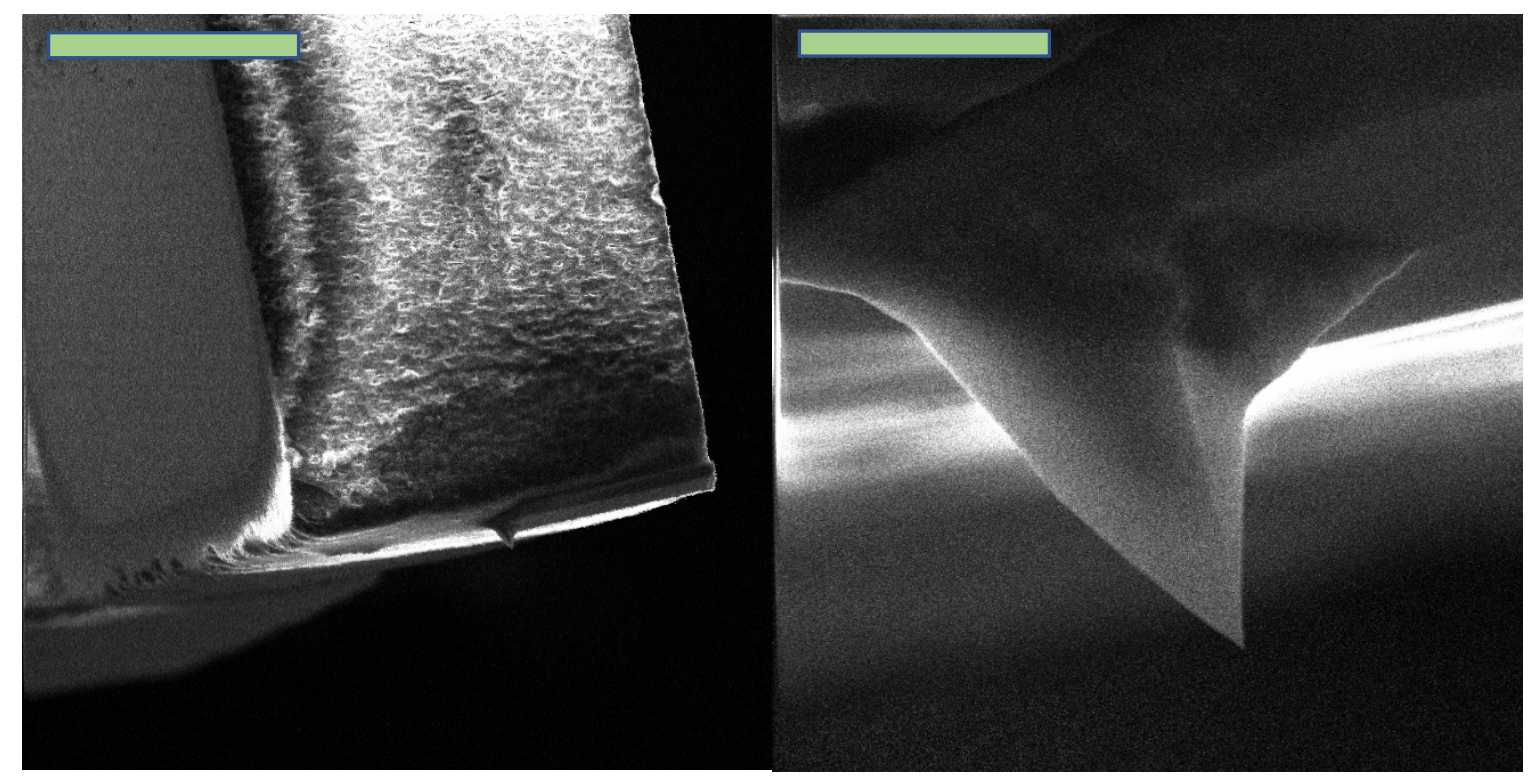

Figure $7 \boldsymbol{L e f t}$, SEM image of AFM cantilever after being epoxied to the bottom of a tuning fork, (green scale bar $\sim 70$ um). Right. Close image of the AFM probe illustrating the geometry (green scale bar $\sim 10 \mathrm{um}$ ).

\section{(2.4) The Tuning Fork Sensor: Theoretical Basis and Calibration}
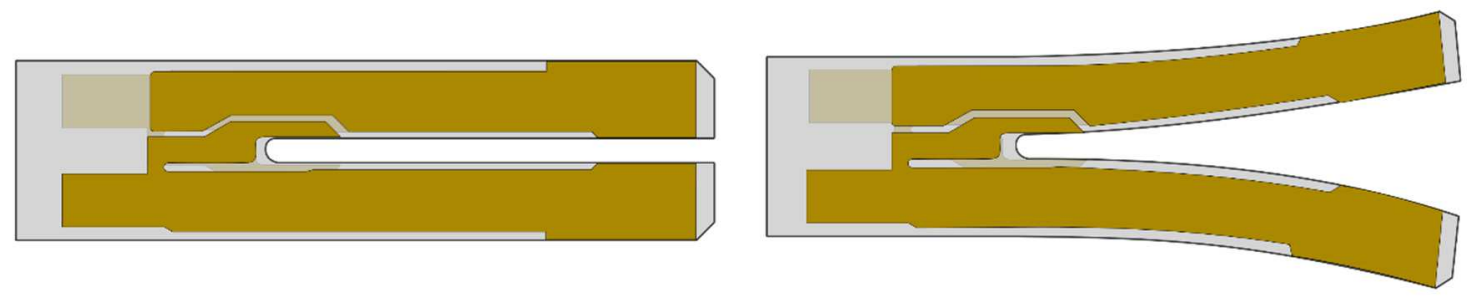

Figure 8 Left) Schematic of quartz tuning fork with zero applied driving voltage Right) Schematic of quartz tuning fork at time $t$, with tines exibiting deformation caused by applied driving voltage $\mathrm{V}$. 
QTF's work especially well as sensors, because they utilize the piezoelectric effect of Quartz to both drive mechanical oscillations and detect tine displacement, purely eclectically. To better appreciate this operate, we can refer to the schematic diagram of the QTF's electrical equivalence circuit shown in figure 9 below. The tuning fork is modeled by the "Butterworth-Van Dyke" or BVD circuit consisting of a series Resistor, Inductor, and Capacitor (RLC) which simulate the oscillator. This oscillator is then placed in parallel with a capacitor $C_{0}$ to account for the inherent capacitance of the physical device [23]. Electrical RLC resonator circuits are often compared with physical oscillators such as the block and spring system because they share the same form of differential equation. In this fashion, the electrical oscillator mode can be related to a mechanical oscillator. It will be shown below that the vibrations of each cantilevered tuning fork tine can be expressed equivalently as a Simple Harmonic Oscillator (SHO), with effective mas and spring constant $\mathrm{m}_{\mathrm{eff}}$ and $\mathrm{K}_{\mathrm{eff}}$, determined by the physical properties of the tuning fork.
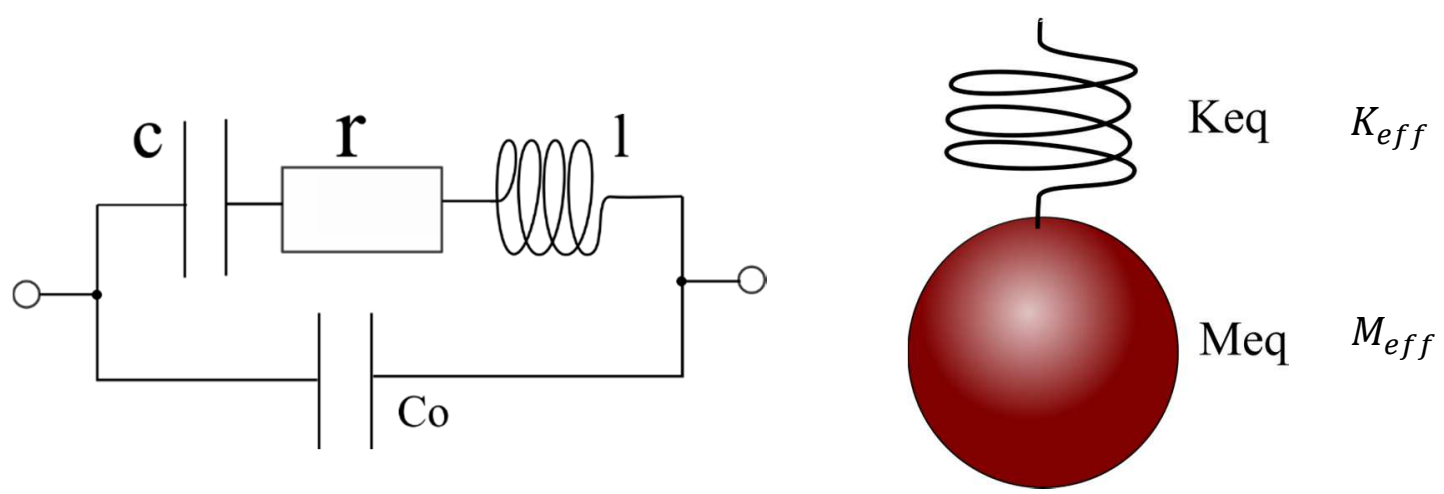

Figure $9 \boldsymbol{L} \boldsymbol{e f t}$ )Schematic representation of quarts tuning fork Butterworth-Van Dyke electrical equivelence citcuit Right) Schematic representation of quartz tuning fork as simple harmonic oscilator, with equivalent mass $M_{e f f}$ and spring constant $k_{e f f}$. 
As expected, the equations of motion (EOM) governing this 'equivalent' SHO is simply

$$
\mathrm{m}_{\mathrm{eff}} \frac{d^{2} u(t)}{d t^{2}}+F_{d}+\mathrm{K}_{\mathrm{eff}} u(t)=F_{\text {ext }}
$$

where $F_{\text {ext }}$ is the external driving force of the form $F_{\text {ext }}=F_{0} e^{(i \omega t)}$, and $F_{d}$ the total damping forces consisting of internal parasitic losses, and tip-sample interactions, expressed as $F_{d}=m_{e q} \gamma \frac{d u}{d t}[17]$. The governing equation for an equivalent electrical model is generated by applying Kirchhoff's laws to the RLC resonator circuit.

$$
L \frac{d I}{d t}+R I+\frac{Q}{c}=V_{\text {ext }}
$$

again $V_{\text {ext }}$ is an external driving voltage of the form $V_{\text {ext }}=V_{0} e^{(i \omega t)}$. we can relate the mechanical and electrical models using their expressions of power. In this way, the mechanical expression is multiplied by velocity such that $P_{\text {ext }}=F_{\text {ext }} \frac{d u}{d t}$, and the electrical equation is multiplied by current to yield $P_{\text {ext }}=V_{\text {ext }} \frac{d Q}{d t}$.

$$
\begin{gathered}
\mathrm{m}_{\mathrm{eff}} \frac{d^{2} u(t)}{d t^{2}} \frac{d u}{d t}+m_{e q} \gamma \frac{d u}{d t} \frac{d u}{d t}+\mathrm{K}_{\mathrm{eff}} u(t) \frac{d u}{d t}=F_{0} e^{(i \omega t)} \frac{d u}{d t} \\
L \frac{d^{2} Q(t)}{d t^{2}} \frac{d Q}{d t}+R \frac{d Q}{d t} \frac{d Q}{d t}+\frac{Q}{c} \frac{d Q}{d t}=V_{0} e^{(i \omega t)} \frac{d Q}{d t}
\end{gathered}
$$

or rearranging both equations to be expressed in terms of kinetic and potential energy leads to

$$
\begin{aligned}
\frac{d}{d t}\left[\frac{\mathrm{m}_{\mathrm{eff}}}{2} \frac{d u^{2}}{d t}\right]+m_{e q} \gamma \frac{d u^{2}}{d t}+\frac{d}{d t}\left\lfloor\frac{\mathrm{K}_{\mathrm{eff}}}{2} u(t)^{2}\right\rfloor & =F_{0} e^{(i \omega t)} \frac{d u}{d t} \\
\frac{d}{d t}\left[\frac{\mathrm{L}}{2} \frac{d Q^{2}}{d t}\right]+R \frac{d Q^{2}}{d t}+\frac{d}{d t}\left\lfloor\frac{1}{2 C} Q(t)^{2}\right\rfloor & =V_{0} e^{(i \omega t)} \frac{d Q}{d t}
\end{aligned}
$$

clearly

$$
\frac{\mathrm{L}}{2} I^{2}=\frac{\mathrm{m}_{\text {eff }}}{2} \dot{u}^{2}, m_{e q} \gamma \dot{u}^{2}=\mathrm{R} I^{2}, \frac{1}{2 C} Q(t)^{2}=\frac{\mathrm{K}_{\text {eff }}}{2} u(t)^{2} \text {, and } F_{\text {ext }} \dot{u}=V_{\text {ext }} I .
$$


Because of the piezoelectric nature of quartz, a deflection in the tines generates a charge separation Q. In the SHO model this Charge corresponds to a linear relationship between Q and $u$, where $\alpha$ is the piezo-electric mechanical coupling constant [23]. Mathematically,

$$
\begin{aligned}
& Q=2 \alpha u, \\
& I=2 \alpha \dot{u}
\end{aligned}
$$

where the factor of 2 accounts for the tuning fork containing 2 tines. Plugging this into the kinetic and potential equalities above yields the desired expressions.

$$
\begin{aligned}
& L=\frac{\mathrm{m}_{\text {eff }}}{2 \alpha^{2}} \\
& c=\frac{2 \alpha^{2}}{\mathrm{~K}_{\mathrm{eff}}} \\
& R=\frac{\mathrm{m}_{\mathrm{eff}} \gamma}{2 \alpha^{2}} \\
& V_{\text {ext }}=\frac{F_{\text {ext }}}{\alpha}
\end{aligned}
$$

Because the general solution to the differential equation governing the SHO yields standard solutions $u(t)=u_{0}(t) e^{i(\omega t+\theta)}, 2.4-5 \mathrm{~b}$ implies

$$
I_{0}=i 2 \alpha \omega u_{0}
$$

meaning that if $\alpha$ can be determined, the amplitude of oscillation can be found from a measurement of the current generated between the tuning fork leads. Evidently, $\alpha$ is related to capacitance and effective spring constant through the expression 2.4-6b. However, the capacitance $\mathrm{C}$, (as well as $\mathrm{R}, \mathrm{L}$, and $C_{0}$ ) can be determined by fitting experimental current spectra measurements to a model derived from the transfer function $Y_{\text {total }}(\omega)$ of the BVD circuit (outlined above in figure 9) and is plotted bellow in figures 10 and 11. This transfer 
function is related to the complex systems impedance $Z_{\text {total }}$, where $\frac{1}{Z_{\text {total }}(\omega)}=Y_{\text {total }}(\omega)=$ $\frac{I(\omega)}{V}$.

$$
\begin{aligned}
& \frac{1}{Z_{\text {total }}}=\frac{1}{Z_{R L C}}+\frac{1}{Z_{C_{0}}} \\
& \frac{1}{Z_{\text {total }}}=\frac{1}{R+i \omega L-\frac{i}{\omega c}}+\frac{1}{\frac{-i}{\omega C_{0}}} \\
& \frac{1}{Z_{\text {total }}}=\frac{\frac{-i}{\omega C_{0}}+R+i\left(\omega L-\frac{1}{\omega c}\right)}{\frac{-i}{\omega C_{0}}\left(R+i\left(\omega L-\frac{1}{\omega c}\right)\right)} \\
& \frac{1}{Z_{\text {total }}}=\frac{\omega C_{0}\left(\frac{R}{i}+\omega L-\frac{1}{\omega}\left(\frac{1}{C_{0}}+\frac{1}{c}\right)\right)}{R+i\left(\omega L-\frac{1}{\omega c}\right)}
\end{aligned}
$$

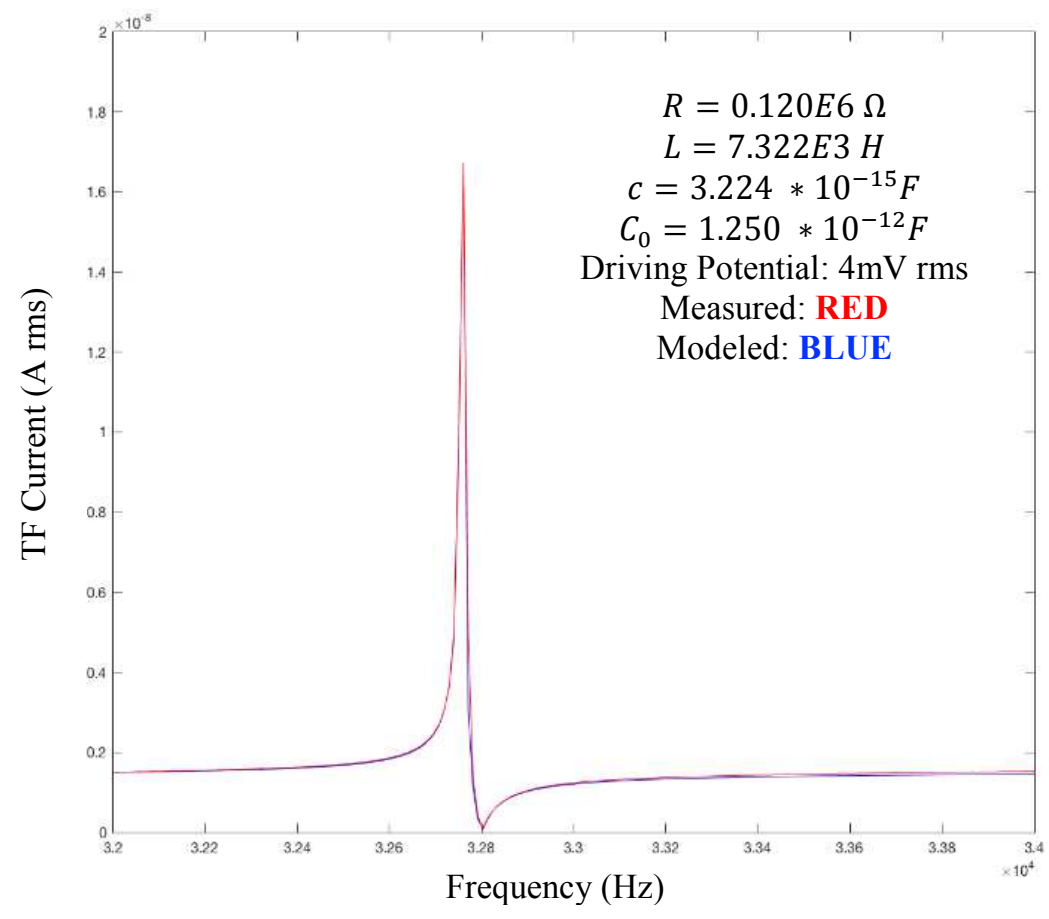

Figure 10 A Plot of the modeled tuning fork current $Y_{\text {total }}(\omega) / \mathrm{V}$ (blue) along with measured current (using lock in amplifier) to determine fit parameters. 


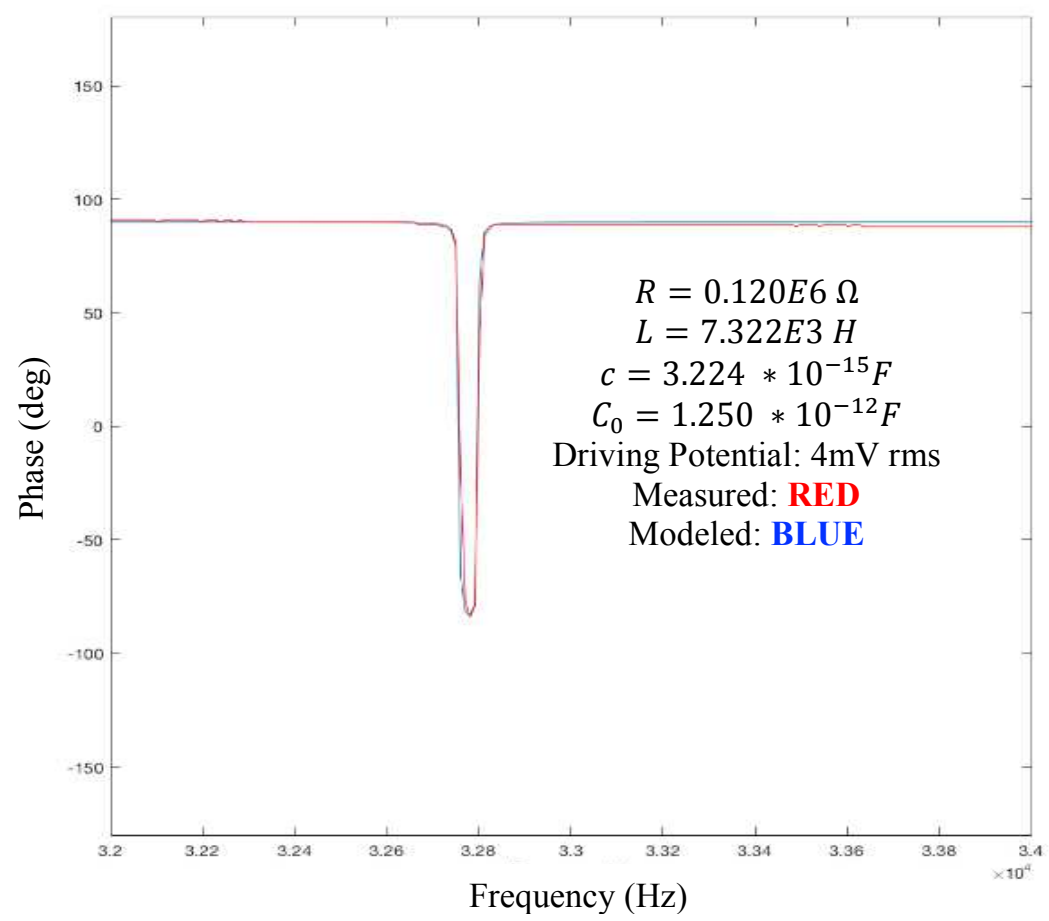

Figure 11 A plot of the modeled tuning fork phase $\tan ^{-1}\left(\frac{\text { real }\left(Y_{\text {total }}\right)}{\operatorname{img}\left(Y_{\text {total }}\right)}\right)$ (blue) along with measured phase (using lock in amplifier) (red) to determine fir parameters.

The plots in figure 10 and 11 show that the modeled current spectrum (blue) is in excellent agreement with the measured current (red) (for this particular trial) when appropriate fit parameters are selected as follows,

$$
\begin{gathered}
R=0.120 E 6 \Omega \\
L=7.322 E 3 H \\
c=3.224 * 10^{-15} \mathrm{~F} \\
C_{0}=1.250 * 10^{-12} \mathrm{~F}
\end{gathered}
$$

Having found that the oscillator capacitance is equal to $3.224 * 10^{-15}$ Farads in this case, the piezo-electric mechanical coupling constant $\alpha^{2}$ is constrained as $\alpha^{2}=$ 
$\sqrt{\frac{\left(3.224 * 10^{-15}\right) \mathrm{K}_{\mathrm{eff}}}{2}}$ so long as an effective mass $\mathrm{m}_{\mathrm{eff}}$ and spring constant $\mathrm{K}_{\mathrm{eff}}$ are found that relate cantilevered beam vibrations to a SHO model. As it happens, this relationship is well-known, and has general solutions $\mathrm{m}_{\mathrm{eff}}=0.2427 \rho L W T$, and $\mathrm{K}_{\mathrm{eff}}=\frac{E W}{4}\left(\frac{T}{L}\right)^{2}$, where E is the Young's modulus of quartz; these expressions are derived in appendix A and B below.

\section{(2.5) Decoupling Forces: The Need for Frequency Modulation}

The SANM focuses on metrology pertinent to the characterization of elastic and dissipative interaction forces between the probe and sample (among other properties). It is advantageous then to decouple elastic from dissipative interactions in the tuning fork detection scheme. As discussed in appendix D, the dissipative interactions between the probe and sample can be thought of as resulting from an additional 'damping coefficient' $\left(\gamma_{\text {int }}\right)$. Likewise, the elastic components may be interpreted as an additional interaction spring constant' $\left(\mathrm{K}_{\mathrm{int}}\right)$. Therefore, when the tuning fork is interacting with the sample, its resonant frequency $\omega_{0}{ }^{\prime}$ can be calculated as follows

$$
\omega_{0}^{\prime}=\sqrt{\frac{\left(\mathrm{K}_{\mathrm{eff}}+\mathrm{K}_{\mathrm{int}}\right)}{\mathrm{m}_{\mathrm{eff}}}}
$$

where $\mathrm{K}_{\mathrm{eff}}$ is the effective spring constant of the bare tuning fork tine, $\mathrm{m}_{\mathrm{eff}}$ is the effective mass, and again, $\mathrm{K}_{\mathrm{int}}$ is the interaction spring constant. When the tuning fork probe is away from the sample $\mathrm{K}_{\mathrm{int}}=0$, and a spectrum of the tuning forks mechanical amplitude near resonance is similar to that which is displayed in Figure 12a). However, as the probe begins interacting, $\mathrm{K}_{\mathrm{int}}$ will (by definition) be non-zero for samples exhibiting elastic or 
viscoelastic properties. This resonant frequency shift poses a problem using a synchronous detection system at constant frequency such as a lock in amplifier (the latter is generally required as the tuning fork current signal response is typically on the order of nA). To outline the problem, let's begin by analyzing the simpler idealized case where the probe interacts purely elastically (not viscously) with the sample. In the constant frequency case, the tuning fork is electrically driven and detected at a single frequency $\omega_{0}$ (typically at or near the resonance frequency when positioned away from the sample). As the probe begins interacting, $\mathrm{K}_{\text {int }}$ may increase and drag the new resonance frequency $\omega_{0}{ }^{\prime}$ away from the excitation/detection frequency $\omega_{0}$ which is displayed in figure 12b). Graphically, this means the amplitude is measured as the point where the black spectrum coincides with the blue vertical bar (clearly much lower than in case 1A). This shift drives the system out of resonance, and the elastic interaction has manifested as a perceived decrease tuning fork amplitude at $\omega_{0}$. Conversely, we could think about the outcome of the probe interacting purely viscously. Clearly the amplitude would decrease while resonant frequency remains the same as seen in figure 12c) which (from the lock-in point of view) is indistinguishable from the previous elastic case. In this way, monitoring the tuning fork amplitude alone is not sufficient to determine if a decrease in the detected tuning fork amplitude is a result of a dissipative (viscus) interaction with the sample, or an elastic interaction that has simply shifted the resonance frequency. 
A)

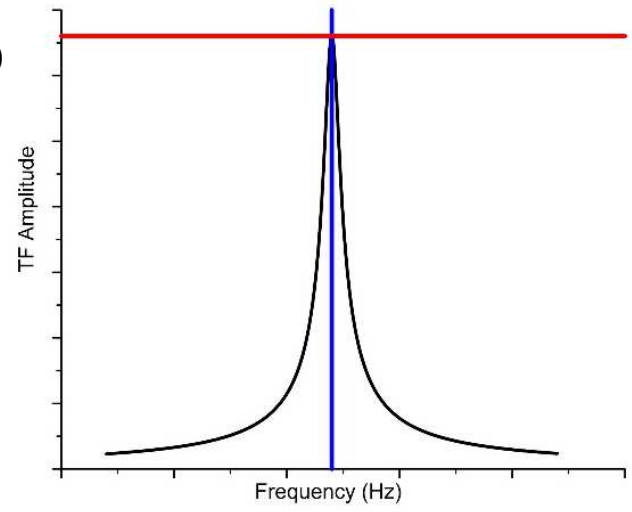

C)

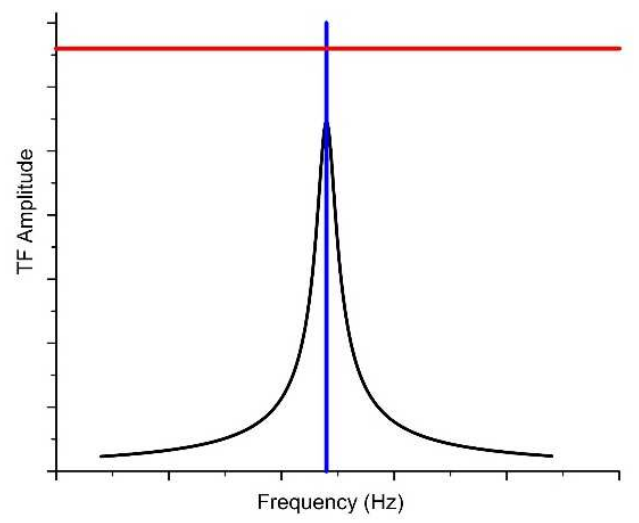

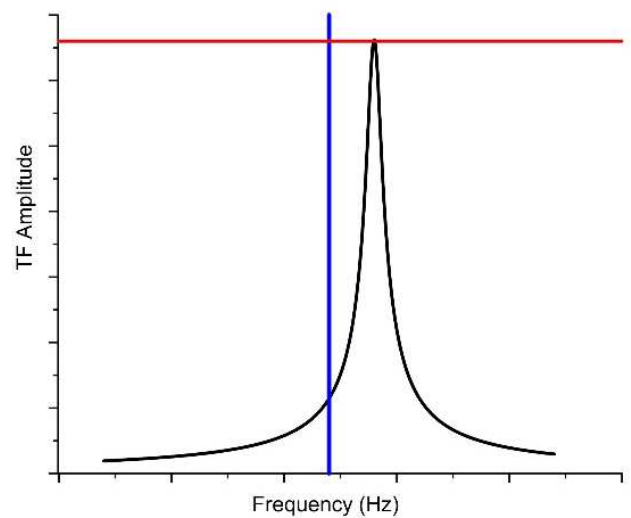

B)

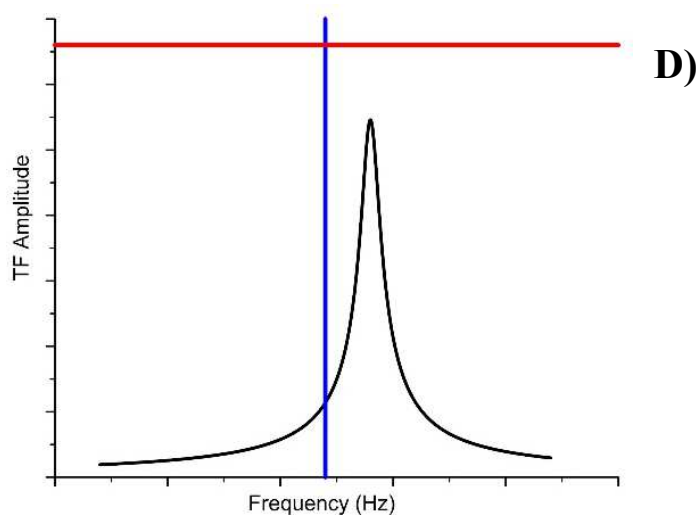

Figure 12 Top Left A) spectrum showing the mechanical resonance of the tuning fork. The blue line denotes the frequency at resonance, (when measured with the tip away from the surface) while the red bar signifies the peak amplitude level. Top Right B) In an Idealess case where only elastic forces are present, the resonance frequency shift as illustrated. Bottom Left C) In an idealized case, there only viscus (damping) interactions are present, the amplitude at resonance decreases from its previous maximum. Bottom Right D) A typical viscoelastic interaction where the tuning fork experiences both a shift in resonance frequency, and a decrease in driving amplitude.

\section{(2.6) Retrieving Resonance Frequency Shift and Resonance Frequency Amplitude}

\section{from Constant Frequency Measurements}

because both elastic and inelastic interactions are coupled to the probes amplitude (in the constant frequency regime), additional information is required to investigate each parameter independently. This is accomplished by supplementing the oscillators amplitude information with mechanical phase measurements. These mechanical measurements are 
derived from the tuning forks electrical phase and amplitude response (the details of this process are presented later in equation 2.8-1 of "Acoustic Sensors" and derived in Appendix C). Knowing both amplitude and phase, the EOMs can be manipulated (see appendix E), to yield the elastic component resulting from the interaction spring constant $K_{\text {int }}$, where

$$
K_{\text {int }}=\frac{F_{\text {ext }} \cos \varphi}{U}-K_{e f f}\left(1-\frac{\omega^{2}}{\omega_{0}^{2}}\right) .
$$

Similarly, the dissipative interaction stemming from the damping coefficient $\gamma_{\text {int }}$ can be calculated using

$$
\gamma_{i n t}=\left(\frac{F_{e x t} \sin (2 \varphi)}{2 M_{e f f} U \omega}\right)-\gamma_{e f f},
$$

(again derived in appendix E). Here $\varphi$ is the mechanical phase with respect to the driving force $F_{\text {ext }}$ (equation 2.4-6d), $\omega_{0}$ is the tuning forks resonance frequency, and $\omega$ is the chosen driving/detection frequency.

\section{(2.7) Frequency Modulation Method}

Frequency Modulation is a second method implemented in this microscope to decouple elastic and dissipative interactions by continually updating the driving/detection frequency to maintain resonance regardless of real time changes in interaction spring constant $K_{\text {int }}$. Therefore, because the system is always at resonance, a decrease in the tuning forks amplitude can be largely attributed to dissipative viscus interactions. Conversely, changes in resonance frequency (with respect to $f_{0}$ taken far from the surface) are attributed to elastic interactions. Hence, for the case of frequency modulation, the 
interaction spring constant $K_{\text {int }}$, and damping coefficient $\gamma_{\text {int }}$ are calculated from the EOMs as

$$
\mathrm{K}_{\mathrm{int}}=\mathrm{K}_{\mathrm{eff}}\left(\left(\frac{f_{0}{ }^{\prime}}{f_{0}}\right)^{2}-1\right)
$$

and

$$
\gamma_{\text {int }}=\gamma\left(\frac{f_{0} u_{0}}{f_{0}^{\prime} u^{\prime}}-1\right)
$$

respectively (see appendix D), where $u_{0}$ is the magnitude of the tuning forks amplitude away from the sample, and $u^{\prime}$ is the new amplitude due to dissipative interactions. Figure 13 'left' shows schematically how this frequency modulation scheme is implemented within our microscope.
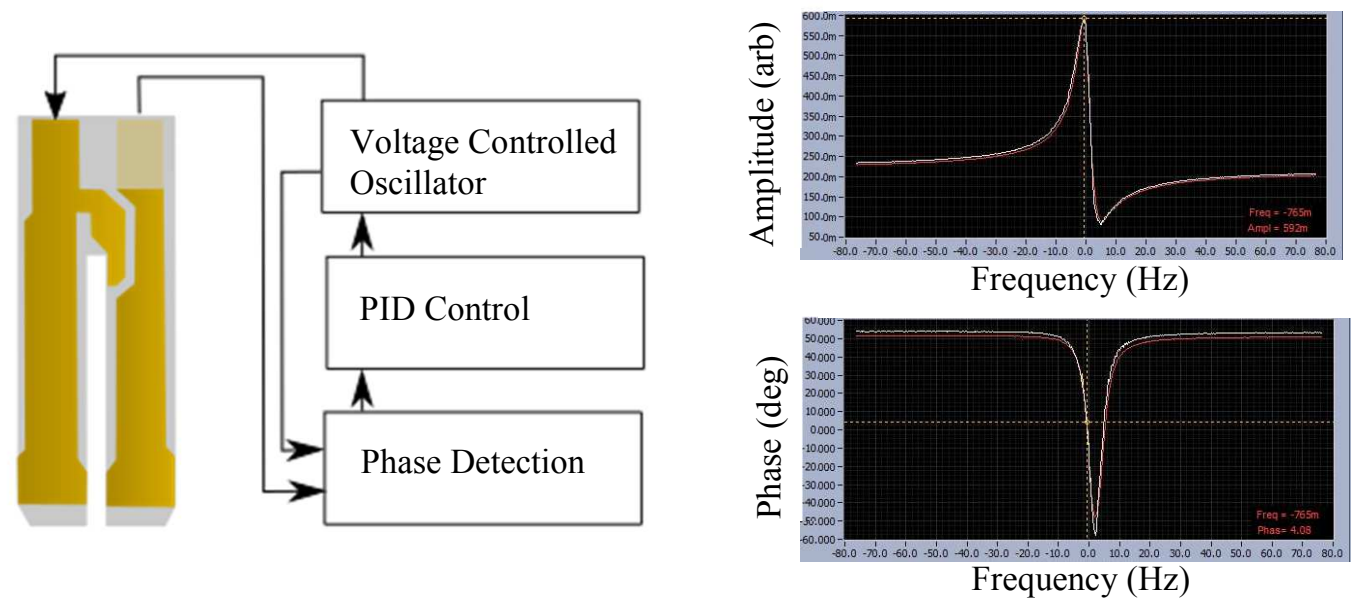

Figure 13 Left) schematic displaying how the frequency modulation scheme is implemented within our microscope. Right Above) Screen capture from the Nononis tuning fork driving software, showing a spectrum of tuning fork amplitude as a function of frequency. Right Below) Screen capture from the Nanonis tuning fork driving software showing the phase of the tuning forks current response (measured with respect to the driving potential) as a function of frequency. 
First, a variable oscillator is implemented to excite the tuning fork through resonance, while a lock in amplifier measures the amplitude and phase (with respect to the driving signal); typical results are displayed in figure 13 'right'). If there were no internal capacitance, electrical resonance would take place when the current from the quartz tuning fork is exactly in phase with the driving signal. However, due to the device's intrinsic capacitance, the phase may not be exactly zero. Instead, the frequency corresponding to the maximum amplitude is used to determine resonance, the phase associated with this frequency is herein referred to as the 'reference phase' (perhaps 20 degrease or so). With the reference phase ascertained, the system may now be switched into Frequency modulation. In this regime, the system may interact with viscoelastic forces just as before, however now as $K_{\text {int }}$ becomes non-zero and drags $\omega_{0}{ }^{\prime}$ away from $\omega_{0}$, the phase will also begin to shift away from the reference value as the system will no longer be in resonance. Therefore, the non-zero difference between the instantaneous phase and the reference phase is used as an input for the Proportional-Integral-Derivative (PID) control. This PID control is then used to modulate the QTF's driving frequency, effectively doing whatever is necessary to minimize the difference between the phase and its set point, thereby bringing the system back into resonance. This frequency $\omega_{0}{ }^{\prime}$ is tracked and used in equation 2.7-1 and 2.7-2 to find the dissipative and elastic components.

\section{(2.8) Acoustic Sensors}

The piezoelectric properties of QTF's mean an electrical response is generated due to mechanical oscillations in the tuning fork tines. However, from the BVD model, it was 
shown that the real internal capacitance of wires, and connections cause the QTF's electrical response to be indirectly related to the tines mechanical displacement. However, analyzing the electrical model, it was found that this mechanical displacement is directly related to current flowing in the RLC portion of the ideal circuit (via the piezoelectromechanical coupling constant derived in 2.4-5a). In this way (see appendix C), the current proportional to the true mechanical displacement can be decoupled using the electrical amplitude in conjunction with electrical phase to yield

$$
\begin{aligned}
& I_{m}(\omega)=\sqrt{I_{e}{ }^{2}-2 \omega C_{0} V_{0} I_{e} \sin \theta_{e}+\left(\omega C_{0} V_{0}\right)^{2}}, \\
& \theta_{m}(\omega)=\arg \left[I_{e} \cos \theta_{e}+j\left(I_{e} \sin \theta_{e}-\omega C_{0} V_{0}\right)\right] .
\end{aligned}
$$

Again, the real capacitance $C_{0}$ is found by first fitting the experimental current amplitude and phase to the model (see figures 10 and 11), while $\mathrm{I}_{\mathrm{e}}$ and $\theta_{\mathrm{e}}$ the experimental values of the TF current amplitude and phase to be measured by the lock-in amplifiers respectively. The difference between the tuning forks total current response $I_{e}$ (measured directly), and RLC current $I_{m}$ (proportional to mechanical displacement), can be readily seen when plotted on the same axis. Not only is the electrical spectrum asymmetrical and shifted vertically (making accurate absolute displacement measurements difficult in real time), but it also displays a resonance peak that is shifted several $\mathrm{Hz}$ with respect to the true mechanical peak. 

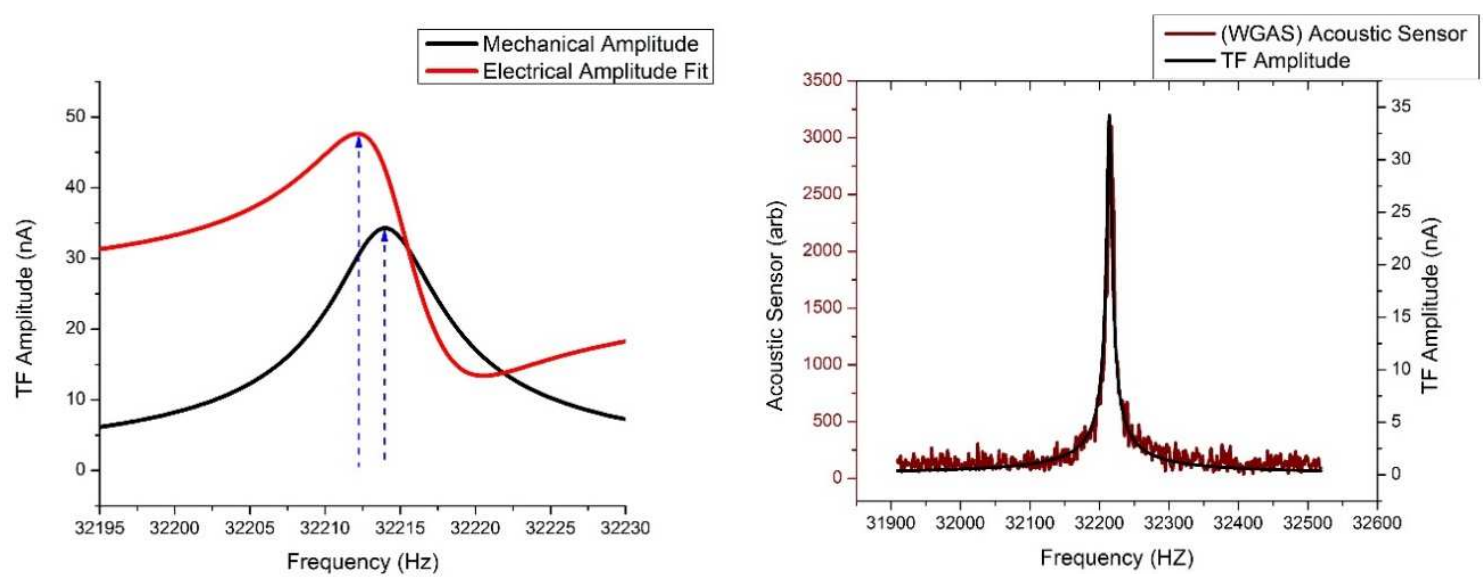

Figure 14 a) Left: Tuning forks total current spectral response (red trace) and its calculated (using expression 2.8-1) spectral response when the parallel capacitance is factored out to obtain the current response from the RLC portion, the latter being proportional to the tuning fork's mechanical displacement. b) Right: comparison of the WGAS acoustic transducers response, versus the calculated tuning forks mechanical displacement.

\section{(2.9) The WGAS Technique}

The above decoupling technique for the probes amplitude cannot be measured directly from the lock-in, rather it requires computational analysis. For many metrology experiments, real time knowledge of the probes mechanical displacement is imperative. One solution consists of implementing an electrical circuit aimed at compensating for the distortion caused by capacitance [24]. However, a more elegant solution is implemented here wherein a piezoelectric acoustic transducer is used to monitor the QTF's mechanical oscillations directly. In this configuration, the tuning fork sensor is secured to the microscope using a collet style clamp which is machined into a 'fixture' (seen in figure 15) that I recently redesigned, (and had fabricated in cast iron by Alex Challey). When the QTF is excited electrically it oscillates, creating small vibrations that are transmitted through the fixture. Placing an acoustic transducer in contact with this fixture generates an electrical 
signal directly proportional the probes mechanical oscillations, therein allowing real-time measurements of the probe's displacement. Figure $14 \mathrm{~b}$ further validates this approach displaying the acoustic sensor's response as well as the probe's mechanical displacement, (calculated using equation 2.8-1) and demonstrates excellent agreement between the signals.

The purpose for redesigning the 'fixture' was to decrease the impedance of acoustic signals which are engendered at the tuning fork, then enter the fixture through the collet clamp, and travel to the opposite end of the part where they are ultimately detected using the WGAS sensor. A previous iteration of the microscope utilized a non-compensated piezoelectric tube for scanning and controlling the relative probe sample spacing. In this previous design, the QTF was attached to a plastic clamp, which was glued to the piezoelectric tube, which was itself glued to a ceramic colder, all before finally being attached to the microscope head using a clamping retainer. Each of these interfaces introduced a change in impedance which could cause acoustic reflections and degrade the final signal. The new one-piece design eliminates these interfaces, and may even provide compensation from thermal drift due to its nested cylinder design inside of the "whispering gallery' (a component in the SPM head supporting the 'fixture', see figure 1f) composed of the same material.

Even with these improvements, the sensitivity requires further refinement. The optimal signal to noise ratio (SNR) was documented at 2,500 (noise signal equal to $2 \%$ of the voltage signal), with typical SNR readings near 225 (6\%). Many parameters affect this 
peak sensitivity and noise level, including transducer placement, and the collets applied clamping force to affix the tuning fork to the fixture; both are discussed below.
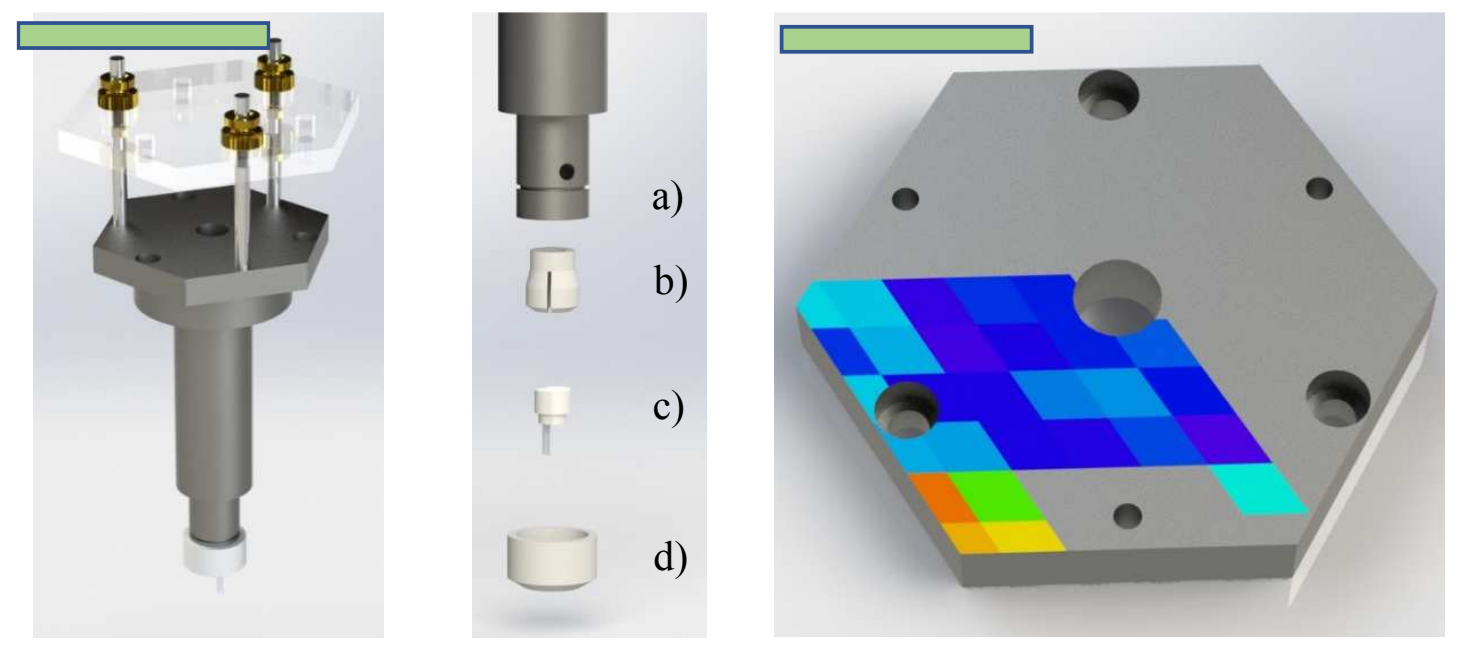

Figure 15 Left) Diagram showing the fixture and components which holds the tuning fork. The top surface of the fixture is where the acoustic sensor is placed. Also pictured are the 3 threaded rods and brass thumb screws which tighten against the acrylic plate that are used clamp the WGAS sensor to the fixture (green scale bar $\sim 6.5 \mathrm{~cm}$ ). Middle) Exploded diagram specifically showing a) fixture, b) collet, c) Macor and tuning fork, d) collet clamp. Right) A false color image is overlaid on top of this surface indicating the location of largest sensitivity in orange and yellow, whereas the location of least sensitivity appears in dark blue (green scale bar $\sim 3.7 \mathrm{~cm})$.

Best sensitivity is achieved by placing the transducer at a location coinciding with a mechanical resonant antinode on the microscope head. This head is composed of multiple components including the base 'plate', 'Whispering Gallery', and cast iron 'fixture' (see exploded diagram, figure 15). The net resonant characteristic for this interacting system is complexly related to the individual components attributes, and the applied forces connecting them. This research group has already explored optimizing the microscope's resonance response by modifying the 'whispering gallery's' geometry, to match the tuning forks mechanical resonance, and increase the WGAS signal to noise ratio [25]. However, 
the sensitivity can also be increased by determining the optimal placement of the WGAS acoustic sensor on the fixture (atop the assembled microscope head). This was accomplished experimentally. A series of frequency sweeps were acquired with the sensor placed at various locations on the fixture. A grid was etched onto the top surface of the fixture (where the acoustic transducer is placed), and the acoustic response was measured at each element for a given constant driving amplitude throughout. Figure 15 right displays a false color image indicating sensitivity at various locations (with red indicating highest intensity and violet the smallest). Clearly the greatest sensitivity is achieved near the corners of the hexagonal fixture. Another parameter which seems to have a large impact on the sensitivity of the WGAS acoustic sensor is the tuning forks clamping force. As the collet clamp is screwed down (figure 15, middle, $d$ )), it is compressed against an internal taper within that cast iron fixture, applying an adjustable clamping force against tuning fork. It was noticed that for a given tuning fork and drive voltage, the quality factors of both the electrical QTF, and WGAS acoustic signals, could vary widely depending on the torque applied to the component ' $\mathrm{d}$ '. To determine an optimal torque value, a QTF was excited with constant driving potential, and spectra were acquired at a variety of clamping forces. Unfortunately, the torque values used were well below the measurable range on commonly available torque wrenches, however a scheme was devised to determine the optimal clamping force by measuring the angular displacement of the clamping nut (which is related in some unknown way to the clamping force). In this case, the position wherein the nut first contacts the uncompressed collet is arbitrarily defined as the angular zero. 

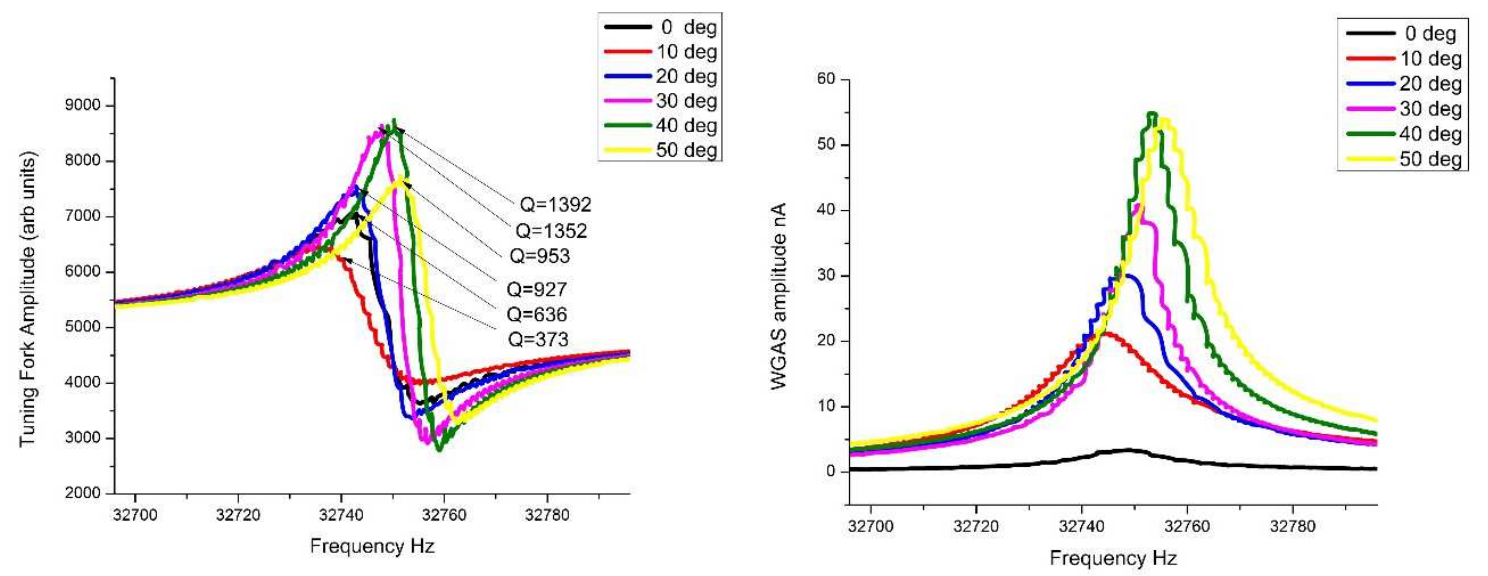

Figure 16 Sensitivity to different ways of mounting the QTF. Left: Tuning fork electrical response at a variety of collet nut (piece 'd' in Fig. 14)angular displacements including associated quality factors. Right: WGAS spectral response at a variety of collet nut angular displacements.

It was found that a peak signal on both the tuning fork and acoustic signal could be attained at a torque value corresponding to 40 degrees of rotation on the collet nut. It is somewhat surprising to see the quality factors change by more than $350 \%$ with slight variations in clamping force. This shows that the connection between the tuning fork and microscope is one channel of energy dissipation. Hence, the way the QTF is clamped affects not only the capacitance of the QTF, but also, and principally, its mechanical response. That is, the clamp holder becomes part of the QTF, and thus affects the way the QTF responds. The WGA signal confirms that this clamp-QTF interaction is dissipative (the frequency shift is negative and the peak amplitude decreases, shown in Figure. 16. Without the WGAS signal one could have blamed capacitive effects to the changes in the QTF resonance frequency response. In passing, this demonstrates the additional metrology capabilities offered by The WGAS technique. 


\section{(2.10) The SANM Technique}

This microscope employs a second acoustic sensor placed directly below the sample to monitor acoustic emissions generated via interactions between the laterally oscillating probe and sample during an 'approach retraction' sequence (discussed at length below). This sensor is referred to as the Shear-Force Acoustic Near Field Microscopy sensor, or SANM sensor for short. The idea was introduced by our research group in a 2005 paper published in Review of Scientific Instrument [26]. Wherein a correlation was found between viscoelastic probe sample interaction forces, and acoustic emissions (measured by the piezoelectric SANM transducer). While the sensor is designed to detect probe sample interactions, it is also sensitive enough detect vibrations of the tuning fork probe in air, when aggressively driven a few microns above the substrate.

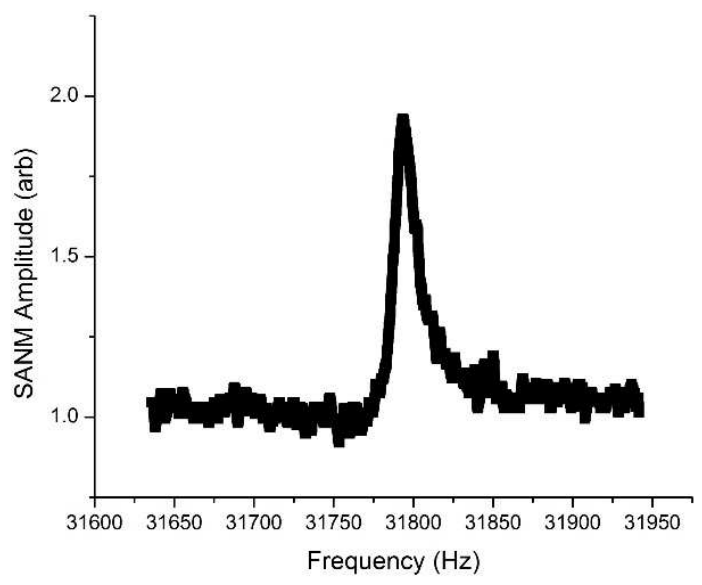

Figure 17 Above: A spectrum showing the SANM response when a driven tuning fork is swept through resonance with the tip placed a few microns above the sample (which is placed in intimate mechanical contact with the sensor) 
This ability is often utilized before beginning approach-retraction experiments to ensure sensor reliability, by sweeping the tuning fork through resonance and evaluating the SANM's response. An example of this acoustic response can be seen in figure 17 above.

\section{(2.11) Acoustic Sensor Characterization}

One desirable property of an acoustic sensor is a flat response in the frequency band of interest. This ensures a change in recorded amplitude is the result of a physical change in the system rather than gain fluctuations manifesting from a shift to a different part of the frequency band. To characterize this, a piezo stack (EBL\# 2 Shear Mode Plates) with a known flat response in the desired range, was used to excited the SANM and WGAS sensors directly, and measure the response power spectrum which is displayed in figure $18 \mathrm{~b}$ and $19 \mathrm{c}$. Because the WGAS sensor is used to measure the tuning fork displacement in-situ, the spectral response was also measured using the piezo stack to excite the fixture shown in figure $18 \mathrm{a}$. In each case the sensors exhibited a response within $+15 \mathrm{db}$, and $20 \mathrm{db}$ when measured over a frequency band of $20 \mathrm{KHz}$ centered around $32.7 \mathrm{KHz}$, and under $\mp 5 \mathrm{~dB}$ in the typically used band between $31 \cdot 0-32.7(\mathrm{KHz})$.
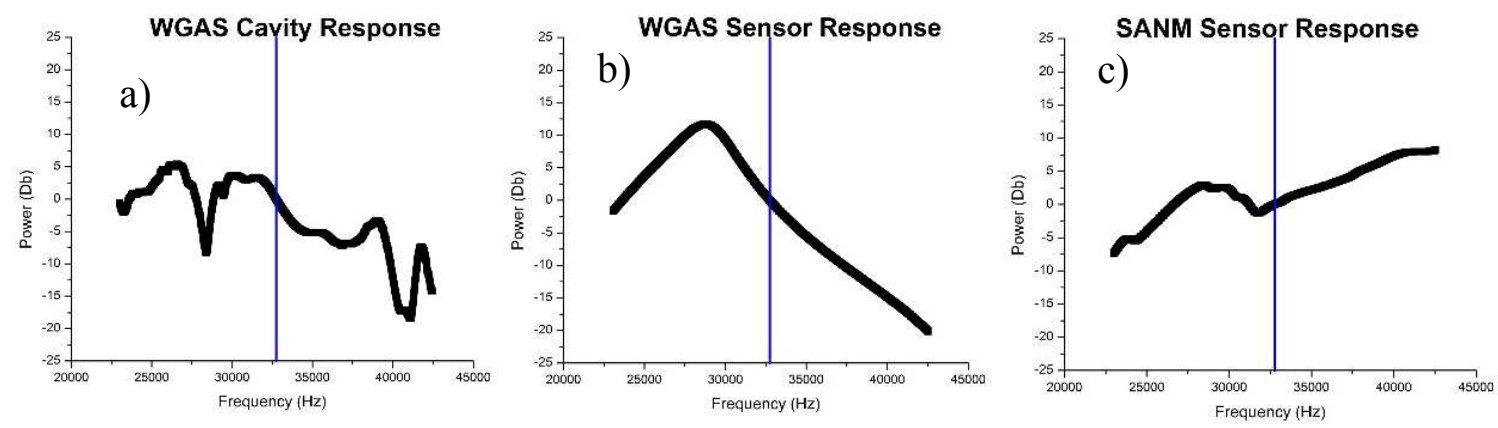

Figure 18: Characterizing the acoustic sensors directly. Note graph displays power relative to the resonance frequency, with domain extending between $22.25 \mathrm{k} \mathrm{Hz}$ and $42.55 \mathrm{k} \mathrm{Hz}$. 


\section{(2.12) Control of the Probe's Vertical Position}

Having discussed the suit of sensors implemented in this Shear-Force Acoustic Near-Field Microscope, Special attention is now paid to its positioning and control mechanisms. Piezo tubes (with a stylus probe attached) are commonly used in open loop configurations for scanning applications where a fast frequency response is critical for driving feedback controls. However, these tubes do suffer from hysteresis and creep, meaning that it is difficult to know precisely the probe's location based on the driving signal. Hysteresis can be reduced, through characterization and compensation of the driving signal. However, this approach requires a calibration method that precisely measures the true $\mathrm{Z}$ height, such as optical interference for example. Still, this correction method constitutes an approximation. Because the hysteresis varies with the distance traveled, and the starting location, an infinite number of characterized hysteresis curves would theoretically be required to fully compensate the system.

For surface metrology applications where an accurate account of the probe displacement is imperative, a closed loop piezo drive system is preferred. For this purpose, the Mad City Labs (MCL) Nano-Z50HS was used. This piezo nanopositioner has $50 \mu \mathrm{m}$ of travel, and its internal closed loop control allows compensation for hysteresis and creep with an expected resolution of $0.1 \mathrm{~nm}$ (if used with a perfectly low noise driving signal). In this case, the closed loop occurs locally at the MCL Nano drive controller. This method is simple, and requires less resources of the Field Programmable Gate Array (FPGA) (rather than using the FPGA to preform closed loop control); in this approach the FPGA is required to only supply a reference signal to the controller. However, this simplicity does come at 
some expense. The true system status is determined by the controller which minimizes error between the provided reference (drive signal) and a measured sensor value (strain gauge in this case), meaning they may not be exactly equal. Additionally, to completely characterize this complex system, there would ideally be a 'perfect' way to measure the stages displacement, and correlate this to the driving signal. However, no 'perfect' method exists. While it is possible to infer this displacement by measuring the response of another sensor (for example a strain gauge in this case), we do not know the sensors exact response or characterization parameters. In this way, the task of complete characterization quickly becomes a control theory problem beyond the scope of this physics thesis. Therefore, the objective here is to minimize the driving signal noise as much as possible, and draw an upper limit on the variance based on theoretically converting the drive signal to a displacement value (assuming an infinite bandwidth frequency response). These noise measurements will first be presented informally in the time domain, looking at maximum pk-pk noise. A more thorough analysis will follow considering improvements in the noise power spectral density.

The MCL nano positioner's $50 \mu \mathrm{m}$ travel is controlled by a $\mp 10 \mathrm{~V}$ analog drive signal, equivalent to $400 \mu \mathrm{V} / \mathrm{nm}$ displacement, which can be a problem with a noisy analog drive signal. The 16-bit digital to analog converter on the PCIE-7852R FPGA card claims $305 \mu \mathrm{V}$ resolution with $150 \mu \mathrm{V}$ noise (DC to $1 \mathrm{MHz}$ ), this signal is then passed down a $5 \mathrm{~m}$ length of cable before reaching the breakout box. After the $5 \mathrm{~m}$ cable, the maximum noise level measured $90 \mathrm{mV}$ pk-pk (see figure 19). If this were converted into mechanical displacement, it theoretically could correspond to roughly $225 \mathrm{~nm}$ in variation, 
significantly above a tolerable maximum for a probing microscope. To minimize noise, the $5 \mathrm{~m}$ cable was replaced with a $1 \mathrm{~m}$ cable yielding a significant improvement at $24 \mathrm{mv}$ pk-pk.

These direct time domain measurements can be seen below in figure 20 .

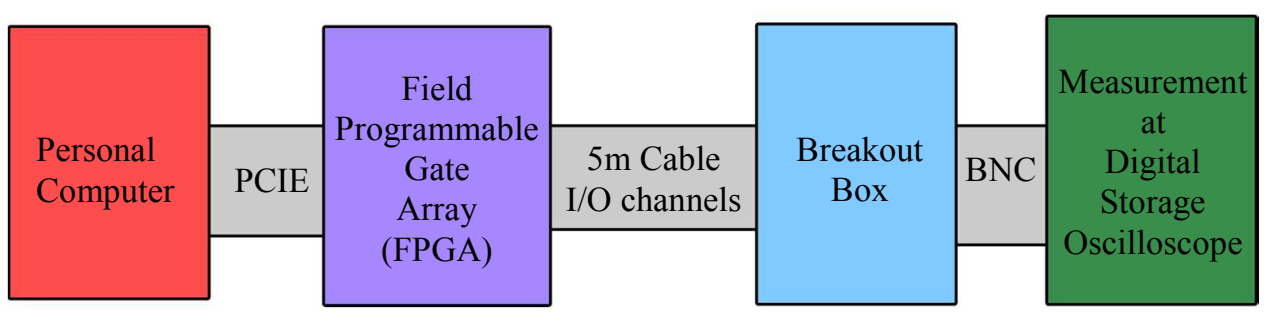

Figure 19 Block diagram showing the elements involved in measuring FPGA noise. The signal emanates with the user at the personal computer, which is connected to the FPGA card via the PCIE interface. The analog signal is then sent from the FPGA board, down the length of cable ( $5 \mathrm{~m}$ here), before reaching the breakout box. The final signal is measured at the Breakout boards BNC output terminals using a digital storage oscilloscope.
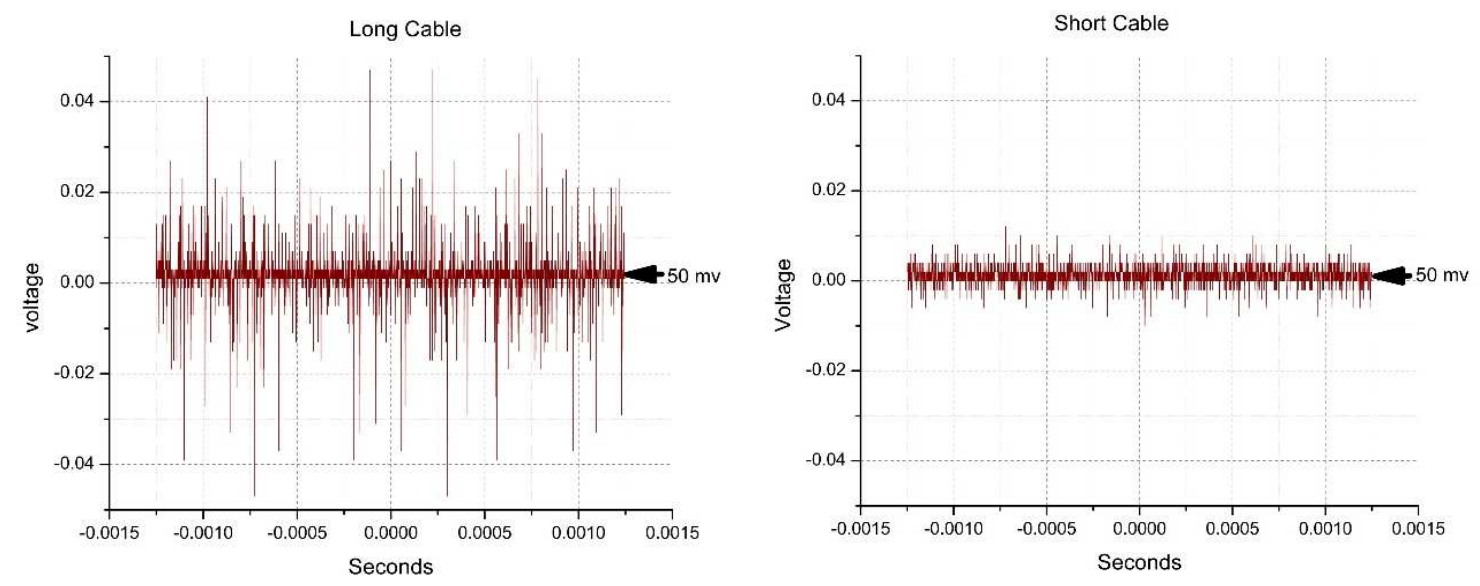

Figure 20 Left) Osciloscope measurment of the FPGA ADC noise level at 50mV offset and $5 \mathrm{~m}$ long cable. Right) Osciloscope measurment of the FPGA ADC noise level at $50 \mathrm{mV}$ offset and $1 \mathrm{~m}$ long cable.

\section{(2.13) Incorporating a Sallen-key Filter into the QTF's Driving Voltage}

While the new shorter cable improved the driving signal, the noise would still correspond to theoretically $60 \mathrm{~nm}$ of displacement, therefore further filtering was required. 
A Discrete Fourier Transform (DFT) analysis revealed the distribution of noise frequencies in the $0-5 \mathrm{kHz}$ band shown in figure 25 (top left) below. While this noise could be low pass filtered to an arbitrarily low level, this would drastically effect the frequency response of the system, noting that it takes many time constants for low pass filters to settle. One solution is to implement a system of two 16-bit drive signals, to achieve an increase in effective resolution, which a similar to a designed used by Rodolfo Fernandez [27]. In this scenario, one channel is aggressively low pass filtered to a corner frequency of roughly 10 $\mathrm{Hz}$, creating a low bandwidth, low noise $\mp 10 \mathrm{~V}$ DC signal. Then, a second higher bandwidth (reduced range) signal is added to this first signal to create a filtered composite output.

The major design goal in this application was not to restrict the MCL's rated closed loop $150 \mathrm{~Hz}$ sinusoidal motion, as such the filters effective time constant was considered. The final filter design happened to be second order. As an approximation, we note that the time for a unit step function to rise from $10 \%$ to $90 \%$ in a single stage $\mathrm{RC}$ network, is proportional to the time constant where,

$$
t_{r} \cong 2.197 \tau
$$

Utilizing a corner frequency of $2.5 \mathrm{~K}$ and time constant about $6 \mathrm{E}-5$ seconds (on the high bandwidth channel), this pertains to a rise time of roughly $1.4 \mathrm{E}-4$ seconds. As a gross approximation, a second order filter with identical stages could be modeled as two cascaded non-interacting systems, in which the equivalent rise time would be,

$$
t_{r(e q)}=\sqrt{2 t_{r}^{2}}
$$


or 2E-4 seconds, several times smaller than the required 6E-3 second period $(150 \mathrm{~Hz})$. Having verified the bandwidth considerations, This second (less aggressively filtered) signal was reduced approximately 100 times, until its noise threshold is below that of the low bandwidth channel. The two channels are finally combined via a summing amplifier to create a single output to drive the MCL.

It was mentioned above that the final filter design was second order. More explicitly, a Sallen-Key Architecture was selected for its simplicity in attaining second order filtering with a single operational amplifier. This eliminates impedance matching required in cascading multiple, single stage filters. The second-order Low-pass filters transfer function $H_{l p}$ (which relates the output voltage to the input) can be expressed as

$$
H_{l p}(f)=-\frac{k}{\left(\frac{f}{F S F \times f_{c}}\right)^{2}+\frac{j f}{Q F S F \times f_{c}}+1}
$$

where $f_{c}$ is the filters cutoff frequency, Q is the quality factor, $F S F$ is the frequency scaling factor, and $\mathrm{k}$ is the DC gain factor [28]. 


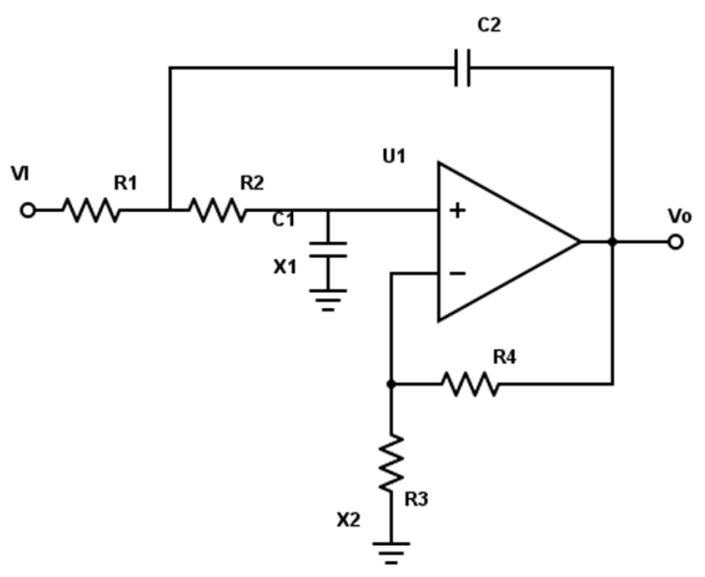

Figure 21 Sallen-Key architecture.

In terms of RC values, the above transfer function requires $K=\frac{R_{3}+R_{4}}{R_{3}}, F S F \times f_{c}=$ $\frac{1}{2 \pi \sqrt{R_{1} R_{2} C_{1} C_{2}}}$, and $Q=\frac{\sqrt{R_{1} R_{2} C_{1} C_{2}}}{R_{1} C_{1}+R_{2} C_{1}+R_{1} C_{2}(1-k)}$ [28]. Since no gain is required in this application, $\mathrm{k}$ can immediately be set to 1 . This architecture lends itself to a variety of filter types such as Butterworth, Bessel, or Chebyshev by appropriate selection of Q and FSF. Because of the fairly uniform distribution of noise around the cutoff frequency, gain flatness in the pass-band was highly desirable. For this reason, a Butterworth filter was selected. As such, the quality factor was set to $\mathrm{Q}=0.707$ and $F S F=1$ [28]. Things can be further simplified by introducing ratio variables $\mathrm{m}$ and $\mathrm{n}$ such that $R_{1}=m R_{2}, R_{2}=$ $R, C_{1}=C, C_{2}=n C$. Using $\mathrm{Q}=0.7071$ (Butterworth), and $\mathrm{k}=1$ in the quality factor equation, the nonlinear ratio of $\mathrm{m}$ an $\mathrm{n}$ can be determined. Under unity gain conditions, this is often chosen such that $\mathrm{n}=3.3$ and $\mathrm{m}=0.229$ [28]. Having determined the parameters for the desired filter characteristics, the corner frequency for channel A was set at $10 \mathrm{~Hz}$, and 
channel B was set to $2.5 \mathrm{kHz}$. Bode plots indicating the theoretical and experimentally measured frequency response is shown below.
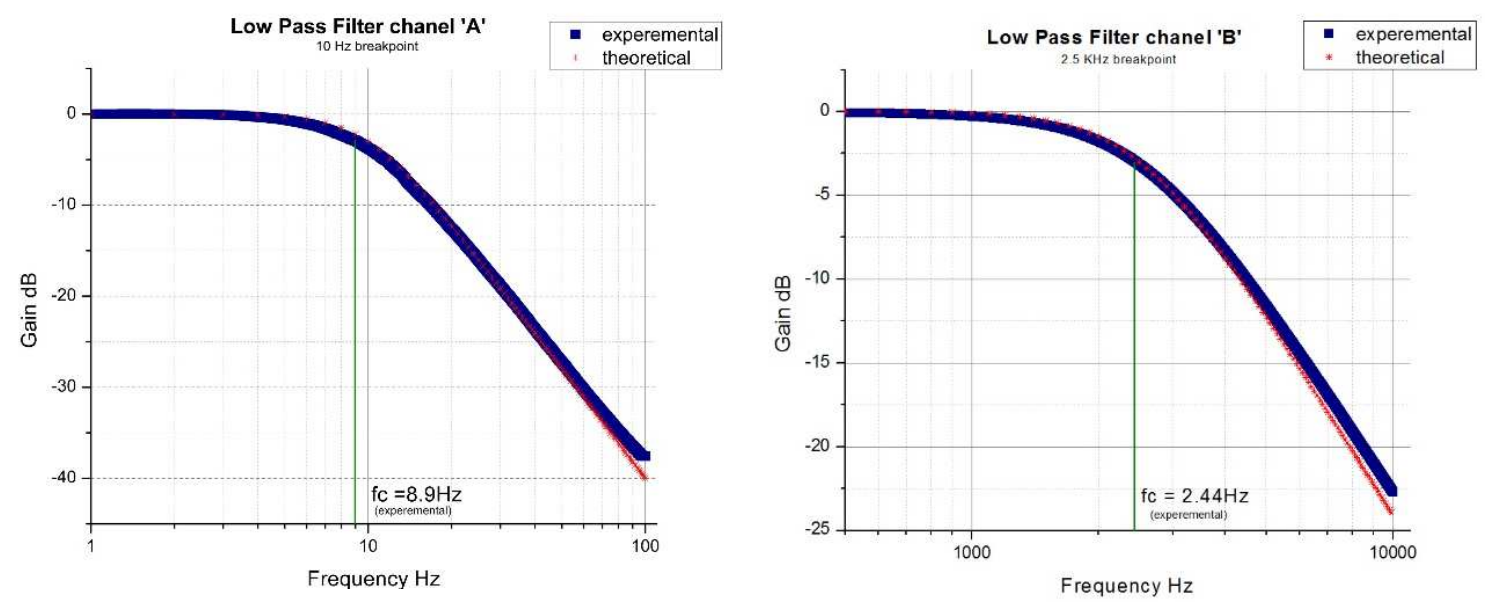

Figure 22 Left) A bode plot of the second order Sallen-key Butterworth low pass filter. Plotted in red is the filters theoretical frequency response with cutoff frequency of $10 \mathrm{~Hz}$. Plotted in blue is the measured frequency response with $-3 \mathrm{~dB}$ point determined to be $8.9 \mathrm{~Hz}$. Right) A bode plot of the second order Sallenkey Butterworth low pass filter. Plotted in red is the filters theoretical frequency response with cutoff frequency of $2.5 \mathrm{KHz}$. Plotted in blue is the measured frequency response with $-3 \mathrm{~dB}$ point determined to be $2.44 \mathrm{KHz}$.

Passing the noisy $24 \mathrm{mV}$ pk-pk drive signal through both channel A and B revealed that the noise level has been attenuated below $4 \mathrm{mV}$ (see figure 23). If needed, an output resistor can be added before the MCL's $1 \mathrm{M} \Omega$ input impedance to further attenuate the noise (and maximum displacement), though it's not ideal as it would contribute additional Johnson noise from the resistor. The filters final design and implementation can be viewed below in figure 24 . 

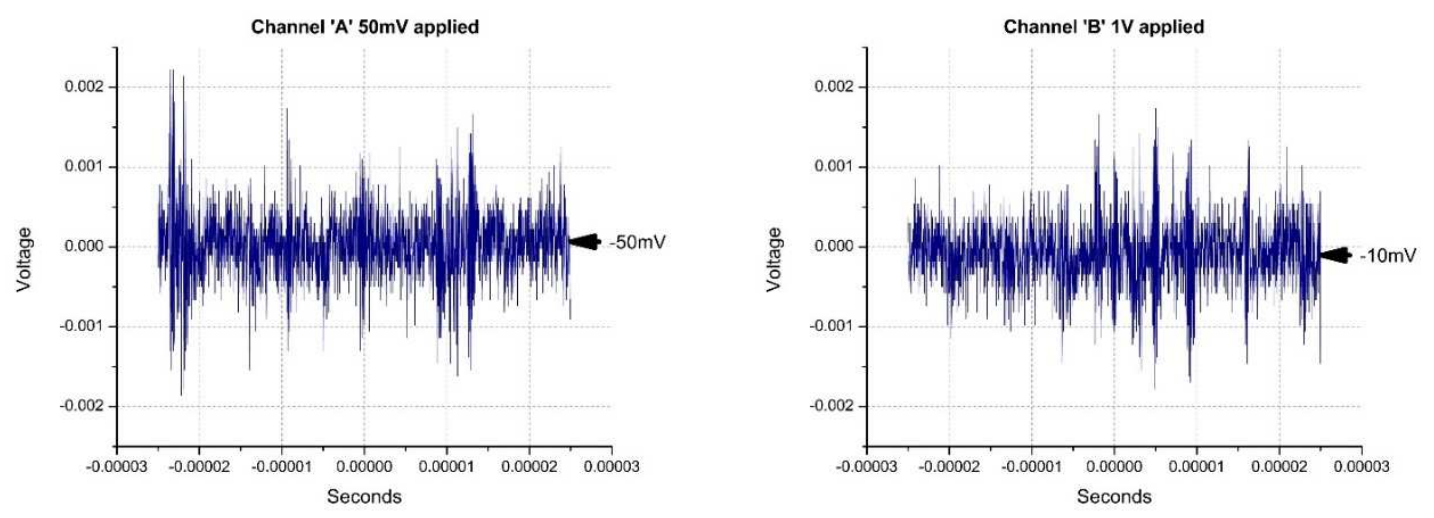

Figure 23 Left) The noise level at the circuit output, after the second order Sallen-key low pass filter (channel A), and summing amplifier, with $50 \mathrm{mV}$ applied to the input of channel A, and channel B grounded. Right) The noise level at the circuit output, after the second order Sallen-key low pass filter (channel B), and summing amplifier, with $1 \mathrm{~V}$ applied to the input of channel $\mathrm{B}$, and channel A grounded.

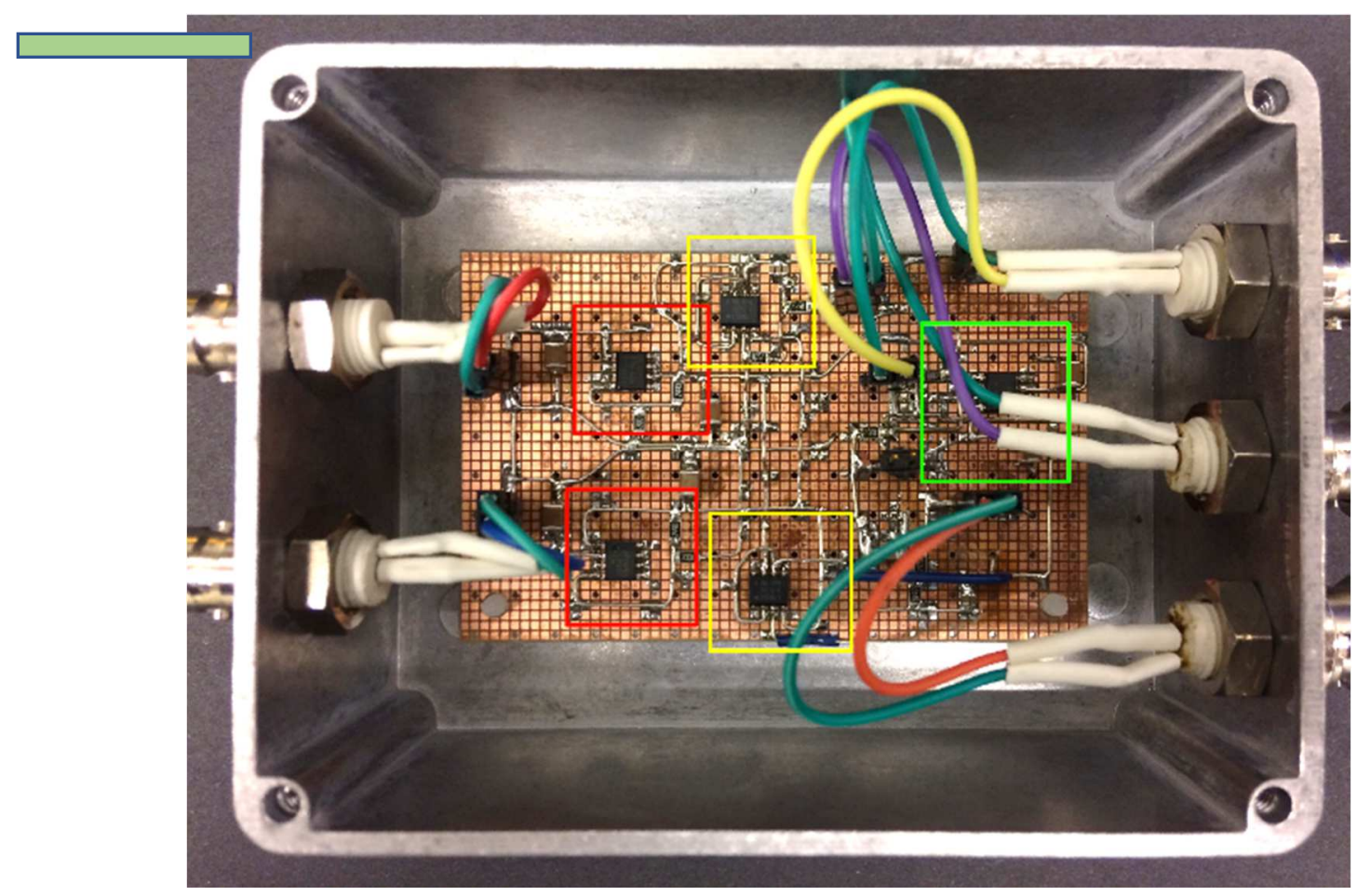




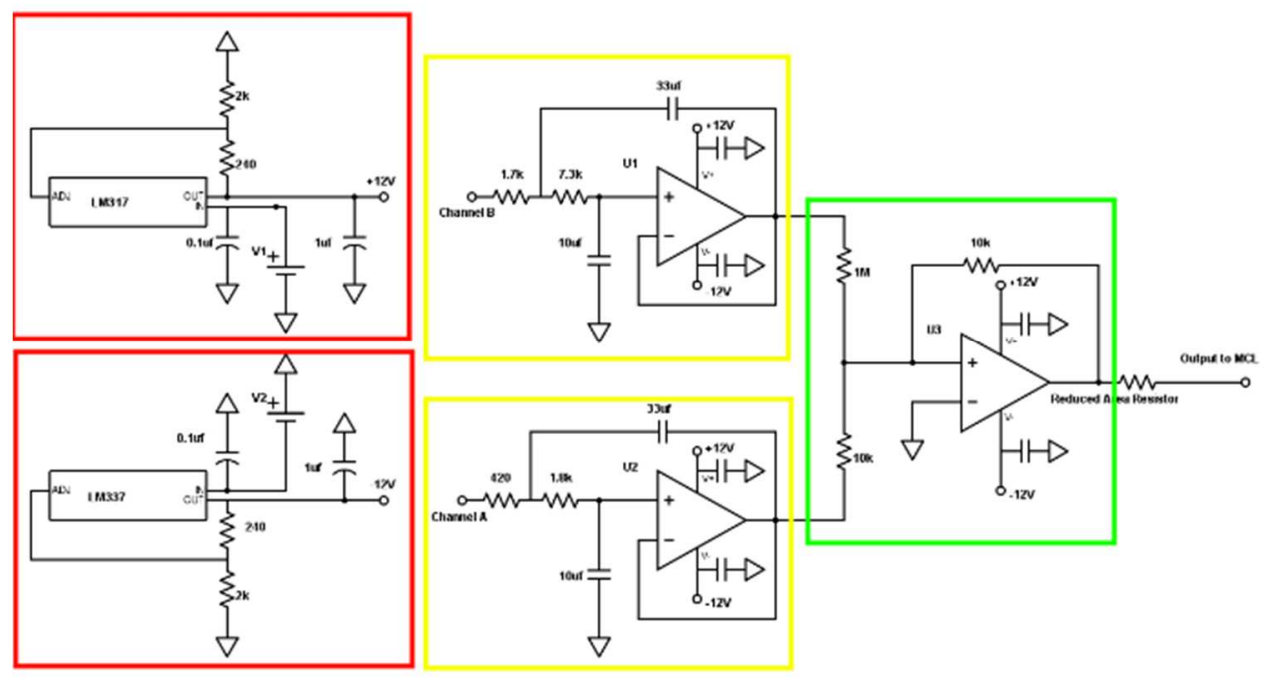

Figure 24 Top) The constructed Filter circuit board with SIO components inside a metal shielded case. The positive and negative regulators outlined in red, the two second order Sallen-Key filters in yellow, and the summing amplifier outlined in green (green scale bar $\sim 4 \mathrm{~cm}$ ). Bottom The schematic circuit diagram illustrating the positive and negative regulators outlined in red, the two second order Sallen-Key filters in yellow, and the summing amplifier outlined in green.

A plot showing an increase in noise performance after the final composite filter was fully implemented is displayed in figure 25. The quantile-quantile (QQ) plot in figure 25 top right compares FPGA noise distribution against a standardized Gaussian distribution, and reveals that the unfiltered drive signal directly from the FPGA is colored (as evident in the QQ plot tails deviating from the red dashed line). However, after filtering the noise level is significantly lower, and appears to more Gaussian apart from a power supply peak at $60 \mathrm{~Hz}$ (and its harmonics) which can be eliminated by powering with a battery. 

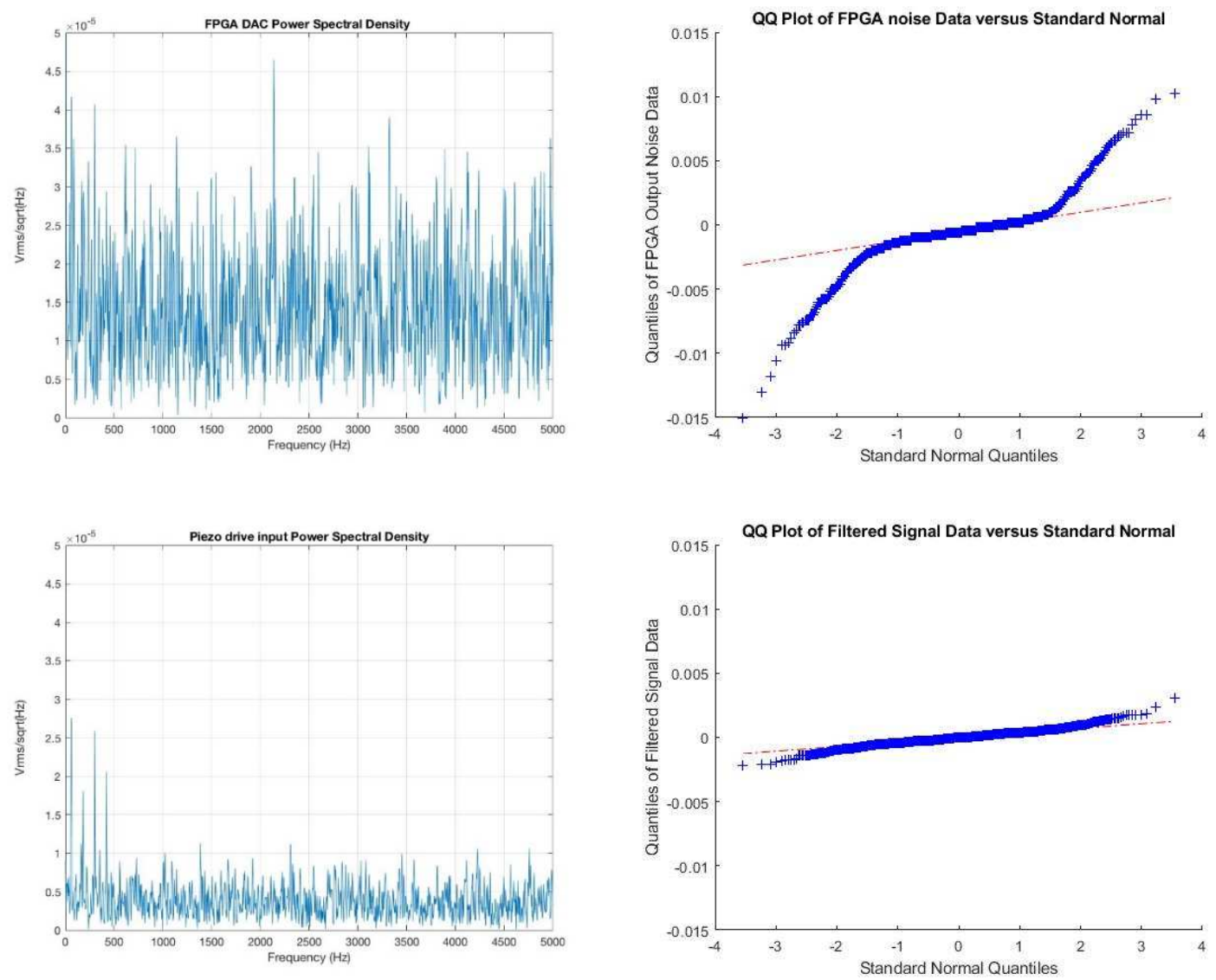

Figure 25 Top Leftt) Power spectral density plot of the FPGA noise before filter. Top Right) QQ plot comparing the FPGA noise distribution against a standardised gaussian distribution. Bottom Left) Power spectral density plot of Filtered drive signal. Bottom Right) QQ plot of filtered drive signal compared to stardardised gaussian distribution.

\section{(2.14) Operating a Strain Gauge Differential Amplifier}

The MCL Piezo stage is driven in closed look feedback, meaning the stages output will follow the calibrated drive signal provided by the course and fine channels described in the previous section. However, instead of relying on the drive signal to infer the displacement of the stage, physical measurement is performed using the factory integrated strain gauge sensor. This is preferred because it more accurately represents the stages 
displacement after the closed loop control. The standard strain gauge output generates an analog $0 \mathrm{~V}$ to $10 \mathrm{~V}$ signal corresponding to the 50 -um total stage displacement. Considering the FPGA's 16-bit analog to digital converter, this output range yields a maximum theoretical resolution of about $1.5 \mathrm{~nm}$. This value does not take into consideration any induced or ADC noise which would further degrade the resolution. One simple method for increasing this strain gauge resolution would be to amplify the original signal at the source. However, the output range is already at the $10 \mathrm{~V}$ rated maximum input on the FPGA. To solve this problem, a differential amplifier was designed and implemented. The amplifier was constructed using an INA217 instrumentation op amp to amplify a difference in input signals between the strain gauge, and a second filtered analog reference input supplied by the FPGA. This reference was configured such that the resulting amplified output never exceeded the 10 V FPGA input limit. Correct scaling was done in LabVIEW by splitting the MCL course channel into 10 equal regions. While the course drive signal is positioned within the $n^{\text {th }}$ region (where $\mathrm{n}$ is an integer between 1 and 10), the Strain gauge sensor will clearly produce a signal between $\mathrm{n}$ and (n-1) V. knowing this, a filtered reference signal is produced by the FPGA at $\mathrm{n}$ volts. This windowing ensures the voltage difference between the strain gauge output and reference always remains within $1 \mathrm{~V}$. Using a differential gain of 10 , the new strain gauge signal is then properly scaled to the $10 \mathrm{~V}$ input limit. In this way, the differential strain gauge output will oscillate between $0 \mathrm{~V}$ and $10 \mathrm{~V}$ in a saw tooth fashion each time the drive signal extends the course channel into the neighboring region. In the end, this differential amplifier improves the theoretical resolution from $1.5 \mathrm{~nm}$, to 1.5 Angstroms. To record an absolute position rather than a 
relative measurement, $\mathrm{n}^{*} 2^{15}$ bits $(\mathrm{n} * 10 \mathrm{~V})$ is analytically added to the diff strain gauge output in LabVIEW. In implementing this design, the circuit itself was constructed following the schematic diagram of figure 26 right) using one sided surface mount proto board with BNC headers directly soldered to the board. to minimize noise, the circuit board was placed inside of a recycled Radio Frequency (RF) shielded can, which can be seen in figure 26 left). With the nanopositioner's low noise drive signal provided by composite filter, and this high-resolution strain gauge amplifier, the user is afforded precise control of the probes relative position.

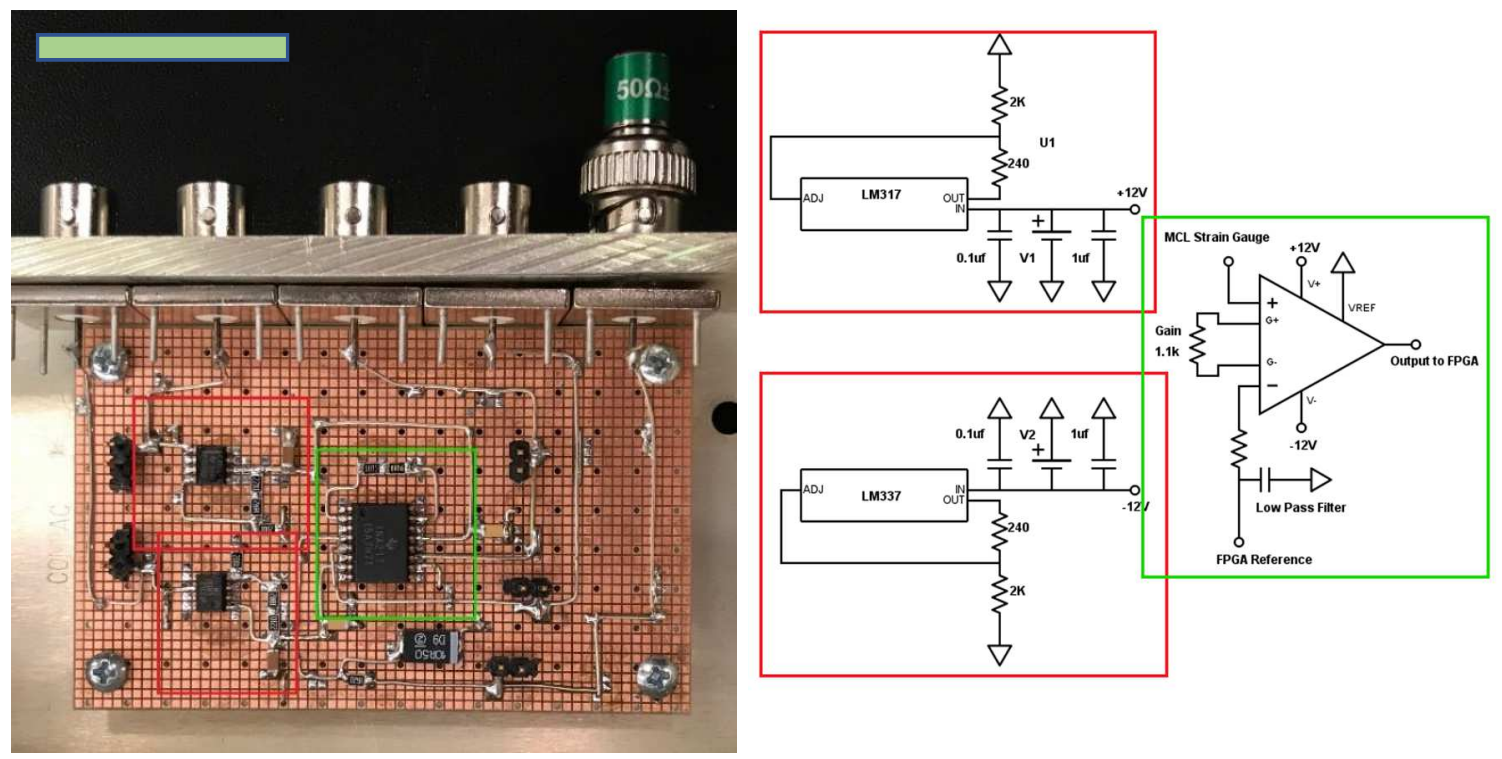

Figure 26 Left) The constructed SIO differential amplifier circuit constructed on proto-board, placed inside a recycled RF can, (green scale bar $\sim 4 \mathrm{~cm}$ ). Right) Schematic diagram displaying the simplified low differential amplifier and low pass filter circuitry in red, with bipolar voltage regulation in red. Note the figure is displayed in a block diagram for clarity. The $12 \mathrm{~V}$ output from the voltage regulators in red are physically connected to the $12 \mathrm{~V}$ input to the op amp outlined in green. 


\section{(2.15) Construction of a Humidity Chamber with Added Salts to Establish Fixed Points of Humidity Levels}

As the primary focus of this microscope is to probe the mesoscopic fluid layer that is present on many substrates in ambient conditions, it is important to understand when a fluid layer of appreciable size will be present. One topic that we investigate is the formation of a meniscus water bridge that connects the probe and substrate. This meniscus formation is highly dependent on the wetting properties of the probe and substrate, as well as the humidity and temperature of the surrounding environment. The details of this inquiry will be presented below in chapter 3 , here we concern ourselves with creating an environment that is conducive to a reliable meniscus formation. Simply, a water meniscus may suddenly form when the probe's apex is placed in the proximity of a substrate with sufficient water layer. The presence of this water layer is related to relative humidity [29]. Therefore, to increase the probability of water meniscus formation, an acrylic chamber was built to enclose the microscope head-stage, and establish a stable relative humidity $(\mathrm{RH})$ above the typical 35\% existent in our lab ambient. The RH is established through a combination of two methods, 1) injecting low pressure humidified air, and 2) placing inside the chamber a saturated salt solution. A Kestrel 4200 flow tracker monitors the humidity in real time. The low-pressure humidified air was created by first sending compressed air (regulated at 0.5 psi) through a tubing network, then through a humidifier (see below), and finally the vapor stream is injected into the chamber through an open cell foam diffuser. The humidifier comprises a stoppered 1L Erlenmeyer flask containing deionized water and two tubes, one bubbling air through a ceramic stone and the other returning the humidified air to the 
chamber. On the other hand, a saturated sodium bromide solution was placed in a shallow watch glass inside the chamber to exploit its fixed-point humidity of $59.1 \%$ at $20{ }^{\circ} \mathrm{C}$ [30]. Through experimentation, it was found that employing both methods concurrently allows the user to rapidly bring the chamber to the dictated relative humidity, while still exploiting the stability provided by the saturated salt solution. A model of the acrylic chamber, and schematic diagram of its humidifier operation is presented below in figure 27.
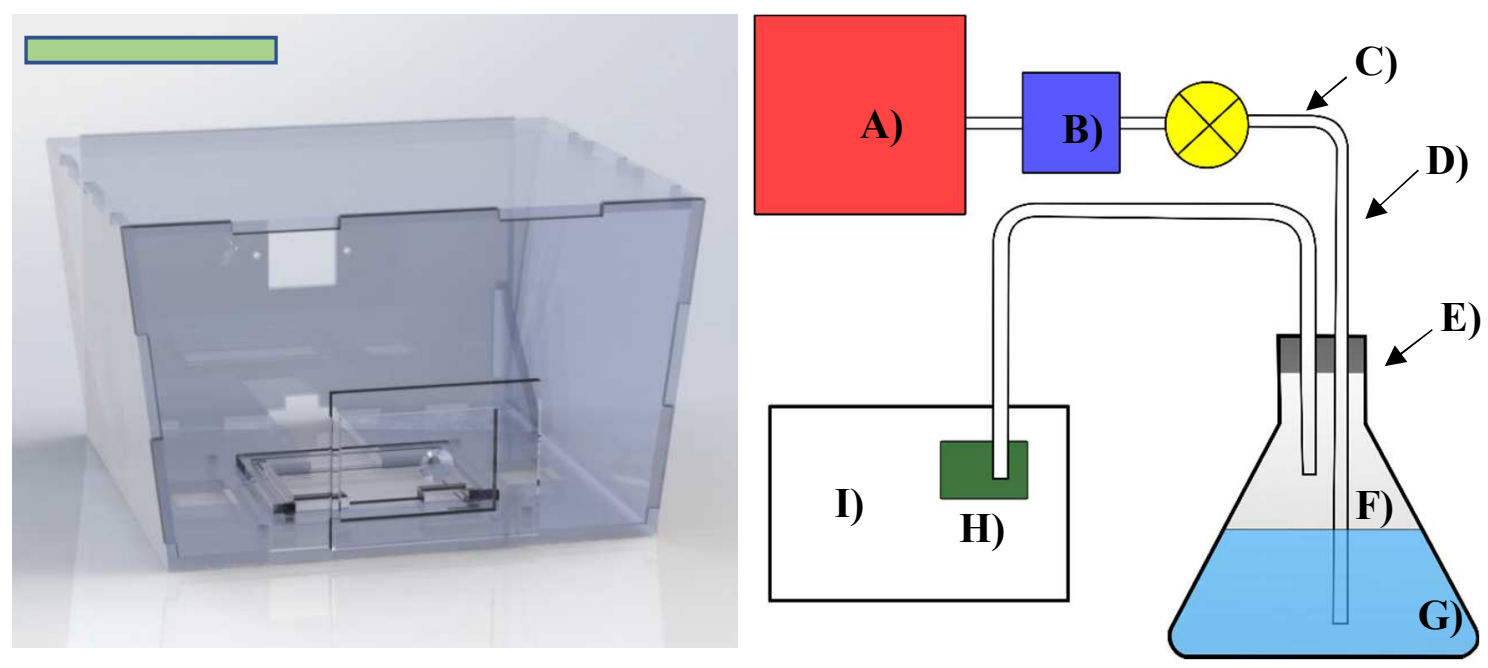

Figure 27 Left) Rendering of Acrylic humidity chamber, designed in solid Works and manufactured using a laser cutter and Dichloromethane cement. Chamber can easily maintain relative humilities in excess of $80 \%$, (green scale bear $\sim 10 \mathrm{~cm}$ ). Right) A) External air compressor, B) Pressure regulator, C) Ball valve, D) Tubing to direct humid air, E) Rubber stopper, F) Erlenmeyer flask, G) Water, H) Open cell foam diffuser, I) Humidity chamber.

\section{(2.16) Use of Stepper Motors to Control the Probes Coarse Approach}

To best utilize the new humidity chamber, stepper motors were added to drive the micro positioners on all three legs of the SPM head. This modification means that once the tuning fork sensor is installed, the humidity chamber can be closed, and the system brought to equilibrium without ever needing to open the chamber for the duration of the experiment, 
thereby not disturbing the steady state conditions. In the past, the chamber must have been opened to access the micro-positioning screws each time a coarse adjustment in position was required. The stepper motors simply allow this process to be done remotely. One problem encountered while installing the stepper motors, was coupling the drive shafts between the motor and the micro positioning screw. as the threaded shaft rotates in and out, the distance between the end of the stepper motor drive shaft and threaded shaft changes. To solve this problem, an expanding drive coupler was designed by Alex Challey, and Rodolfo Fernandez, which consists of nested cylinders and keyed shafts allowing both to rotate concentrically, while maintaining freedom in the axial direction. An exploded diagram of the drive coupler can be seen in figure 28 left.
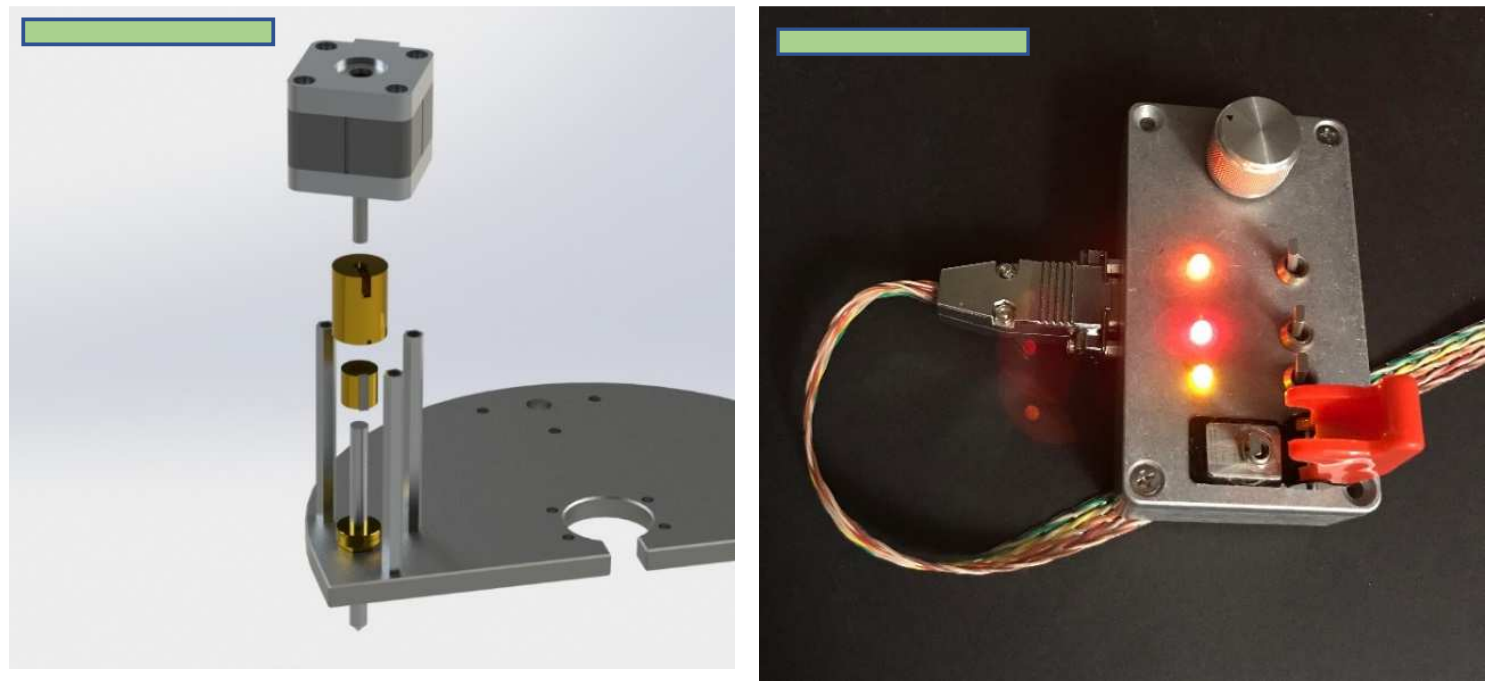

Figure 28 Left) Exploded diagram displaying the specially designed drive coupler design linking the stepper motor and threaded positioner shaft. The two brass cylinders' nest inside each other with keyed slots aligning to allow compliance in the axial direction, (green scale bar $\sim 8 \mathrm{~cm}$ ). Right) Stepper motor controller including optical encoder knob to manipulate shaft rotation, 3 switches (with corresponding LED indicators) to select the desired arrangement of stepper motors, and switch (below safety) to toggle between high speed and fine stepping, (green scale bar $\sim 6 \mathrm{~cm}$ ). 
Each stepper motor is driven by an independent stepper motor control board Purchased at Spark fun (product code: RB-Spa-888), and powered using a large 24V linear supply from International Power (IHBB15-1.5), and can be selected independently or as a group by enabling the corresponding rocker switch (on controller pictured in figure 28 right) with LED indicator verification (yellow for left, Orange for right, and Red for rear). The stepping control is provided using an optical encoder knob, passed to an Arduino Uno microcontroller through an analog low pass filter (to reduce switch bounce). Digital signal processing inside the Arduino determines the encoder direction (counter clockwise to approach, or clockwise to retract) and number of cycles. The large safety switch to the left side of the controller is used to toggle between $1 / 4$ steps increments (for fast approach) or (1/16) steps increment for fine approach. It should be noted that while the different step size steps correspond to the different angular rotation speed at the shaft, only an integer number of steps will be taken per cycle. This is because when the stepper motor is powered on after being left 'mid step' the shaft can abruptly align with the nearest integer step, thereby possibly crashing a probe when in close proximity to a substrate.

\section{(2.17) Configuration for Measuring Probe-Substrate Tunneling Current}

This SPM allows for several different tunneling configurations depending on the specific needs of the experiment. However, each case requires a robust electrical connection between the conductive probe, and the larger tunneling circuitry. Most often, the conductive tapered probe is connected directly the aluminum electrode present on the quartz tuning forks from the factory. It is important to attach the probe to the electrical pad 
that is connected to the tuning forks driving potential, as opposed to the other lead connected to the lock in. This is to ensure a low output impedance, and correct tuning fork electrical amplitude measurement. In the past, the aluminum electrodes extended to nearly the end of the tuning fork tine, meaning the conductive probe came into direct contact while epoxying. Though, in recent years, QTF's have been purchased where the electrodes have only come within 70 um or so of the tines edge. This poses a problem because while it is possible to increase the probes length to make the connection directly, it is undesirables as the increased weigh diminished the quality factor, and shifts the resonant frequency of the probe out of the optimal frequency band. One solution that has proven somewhat successful, is completing the electrical connection (of standard short length probes) with a small drop of silver paint applied with tweezers under a microscope. Having made the low impedance connection to the probe, the next task is making an electrical connection to the sample. Often a conductive sample such as Highly Ordered Pyrolytic Graphite (HOPG) or a metallic sputtered sample is used. This sample is adhered to a $1 \mathrm{~cm} \times 1 \mathrm{~cm}$ cleaved square of Si using double sided carbon take. Finally, a piece of magnet wire (with the enameled end removed), is placed on the carbon tape near the sample and electrically connected using silver paint. At this point, all that remains is to electrically connect the substrate to an amplifier that will be used to measure the tunneling current. The simplest scenario is attaching the sample to a Stanford Research 570 current amplifier. This device has a low impedance input to a virtual ground, as well as an adjustable input bias use to set the tunneling potential. Biasing the sample is more desirable than adding a DC bias to the tuning fork as the additional bias could affect the piezoelectric driving characteristics. The 
current amplifiers output can be fed directly into the FPGA to measure the DC current contributions. However, because the probe is also connected to the AC driving potential, it is also possible to measure $\mathrm{AC}$ tunneling current contributions by first passing the amplified SR570 output into a Lock in. A schematic diagram illustrating the building block connections in the tunneling circuit (as well as others) can be seen below in figure 29 .

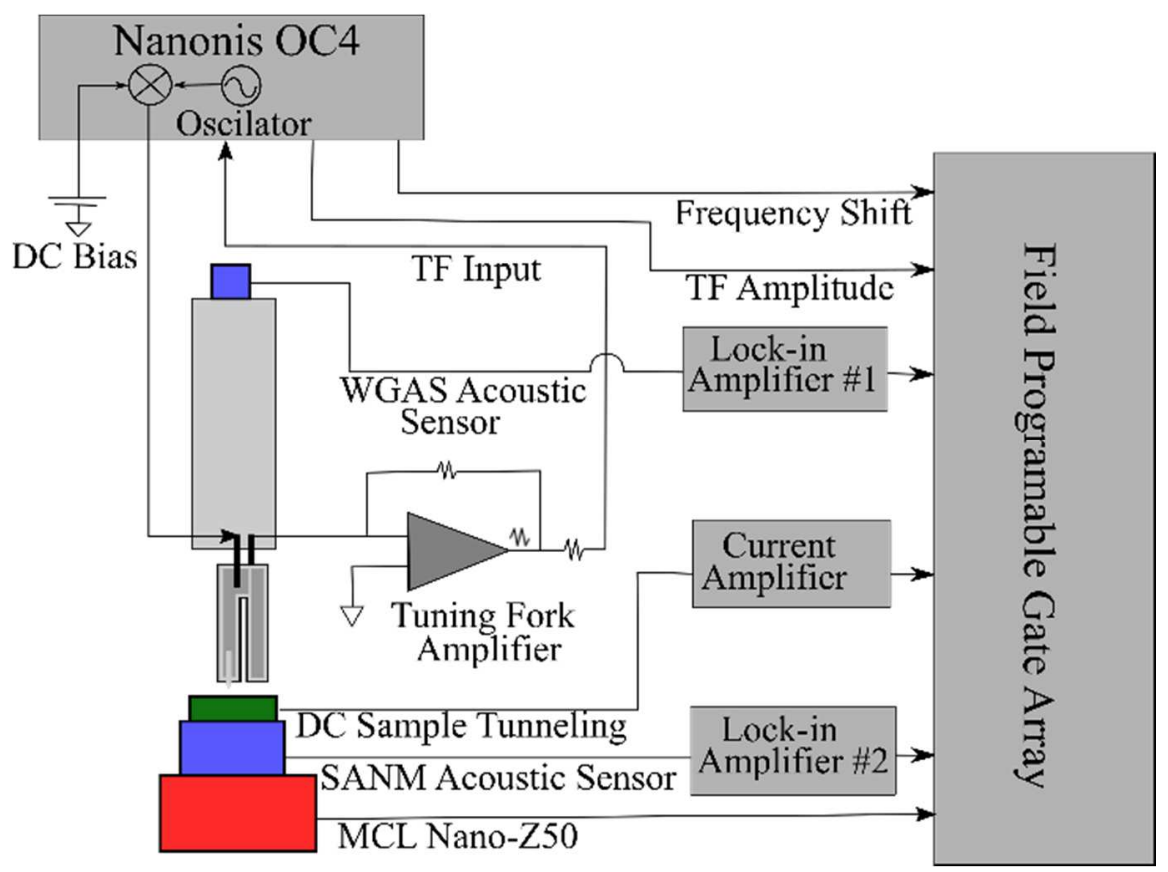

Figure 29 A schematic showing the electrical connection present in the tunneling current circuitry (measuring DC current contributions here) The probe can either be biased at the tuning fork by adding a DC potential to the ADD input of the OC4, or directly at the current amplifiers input.

\section{(2.18) Sensors' Electrical Noise Considerations}

In building microscopy systems, electrical noise and cross talk must continually be managed. Because the SANM sensor generates such a small electrical response (typically on the order of a couple Nano amps or less) the lock-in amplifier driving the sensors must be relatively high gain, making them more susceptible to electrical noise. An example of 
this noise is displayed below in figure 30. This noisy spectrum in (figure 30 left) was recorded while Characterizing the tuning fork and acoustic sensors in preparation for an experiment on March 15 2018. In this particular series of experiments, a tuning fork with additional mass was required. The additional mass red-shifted the resonance frequency about $1200 \mathrm{~Hz}$ (with respect to a bare QTF) into a frequency band that is not often utilized. A large SANM resonant peak appeared at about $31.3 \mathrm{kHz}$, while the QTF and WGAS showed the tuning fork resonating closer to 31500 . In looking at this spectrum one point of interest is that fact that the tuning fork trace displayed a 'beat' at the resonant frequency of the SANM sensor. This was noise originally interpreted as the SANM sensor picking up a mechanical resonant mode in the cavity which in response was mechanically picked up by the tuning fork sensor. However, one month later, on April 06 2018, a subsequent spectrum was taken (figure 30 right), with a different tuning fork wherein the resonance peak of all three signals coincided with the SANM's resonant frequency found earlier. If this was truly a mechanical resonance mode in the microscope being picked up by the SANM sensor (as previously interpreted), the SANM magnitude should have been much larger when the QTF resonance coincided with the SANM peak than then case when the QTF's peak was shifted off the SANM peak. This was not observed, in fact magnitude of the SANM peak was the same in both instances. 


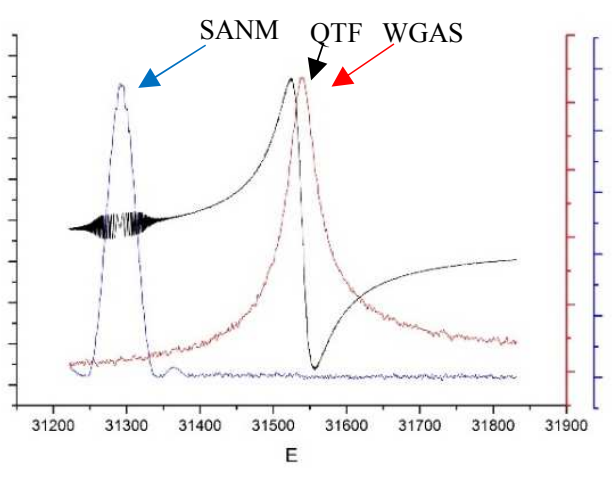

Frequency (Hz)

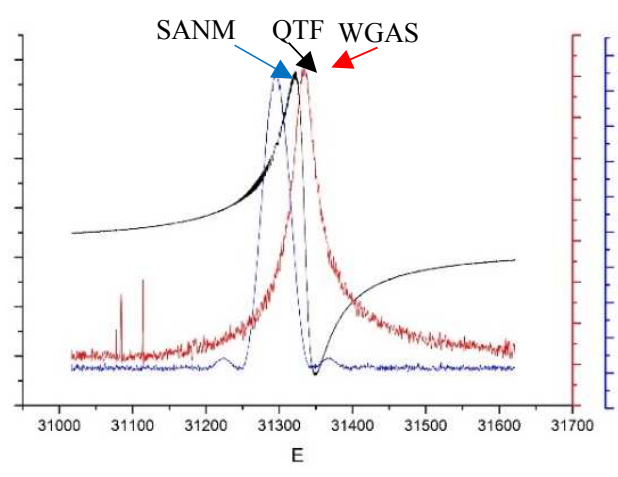

Frequency $(\mathrm{Hz})$

Figure 30 Left) Spectrum taken while characterizing the tuning fork for an experiment on 2018-03-15. Notice SANM peaks near $31.3 \mathrm{kHz}$, while QTF, and WGAS peak between 31.5-31.6 kHz. Right) Spectrum taken while characterizing the tuning fork for experiment on 2018-04-06. Notice SANM again peaks near 31.3K, while QTF and WGAS peak slightly above.

To further investigate the source of this spurious peak at $31.3 \mathrm{kHz}$, the SANM acoustic sensor was removed and replaced with an unshielded section of copper wire acting as an antenna. In this case, even without the piezoelectric acoustic sensor connected, a resonant peak was detected on the lock-in amplifier at precisely the same $31.3 \mathrm{kHz}$ frequency. This of course showed that the signal was not a mechanical resonance, but instead electrical crosstalk being picked up by an external source. With assistance from Leroy Laush, the noise source was identified as coming from the Cathode Ray Tube (CRT) display located on the front panel of the SR850 lock in amplifier. Figure 31 left) displays a waveform of the noise captured with the Tektronix digital storage oscilloscope, using a bare wire as an antenna located a few $\mathrm{cm}$ away from the CRT. Note the $80 \mathrm{mv}$ signal magnitude measured directly without any kind of amplification! Figure 31 right) displays the waveforms power spectral density. This result confirms that the spurious SANM resonance peak measured in figure 28 located at $31.3 \mathrm{kHz}$, is actually the source of crosstalk from the second harmonic of the CRT raster scanning. The cross talk was finally 
mitigated by improving cable shielding, and physically placing the SR850 lock-in amplifier as far from the lock-in monitoring the SANM as possible.
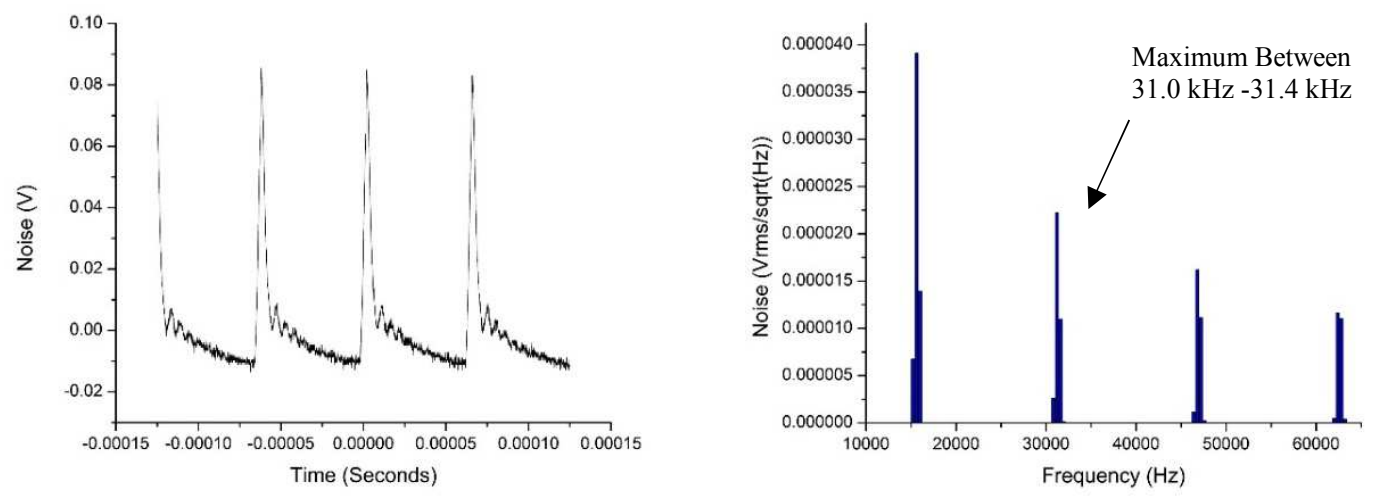

Figure 31 Left) Signal trace captured in the time domain using a Tektronix digital storage oscilloscope and computer interface showing induced noise in an antenna probe placed near the Cathode Ray Tube (CRT) display on the SR850 lock in. Right) Power spectral density plot of the same CRT noise displayed left. Note the second harmonic peak is located between $31.0 \mathrm{kHz}$ and $31.4 \mathrm{kHz}$. 


\section{Chapter 3}

\section{Results}

\section{(3.1) Interactions with the Adsorbed Water Layer}

The source of viscoelastic interactions at the atomic and mesoscopic scales is not well understood. Comparing experimental sheer force measurements, against the theoretical contributions due to Coulomb drag, Joule heating, or black-body fluctuations; Karrai et al were forced to postulate that the probe-substrate interaction was likely due to the presence of a third body (presumably composed of water and hydrocarbons) filling the gap between the substrate and probe [17]. This theory is supported by an earlier paper published in Applied Physics Letters by M. Luna, J. Colchero, and A. M. Baró, wherein the research group used a Scanning Force Microscope to show that the oscillation amplitude of their cantilever probe, began to decrease (due to dissipative interactions) with more than $6 \mathrm{~nm}$ separation between the probes apex and substrate (for a given humidity), a larger separation distance than where coulombic interactions should dominate [31]. Luna et al, attributed the dissipative interactions to a water layer which is present in atmospheric conditions and adsorbed on both the substrate and probe. In this way, as the probe approaches the substrate, a meniscus or 'water bridge' may spontaneously form, connecting the adsorbed water layers on the probe and substrate. This type of wetting phenomena has been studied extensively, including by Jacob Israelachvili who states "Liquids that wet or have small contact angles on surfaces will spontaneously condense from vapor into cracks and pores as bulk liquids" (p.330) [32]. R. Digilov, another researcher analytically investigating the spontaneous capillary formation between Nano 
scale features further states "A liquid bridge coexists in stable equilibrium with the surrounding vapor and is separated from the vapor by a concave meniscus of mean curvature defined by the Kelvin equation [this radius is Referred to as the Kelvin radius]" [33]. The Kelvin radius can be calculated as

$$
r_{k}=\frac{\gamma V}{R T \log \left(\frac{p}{P_{S}}\right)}
$$

where $\gamma$ is the surface tension of the fluid, $V$ is the molar volume, $\mathrm{R}$ is the universal gas constant, $\mathrm{T}$ is the temperature, and $\left(P_{S} / P_{S}\right)$ is the relative humidity [32]. Evidently, as the relative humidity increases, so too does the Kelvin Radius. Luna et all recorded various approach retraction curves with their AFM microscope at different humidity's, and found that the amplitude of oscillation begins to decrease when the separation distance between the probe and substrate is roughly twice the Kelvin Radius (at least for humidity's that aren't very large or small) giving credence to the dissipative meniscus model [31]. The possibility of this meniscus water-bridge formation, and viscus third body will be analyzed below.

\section{(3.2) A Note on Surface Hydrophobicity and Sample Preparation}

It has been demonstrated [34], that the relative hydrophobicity between a probe and substrate, can lead to measureable effect on the viscous friction force, as well as meniscus formation. In the Physical Review Letters publication 'Viscous Water Meniscus under Nano-confinement', Major et al show that when both the probe and substrate are made Hydrophobic (via deposition coating with $\mathrm{CH}_{3}$ ), the viscus friction force begins to increase, only once the adsorbed water layers on the probe and substrate make 'film-film' contact. 
In this case, a meniscus is unlikely to form (as verified through numerical simulation), and corroborated by fact that the tip-substrate separation distance corresponding to the onset of friction forces, is independent relative humidity [34]. However, when both the probe and substrate are made hydrophilic (via deposition with $\mathrm{COOH}$ ), the viscus interaction first experiences a sharp increase in friction force (attributed to a meniscus formation). As the approach continues, the viscus interaction reaches a maximum before ultimately decreasing after the adsorbed water is 'squeezed' from the gap confining the liquid (in high humidity cases), until the eventual 'film-film' contact, whereby the viscous interaction again increases as usual [34].

In the results presented here, Hydrophobic silicon substrates are prepared via etching in a dilute hydrofluoric acid solution. Hydrofluoric acid is extremely dangerous, and exposure may be fatal. As such, users should be properly trained, and wear adequate personal protective equipment including: Goggles, face shield, acid resistant apron, long pants/sleeves, appropriate shoes, and thick 10-20 mil neoprene gloves [35]. The dilute etch solution is prepared $500 \mathrm{ml}$ at a time, as needed from the concentrated acid. In this process, $480 \mathrm{ml}$ of deionized water is placed into a proper 11 Nalgene chemical storage container. Then, $20 \mathrm{ml}$ of concentrated (49\%) is extracted using a pipette, and added to the water, creating a $2 \%$ dilute HF solution. Having created the dilute solution, the Si etching process begins by pouring the dilute HF solution into a shallow, non-reactive plastic container. 1 cm square sections of silicon are then placed (submerged) into the solution using plastic tweezers, and allowed to etch for $2 \mathrm{~min}$. Finally, the etched Si samples are extracted, rinsed thoroughly with deionized water, and dried with compressed nitrogen. In the process, the 
HF etch solution renders an Si interface terminated with single layers of Hydrogen bonds (which begins to oxidize within minutes) [36]. This H-terminated structure has low polarization, thereby promoting any water present in the surface to preferentially hydrogen bond with itself, yielding a large (hydrophobic) contact angle.

Conversely, hydrophilic substrates in this thesis were prepared using a Piranha etch solution. This process began by cleaving a $1 \mathrm{~cm}$ square section of silicon wafer (with its native oxide intact). The substrate is then ultrasonically cleaned in a solvent bath of acetone for 15 minutes to remove particulates and oils from the surface, before being rinsed thoroughly in deionized water. Next, the etch solution is prepared, by combining three parts of concentrated sulfuric acid and one part of $9.8 \mathrm{M}$ hydrogen peroxide. It is worth noting that care must be taken when adding the hydrogen peroxide to the sulfuric acid; it must be done drop by drop, as the reaction is extremely exothermic. With the Piranha solution prepared, the silicon wafers are lowered into the etch solution and allowed to react for 30 minutes. Finally, the sample are removed and rinsed with deionized water, where a hydrophilic $<20^{\circ}$ water contact angle is verified. This Piranha cleaning procedure should render an $\mathrm{OH}$ terminated interface on the silicon substrate [37]. The hydroxide termination allows hydrogen bonding between the surface and water which produces the small contact angle.

Of course, when a fluid is confined between the probe and substrate, the probes hydrophobicity plays just as important a role as the substrate. Through direct measurement of contact angles, it is observed that typical etched gold probes are neither highly hydrophobic, or highly hydrophilic; rather the relative hydrophobicity is determined by 
probe cleanliness (contamination from sustained atmospheric exposure leads to increased contact angles). In this way, it is important to understand how the relative hydrophobicity of surface-probe system can affect the likelihood of a robust meniscus formation. This meniscus formation may play an important role when investigating topics such as the source of probe plastic deformation, or acoustic emission.

\section{(3.3) Monitoring Probe Deformation. Case of Tapered Gold Probes:}

The goal of this study is to investigate the viscoelastic properties of fluid confined between two solid, trapping boundaries comprising: $i$ ) a stationary flat substrate, and $i i$ ) the apex of a laterally oscillating tapered probe. This investigation includes inferring the fluid's elastic and damping coefficients (based on observations of the mechanical response of the laterally oscillating probe), in addition to monitoring the acoustic emission from the fluid subjected to shear oscillatory stress. To ensure accurate and reliable measurements of these properties, it is desirable to understand the robustness of the tapered probe against compliance, and an eventual plastic deformation. The tapered morphology of the probe is attained via a chemical etching method descried previously in section 2.2. The robustness of etched gold probes is first evaluated. Gold is an ideal material choice for this specific experiment because, given its relatively low yield strength, any contact between the probe

and solid substrate should result in deformation of the probes apex. This eventual deformation can be quantified by post examination under a Scanning Electron Microscope (SEM). The pursuit of these tests is triggered by prior observations in our laboratory revealing an elastic response of the probe upon its interaction with the substrate and its 
adsorbed water layer (its resonance frequency increases as the probe-substrate distance decreases) [38]. We pursue verification that this elastic effect on the probe is not due to mechanical contact between the probe and the solid substrate but instead the mediating third body (fluid). Tests of the probe robustness (as preformed here) provides then reliable means to measure the fluid properties, by discarding mechanical-mechanical contact as a potential source of the elastic effects on the probe's lateral oscillations motion. A protocol was designed to verify these postulates. A laterally oscillating tapered gold probe approaches a substrate until a given dissipative interaction force (selected previously by the user) was measured, at which point, the probe is retracted and be re-characterized with the SEM to evaluate any potential deformation of probe's apex. This process was repeated several times at increasingly larger interaction forces to evaluate the point at which the probe has essentially" pushed through" the fluid layer, and contacted the solid substrate (resulting in visible deformation). The detailed experimental process is described below.

The tapered gold probes were manufactured using the process outlined in Section 2.2 above. The probe's topography was evaluated using a modified Hitachi SEM (model S-4160) to store digital images of the probes profile for later comparison. Next, the tuning fork probe was installed into the SPM head wherein the mechanical displacement is calibrated (as described previously in section 2.4), and the sensitivity of the acoustic transducers are optimized (both WGAS and SANM). The humidity chamber (containing also a Sodium Bromide solution inside) is then installed (with humidifier hardware turned on), at which point the system is left for approximately 30 minutes for humidity and thermal characteristics to reach equilibrium (as verified using a digital humidity sensor (Kestrel 
4200 Flow Tracker)). Next, the microscope head is coarsely lowered using the 3 stepper motors until the probes apex is within a distance of about 10 microns from the substrate, which is verified using an USB endoscope chamber camera (Andonstar 500x USB microscope). At this point, fine tilt adjustments in the microscope are made (by independently adjusting the 3 micro positioners which comprise the tripod legs on the SPM head) to ensure the long axis of the tuning fork (and probe) are positioned orthogonal to the sample's surface. This placement reduces the possibility that the probe will "tap" the sample at an angle, while simultaneously ensuring the probes apex is the first point of contact between the probe and sample. Having coarsely positioned the microscope head, a fast approach begins by raising the MCL piezo positioner (to which the SANM acoustic sensor and sample are attached), toward the QTF probe at a rate of $10 \mathrm{~nm} / \mathrm{second}$. The automated approach system continuously monitors the probe's instantaneous amplitude, and compares it to a previously selected 'set point' whose value is initially chosen 3 times below the noise level. At some instance during this 'fast approach', the probe's apex (with adsorbed water layer) will begin interacting with a mesoscopic fluid typically found adsorbed onto the substrate (possibly through the spontaneous formation of a meniscus water bridge). Dissipative components of this interaction will cause the QTFs' oscillation amplitude (measured in frequency modulation) to slightly decrease. If the amplitude falls below the 'set point', the piezo stage is immediately retracted a few hundred nanometers. A National Instruments FPGA (PCIe-7852R) utilizes in this this instrument for both data acquisition and control. It comprises an onboard $40 \mathrm{MHz}$ clock, and can perform $750 \mathrm{k}$ samples per second on each analog channel. Meaning, the systems response time will be 
limited by the time constant of the lock in amplifier monitoring the tuning fork oscillations. In this case, the QTF's lock in bandwidth is $389 \mathrm{~Hz}$ (or 400 us). Considering that an abrupt change in the QTF's amplitude may take as many as 5 time constants to be reflected in the output signal, this would correspond to a $2 \mathrm{~ms}$ reaction time. Because the 'fast' approach rate is $10 \mathrm{~nm} / \mathrm{s}$, this reaction time would correspond in 0.2 Angstroms of travel (beyond the point where the amplitude first decreased below the 'set point'), a distance unlikely to result in contact between the probe and solid substrate. Having quickly retracted, the probe then begins a second 'fine approach' at a slower speed of $2 \mathrm{~nm} / \mathrm{s}$, which allows greater control and data acquisition per $\mathrm{nm}$. This 'fine' approach continues until the dissipative interactions between the probe and fluid cause the amplitude to drop below a 'threshold' value that has been pre-determined by the user. At this point the sample begins a 'fine retraction' at the same $2 \mathrm{~nm} / \mathrm{s}$ rate until the QTF amplitude returns to its original value. After completing this approach retraction sequence, the QTF probe is removed from the microscope and installed in the SEM for characterization. Care is taken to align the QTF probe in the same orientation as the previous characterization, so that the images correspond well. With images of the probe profiles side by side, it is possible to evaluate any eventual plastic deformations which could be attributed to a collision between the gold probe, and solid substrate (meaning the probe has possibly pushed through the adsorbed fluid layer). 


\section{(3.4) Amplitude Dependent Compliance Experiment}

Note: preliminary results of this experiment will be published in Journal of Physics: Conference Series Proceedings of the XVI Meeting of Physics, Lima-Peru 2017 [39], with subsequent results to be published in IEEE Nanotechnology Materials and Devices Conference (NMDC), Portland, 2018 [40].

The experiment outlined above was first performed on February $8^{\text {th }}$ 2018. A pristine sample consisting of a $1 \mathrm{~cm}$ by $1 \mathrm{~cm}$ square of Silicon wafer (with native oxide intact) was prepared by etching in piranha cleaning solution to remove organic contaminants from the surface and render a hydrophilic substrate (as described previously). Before the experiment, a new QTF probe (identified as ADC001) was characterized using the SEM, and a micrograph containing a profile of the probes apex is displayed below in figure 32 a). The experiment began with the 'threshold' value set at an amplitude corresponding to $90 \%$ of the tuning forks original magnitude (as measured far from the surface of the sample). To reiterate, this 'threshold' value is the parameter which determines the point at which the stage stops the 'fine approach', and begin a 'fine retraction'. The experiment was performed at 56\% humidity, with the probe approaching the substrate until the QTF's amplitude decreased to roughly $90 \%$ before proceeding to its retraction as planned. The results of this trial are displayed in figure $32 \mathrm{~b}$ ). In this case, the probe's apex did not show any signs of plastic deformation, meaning that it is unlikely the probe experienced mechanical contact with the solid substrate over the course of the 'approach retraction'

sequence. The experiment was repeated with a new threshold value of $80 \%$ (representing a slightly larger dissipative interaction). Again, the stage approached until QTF's amplitude 
decreased to the set point ( $80 \%$ of the beginning amplitude) and then retracted. The micrograph containing the results of this second trial are displayed in figure $32 \mathrm{c}$ ). In this case deformation of the probe's apex is apparent. It is possible that this deformation could be the result of the probe contacting the solid glass $\left(\mathrm{SiO}_{2}\right)$ substrate. If so, this might be attributed to a non-homogeneous thickness in the adsorbed fluid layer on the surface which could result in the probe contacting a 'dry' region on the wafer. The water layers' formation is a stochastic process that depend on the relative humidity which may render 'islands' of water on a dry substrate [29]. Of course, We may not discard the possibility that this deformation occurred in the process of installing or removing from the microscope head, or loading into the SEM. Ultimately, the accumulation of additional test results will help to reveal the more likely trend. Hence, further experiments were performed, as described below.

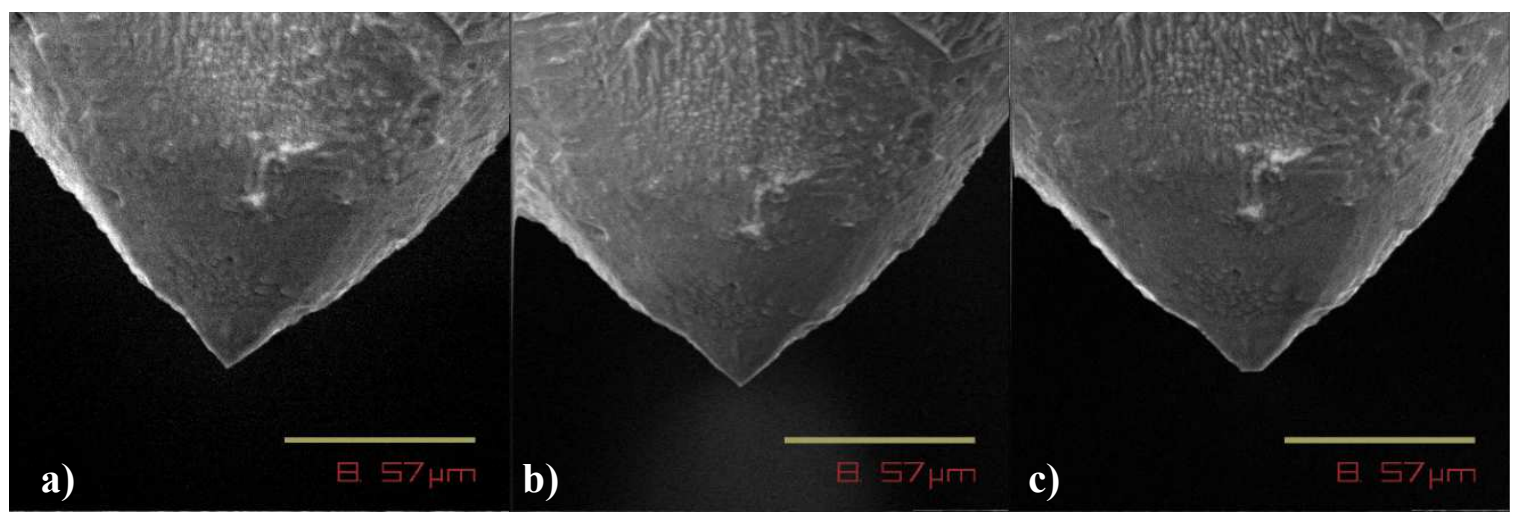

Figure 32 a) SEM imaged captured on 2018-02-08 of QTF probe (ADC001) showing probe morphology before compliance experiment. b) SEM image captured on 20118-02-08 of QTF probe (ADC001) after the amplitude QTF amplitude as decreased from $100 \%$ to $90 \%$, showing no deformation to the probes apex. $\boldsymbol{c}$ ) SEM imaged captured on 2018-02-09 of QTF probe (ADC001) showing probe morphology after the amplitude decreased from $100 \%$ to $80 \%$ and showing plastic deformation to the probes apex. The experiments were performed in frequency modulation modality. 
The experiment was then repeated on 2018-06-13. Again, a pristine piranhacleaned sample was prepared, and a new probe (identified as ADC027) characterized (a micrograph containing a profile of the probes apex is displayed below in figure 33 a) In this trial, the fine approach continued until the QTF's amplitude decreased to $50 \%$ of its original amplitude. At this point, the QTF's resonant frequency had shifted beyond the bandwidth of the PID controller used for frequency modulation causing it to lose 'lock'. Thus, the microscope immediately began the 'fine retraction' process allowing the QTF's amplitude to return to its original value. The probe was characterized as usual by SEM, with micrograph displaying the results in figure $33 \mathrm{~b}$ ).

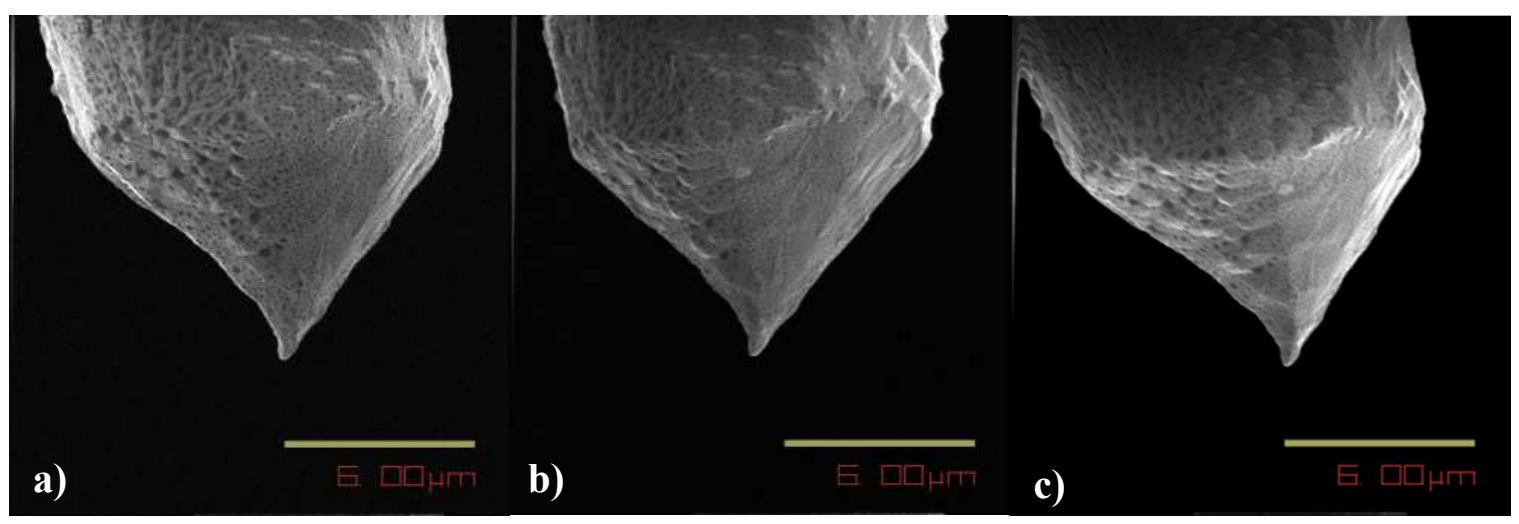

Figure 33 a) SEM imaged captured 2018-06-13 of QTF probe (ADC027) showing probe morphology before compliance experiment. b) SEM imaged captured 2018-06-14 of QTF probe (ADC027) showing probe morphology after the QTF's amplitude decreased from $100 \%$ to roughly $50 \%$; experiment performed under frequency modulation control. c) SEM imaged captured 2018-06-15 of QTF probe (ADC027) showing probe morphology after the QTF's amplitude decreased from $100 \%$ to roughly $25 \%$; experiment performed under constant frequency driving voltage.

In this case, no physical deformation in the probes geometry is visible. The probe even survived the loss of frequency modulation control right after reaching the $50 \%$ amplitude set point. To avoid the loss of PID-controlled frequency modulation that occurred previously, the system then was switched from frequency modulation to constant driving 
frequency mode. In the next trial, the microscope and probe approached until the peak mechanical amplitude (on resonance) decreased to $25 \%$ of the original drive amplitude. This value was calculated in a post processing procedure using the electrical amplitude and phase QTF signals (as described in the previous chapter). The results of this trial are displayed in figure $33 \mathrm{c}$ ). As before, no physical deformation is evident. Clearly there is a discrepancy between the first experiment performed in February, and the second experiment repeated in June. [This results tend to suggest that the observation of probe's apex deformation shown in Fig. $32 \mathrm{c}$ may not be due to the apex-fluid interaction; potentially the apex landed in a region not covered by fluid; or that the water meniscus did not form during the approach as we know that such events are stochastic.]

To investigate the interactions which may have led to the deformation exhibited in figure $32 \mathrm{c}$ ), the interaction damping coefficients and mechanical amplitude were plotted for the experiment relating to figure $32 \mathrm{c}$ ) and figure $33 \mathrm{~b}$ ); these plots are displayed in figures 34 and 35 respectively (both occurrences where the microscope was in frequency modulation). For the trial corresponding the deformation in figure $32 \mathrm{c}$ ), calculation of the probes true mechanical amplitude was conducted in post processing (following the procedure outlined above) and revealed that the amplitude had only decreased to a minimum value of $88 \%$ of its original 18 nanometers (figure 34 left). During this same interaction, the induced damping coefficient registered a peak value of $10 \mathrm{u} S^{-1}$ which is displayed in figure 34 right. 

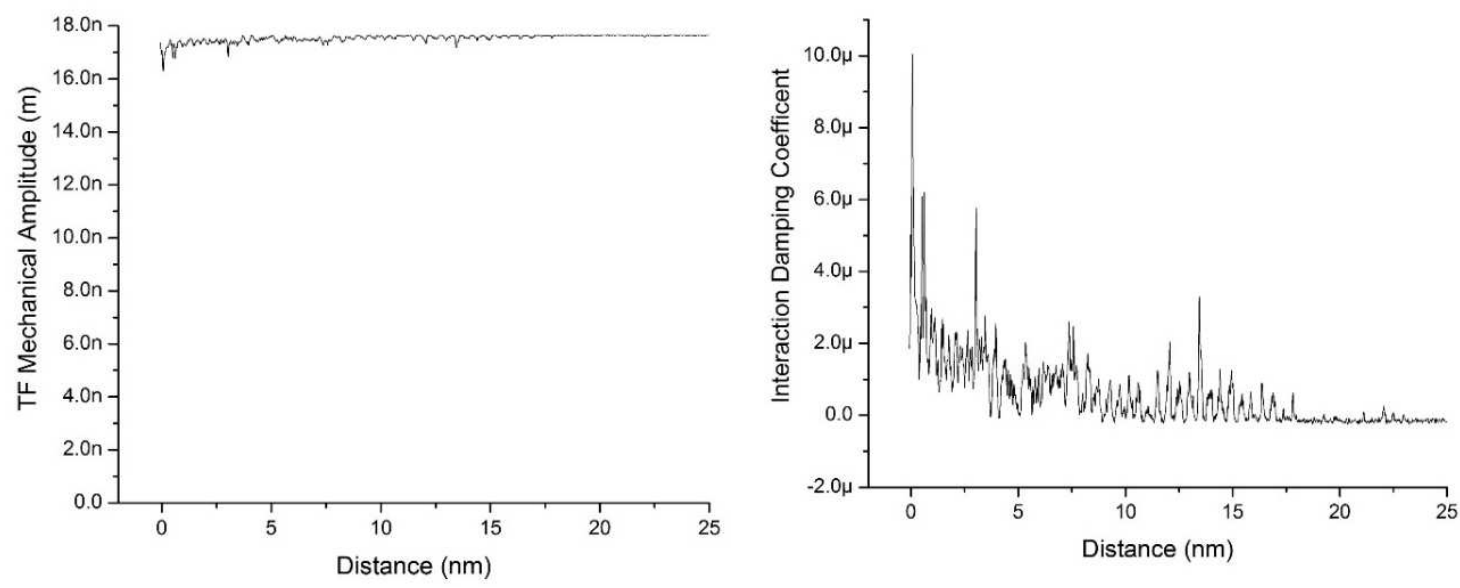

Figure 34 Probe: etched gold (ADC001), Sample: Hydrophilic Si, Humidity: 56\%, original data acquired at $0.4 \mathrm{~ms}$ time constant and $4 \mathrm{~ms}$ data rate. Left) Graph displaying the QTF's mechanical amplitude as a function of distance for the experiment on 2018-02-09 that resulted in the visible deformation of the probes apex seen in figure 30 c). Right) Plot of the interaction damping coefficient as a function of distance for the experiment on 2018-02-09 that resulted in the visible deformation of the probes apex seen in figure 30c). Fluctuations in the plot of interaction damping coefficient is perceived larger than the decrease in mechanical amplitude because the amplitude plotted relative to its absolute zero.
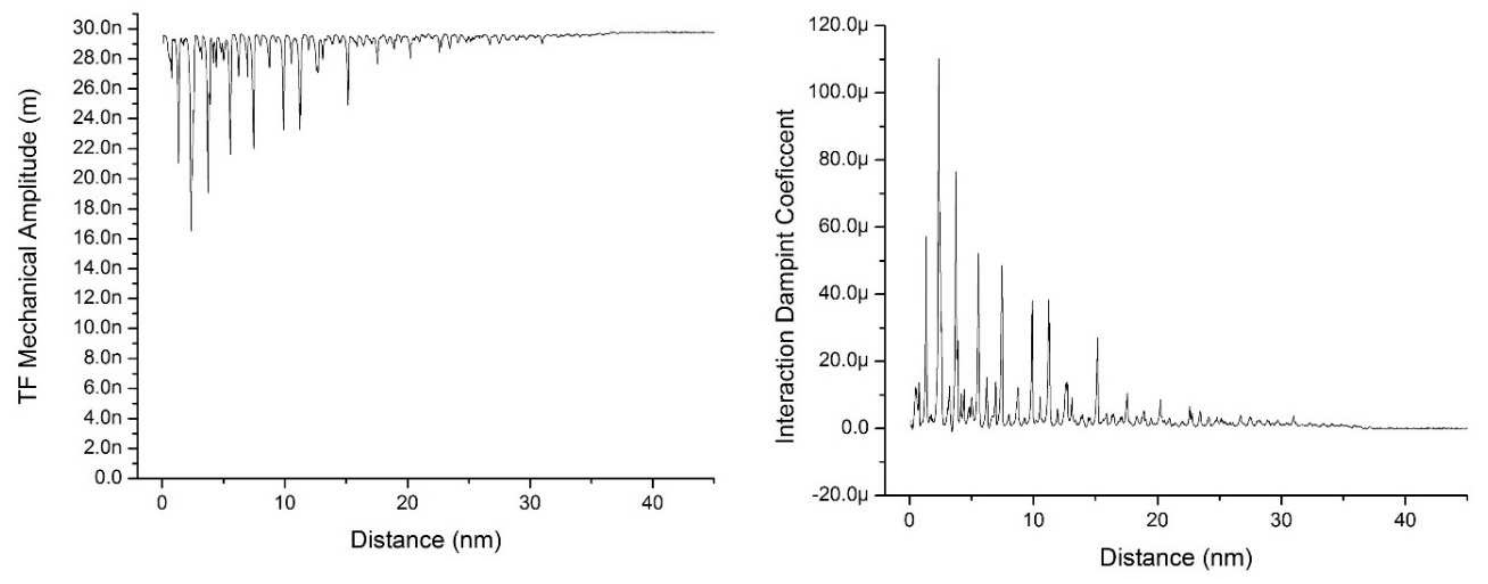

Figure 35 Probe: etched gold (ADC001). Sample: Hydrophilic Si. Humidity: 63.8\%, original data acquired at $0.4 \mathrm{~ms}$ time constant and $4 \mathrm{~ms}$ data rate. Left) Graph displaying the QTF's mechanical amplitude as a function of distance for the experiment on 2018-02-09 that resulted in no visible deformation of the probes apex seen in figure 31b). Right) Plot of the interaction damping coefficient as a function of distance for the experiment on 2018-02-09 that resulted in the no visible deformation of the probes apex seen in figure 31b).

Conversely, Figure 35 indicates that for the trial corresponding to figure $33 \mathrm{~b}$ ), the QTF's mechanical amplitude had reduced to $55 \%$ of its original drive amplitude (figure 35 left). This corresponds to a much larger dissipative interaction than the previous case, which is 
confirmed by analyzing the interaction damping coefficient. In this case, the peak damping coefficient was $110 \mathrm{u} S^{-1}$ (figure 35 right), over ten times the previous experiment where deformation had occurred.

Therefore, the deformation in figure $32 \mathrm{c}$ ), cannot be attributed to a larger magnitude of dissipative damping coefficient, (at least during the monitored approach retraction sequence).

However, it would also be advantageous to analyze the stress on the apex of each probe. Looking at the figures, it can be seen the apex of the probe identified as ADC001 in figure 32 has a radius of approximately $60 \mathrm{~nm}$, while the probe identified as ADC027 has an apex with radius of approximately $100 \mathrm{~nm}$. Meaning the apex of the probe ADDC027 is approximately 2.8 times the surface area of the corresponding probe ADC001 (assuming the interaction area of the probe can modeled as a hemisphere). Because the dissipative force is calculated by multiplying the Interaction Damping Coefficient by the probes velocity, we need to compare the probes average relative velocity. In both cases, the probes are oscillating at roughly the same frequency, meaning the oscillatory period is also roughly the same. However, for the experiment in figure $33 \mathrm{~b}$, the probes amplitude was also roughly twice the oscillating amplitude in experiment from figure $32 \mathrm{c}$, meaning that the average velocity was also approximately twice as large. Therefore, the peak stress on the probe $\mathrm{ADC} 001$ in the experiment shown in figure 34 is proportional to

$$
\text { stress }=\frac{b_{\text {int }} \dot{x}}{A}=\frac{(10 u) v}{2 \pi(60)^{2}}
$$

While the peak stress on probe ADC027 in experiment displayed in figure 35 is 


$$
\text { stress }=\frac{b_{\text {int }} \dot{x}}{A}=\frac{11 *(10 u) 2 v}{2 \pi(100)^{2}}
$$

meaning the stress for the probe displayed in figure $33 \mathrm{~b}$ ) (where no deformation was observed) is still roughly 4 times larger than the case displayed in figure $32 \mathrm{c}$ ) where deformation was observed. Therefore, we can say that the deformation seen in the traces relating to figure 34 was not the result of a yielding interaction stress, as the probe in figure $33 \mathrm{~b}$ experienced an even larger stress without deformation.

In the second experiment, the probe may have interacted in a region on the substrate with robust water layer thickness. In this way, the mechanical damping measured by the probe would be attributed to viscus damping supplied by the adsorbed mesoscopic fluid; as opposed to possibly a dissipative interaction directly between the probe and solid substrate resulting in a plastic deformation displayed in figure $32 \mathrm{c}$ ) (even though the forces magnitude was lower). Of course, it cannot be ruled out that the probe was damaged during loading/ unloading process where the probe was characterized in the SEM.

One possible method to improve reproducibility of these experiments is to have greater control of environmental conditions and sample/substrate cleaning procedures. If the microscope were prepared in an Ultra High Vacuum (UHV) environment, both sample and probe apex could be cleaned with sputter ablation, thereby decreasing the risk of contamination during the current cleaning etch process or while loading samples. Furthermore, a UHV chamber would allow precise control over environmental composition and conditions. 


\section{(3.5) Monitoring Acoustic Emission from Confined Mesoscopic Fluids}

One of the main goals of this thesis, is to investigate the acoustic emission produced by the interactions between a confined mesoscopic fluid and a laterally oscillating, tapered probe. This acoustic signal emission is measured using the SANM acoustic sensor located below the sample.

It has been observed that a tuning fork probe interacting viscoelastically with a sample may produce acoustic emissions after the QTF's oscillation amplitude begins to decrease. To better understand this phenomenon, it would be advantageous to isolate the source of acoustic emissions. One method to ensure that the confined fluid is the source of the acoustic emission (and not perhaps by the probe tapping the substrate) is to verify that the probe has not contacted the substrate prior to phonons being emitted. This can be done by evaluating the probes geometry using SEM (as described in the previous section) before and after the experiment. If the probes apex has contacted the solid substrate beneath the mesoscopic fluid, it should plastically deform (which would be characterized by SEM). In this way, a probe can be characterized before the experiment, installed in the microscope and used to approach a sample until acoustic emission is measured by the SANM sensor, then re characterized after the experiment. If the probe did not show deformation, it would be a good indication that the source of acoustic emission is the confined fluid itself.

This experiment was conducted July $6^{\text {th }} 2018$ using a hydrophobic sample created by etching a $1 \mathrm{~cm} \times 1 \mathrm{~cm}$ section of silicon wafer with dilute Hydro Fluoric acid. A new gold probe identified as ADC029 was also utilized. The microscope approached the sample 
until the SANM signal increased 100\% above the noise level, the results of the experiment are discussed below.

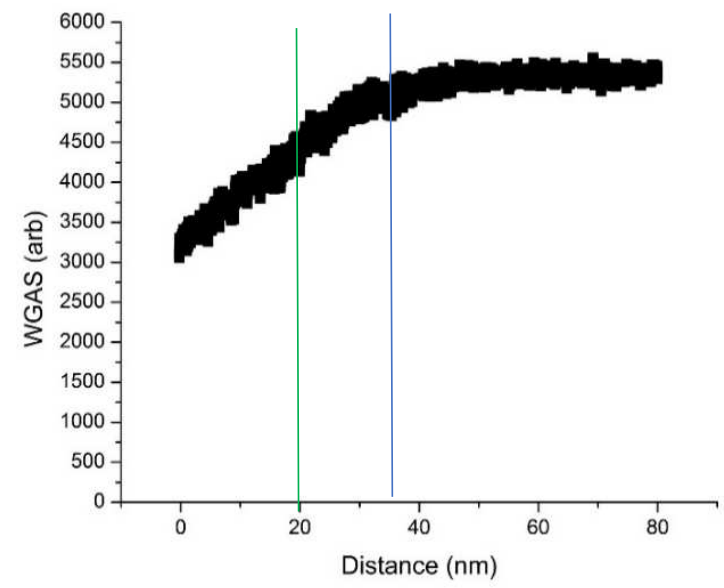

WGAS time constant: $10 \mathrm{~ms}$ Data sample rate: $50 \mathrm{~ms}$

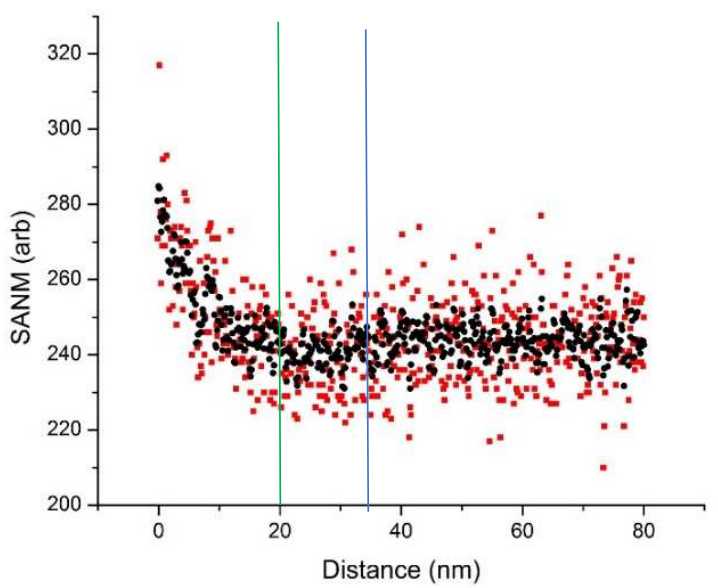

SANM time constant: $10 \mathrm{~ms}$ (RED) SANM sample rate: $50 \mathrm{~ms}$ (BLACK) SANM smoothed (200 pts)

Figure 36 Approach retraction curve between new etched gold probe (ADC029) and hydrophobic HF etched Si sample, humidity: 69.4\%, Left) Plot of WGAS signal as a function of distance for the QTF probe in FM. Right) SANM signal in red displaying the raw data as a function of distance, and black displaying a dataset containing a smoothed SANM signal as a function of distance. The vertical lines help noticing that the acoustic and WGAS signals start to significantly change at a different probe-sample distance.

Figure 36 (left) displays a plot containing the WGAS acoustic signal (which is proportional to the tuning forks mechanical amplitude because the probe is in frequency modulation) which is plotted as a function of distance. Figure 36 (right) is a plot of the acoustic emission as a function of distance measured using the SANM acoustic sensor. This plot contains both the raw SANM acoustic signal as measured from the lock-in amplifier, as well as a digitally filtered dataset that has been smoothed over 200 pts. It is worth noting that the 
SANM plot does not start at zero before the probe starts interacting $(80 \mathrm{~nm}$ on the horizontal scale). This is because the SANM is sensitive enough that it can detect the tuning forks mechanical oscillations through the air. The signal begins to increase at around the $20 \mathrm{~nm}$ scale mark which would signify additional acoustic contribution emitted by the confined fluid. The SANM signal begins to increase when the QTF's mechanical amplitude decreases to roughly $75 \%$ of its original drive amplitude. A comparison of the probes geometry before and after the experiment is included in figure 37.
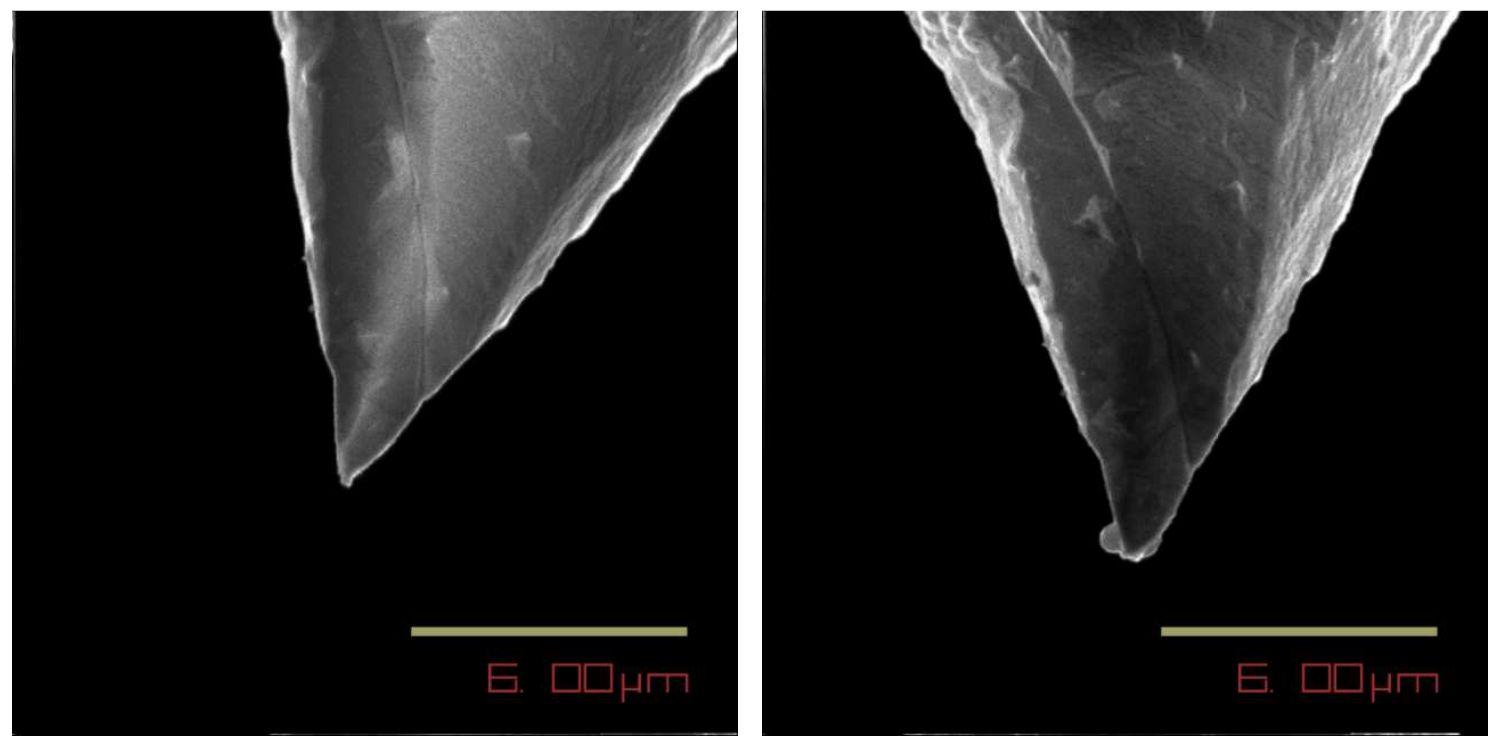

Figure 37 Left) probe identified as ADC029 before the experiment. Right) Probe identified as ADC029 after the experiment. Note possibly slight plastic deformation in the probes apex as well as possible mass transfer.

Possibly a small deformation is visible in the apex of the probe characterized after performing the experiment, (figure 37 right), but there also appears to be some amount of mass transfer. The Hydrofluoric acid (HF) cleaned silicon sample is very hydrophobic, so it is possible that this transferred mass represents accumulated water adhered to the probe, further analysis of chemical composition be necessary for definitive confirmation. 
Another method to ensure that the probe has not contacted the substrate before the acoustic emission takes place would be to approach onto a conductive sample (possibly Highly Ordered Pyrolytic Graphite (HOPG)) while simultaneously monitor the tunneling current and SANM response. This method however presents its own limitations as some tunneling will take place through the water layer, and it is not always a straight forward process to interpret when contact between the probe and substrate occurs (depending on process such as band gap, and tunneling potential) [41].

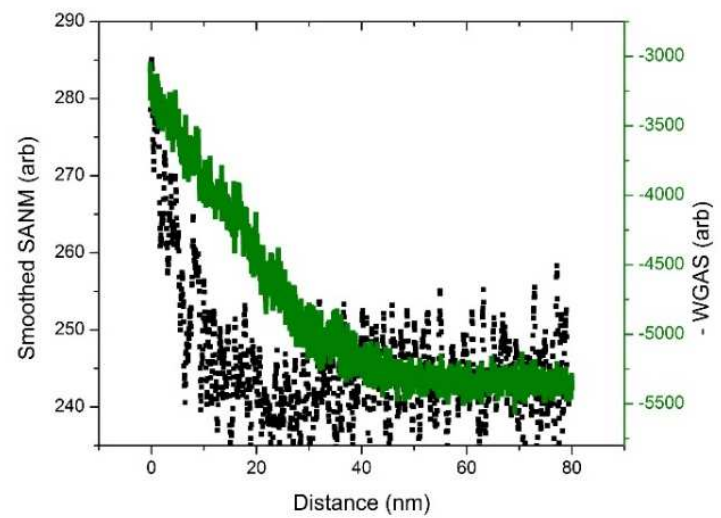

SANM time constant: $10 \mathrm{~ms}$

WGAS time constant: $10 \mathrm{~ms}$

(BLACK) SANM sample rate: $50 \mathrm{~ms}$ (BLACK) smoothed SANM (200 pts) (GREEN) WGAS sample rate:50 ms

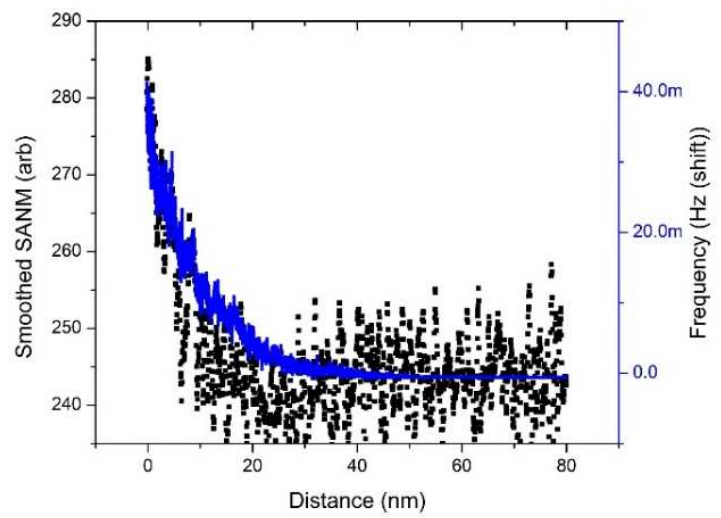

SANM time constant: $10 \mathrm{~ms}$ Frequency Shift time constant: 400 us (BLACK) SANM sample rate: $50 \mathrm{~ms}$ (BLACK) smoothed SANM (200 pts) (Blue) Frequency Shift sample rate: $4 \mathrm{~ms}$

Figure 38 Approach retraction curve between new etched gold probe (ADC029) and hydrophobic HF etched Si sample, Humidity: $69.4 \%$. Left) dataset containing a smoothed SANM signal against the inverse of the WGAS signal, showing poor correlation between the two signals. The source of acoustic emission is not of damping nature then. Right) dataset containing a smoothed SANM signal against the resonant frequency shift showing better correlation. This is in agreement with the consideration that the acoustic emission (WGAS signal) is an elastic energy-dissipation channel (reflected in the frequency shift) of the probe-fluidsubstrate interaction.

Last, to understand the origins of this acoustic emission, it would be beneficial to examine the SANM acoustic response in relationship the other signals. When the filtered 
SANM signal is plotted against the WGAS (which has been multiplied by -1 to aid in graphical comparison) the two plots are not well correlated (figure 38 left). The WGAS signal (representing the QTF's mechanical amplitude) begins to shift about $20 \mathrm{~nm}$ before the SANM. This would suggest that the acoustic emission is not likely linked to a dissipative channel for energy. However, when the SANM signal is plotted against the shift in resonant frequency, there is a better correlation between the two signals. In both cases, the signals begin to shift at the $20 \mathrm{~nm}$ mark and display non-monotonic behavior figure 38 right.

\section{(3.6) Hydrophobic vs Hydrophilic Sample Response}

Having used the microscope to analyze a variety of samples, it is apparent that the substrate's hydrophobicity has a measureable effect on the probe-sample interaction, as well as the resulting approach retraction curve. Hydrophobic samples such as HF etched Silicon, appear to produce approach-retraction curves where the tuning fork amplitude smoothly decreases as the separation distance between sample and probe decrease, and interaction forces increase. Hydrophilic sample may generate approach retraction curves with a much more drastic change in probe amplitude. To explore this phenomenon, a comparison will be made between curves generated on a hydrophobic substrate against those made on a hydrophilic substrate. To isolate the role of sample hydrophobicity, as many variables a possible were held constant. In both cases, trials were selected where the QTF's amplitude decreased by approximately half, both probes exhibit similar sized apex geometry, and both trials were chosen after the probe had previously interacted with the 
sample several times (i.e. not first interactions). Perhaps most importantly though, both trials were conducted using the frequency modulation modality. With these parameters held constant, the differences in approach-retraction curves of the two cases may be attributed to the variations in sample hydrophobicity

First, a hydrophobic Si sample was investigated utilizing curves collected on 201807-06, where an etched gold probe (identified as ADC029) was utilized (see figure 39 left). Approach curve labeled 1a12 was selected for analysis and can be viewed in figure 40 (upper left for approach portion, and lower left for retraction). A smooth transition in the QTF's amplitude as a function of distance is visible.

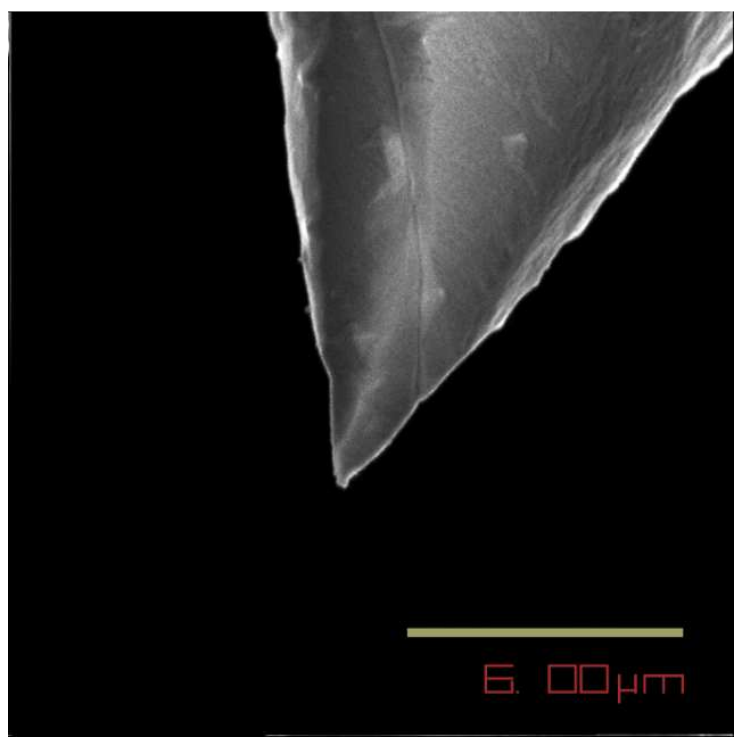

Probe used for curve: $1 \mathrm{a} 12$

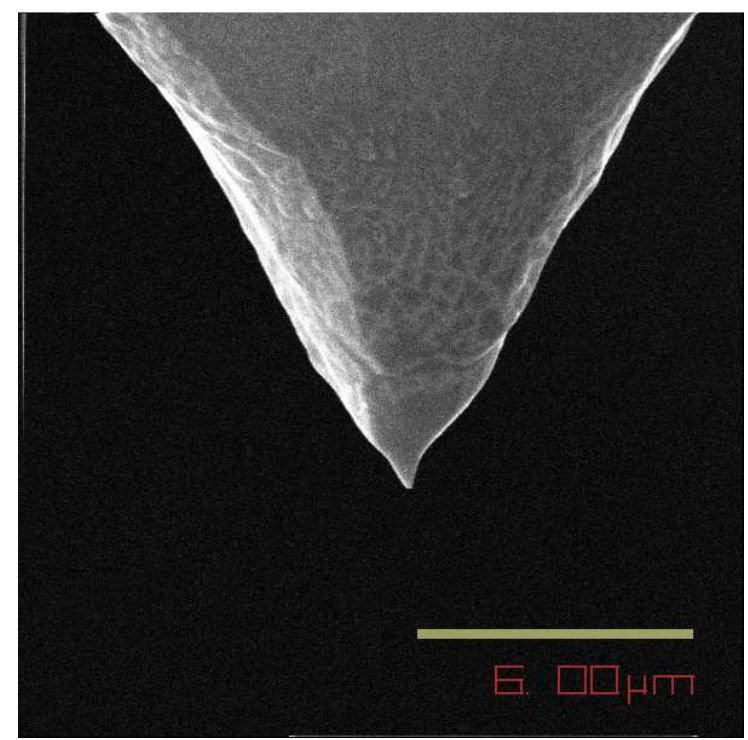

Probe used for curve: $5 \mathrm{a} 1$

Figure 39 Left) SEM image of probe identified as ADC029, used on hydrophobic sample (HF etched Si) to generate the plots displayed in figure 38 (left above, and left below). Right) SEM image of probe identified as $\mathrm{ADC} 025$, used on hydrophilic sample (Piranha cleaned Si) to generate plots displayed in figure 38 (right above, and right below). 
A different story is evident when examining approach retraction curves collected on 201807-02 using a hydrophilic Si sample. Again, an etched gold probe (identified as ADC025) (see figure 39 right) was used to approach a sample of piranha cleaned Si. In this case, as the distance between the probe and sample decreases, there is an abrupt decrease in the probes mechanical amplitude, as displayed in the WGAS plot of figure 40 (top right for approach, and bottom right for retraction). In this instance, the amplitude decrease appears predominantly in the retraction portion of the curve, which is likely due to the relatively large time constant causing a delay in data acquisition. This difference in curve geometry as a function of substrate hydrophobicity can be explained by exploring the details of the probe-mesoscopic fluid interaction. From the experimental observations of Major et al, it is clear, the samples hydrophobicity effects how the adsorbed water layers one the probe, and substrate, interact. In my hydrophobic sample, the probes amplitude exhibits a smooth decrease in amplitude. This is the same results reported by Major et al for hydrophobic samples where film-film contact occurs likely without a predominant formation of a meniscus water bridge [34]. The immediate amplitude change for my hydrophilic case, also corresponds to an immediate change in viscus damping. Again, this finding is corroborated with results reported by Major et al, where a sharp transition in friction force was recorded using hydrophilic sample and substrate [34]. 

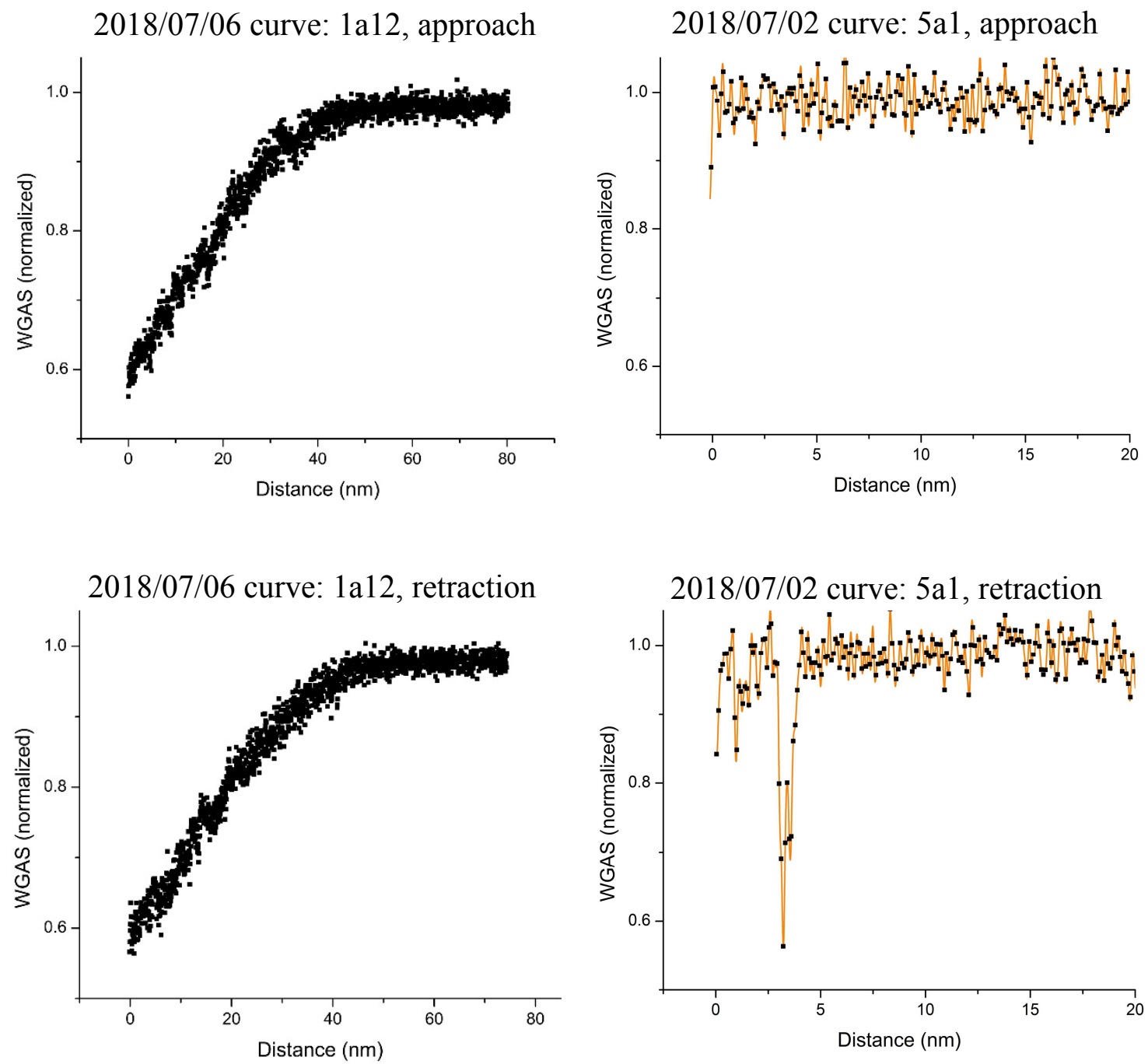

Figure 40 Upper left) Plot of WGAS approach curve using probe identified as ADC029 on hydrophobic sample (HF etched Si). Upper right) Plot of WGAS retraction curve using probe identified as ADC029 on hydrophobic sample (HF etched Si). Lower left) Plot of WGAS approach curve using probe identified as ADC025 on hydrophilic sample (piranha cleaned Si). Lower right) Plot of WGAS retraction curve using probe identified as ADC025 on hydrophilic sample (piranha cleaned $\mathrm{Si}$ ). In all cases lock-in time constant: $10 \mathrm{~ms}$, data rate:50 ms.

Furthermore, it appears that the rate at which the viscus damping force is experienced by the probe, is dependent on relative humidity, Major et al showed that as the relative humidity increased from $10 \%$ to $45 \%$, the transition in friction force became more immediate. The hydrophobic results presented here on 2018/07/02 exhibiting an immediate 
onset of viscus damping (as evident in the probe amplitude decrease in frequency modulation) were taken at $62 \%$ humidity again corroborating previous findings, These results appear to confirm meniscus formation in hydrophilic samples.

\section{(3.7) Evolution of Approach Curves Under Increasing Probe Sample Interactions}

Often when analyzing approach-retraction curves in post processing, curve trends will appear to evolve as the number of interactions increase. Many times, the first few interactions appear more chaotic, or exhibit a larger noise level. However, as the number of interactions, and approach-retraction curves increase, the curves become more reproducible, and exhibit a modified shape. To explore this, a series of approach-retraction curves were performed on June $20^{\text {th }} 2018$, utilizing a HOPG substrate, and tapered gold probe identified as (ADC027). HOPG was selected because unlike the previous samples studied it is nether extremely hydrophobic or extremely hydrophilic. Figure 41 displays WGAS traces of first probe-sample interaction of the sequence on the left, and $12^{\text {th }}$ consecutive interaction on the right. At the beginning, (first approach) the WGAS trace (proportional to the QTF displacement) exhibits a smooth decrease in amplitude for roughly the first $60 \mathrm{~nm}$. After this point though, there appears to be a 'bump' at $20 \mathrm{~nm}$, where the tuning forks displacement increases slightly (indicative of a decrease in viscus integration forces). This bump correlates well with results published by Monte Kozell in his thesis [42], using a separate AFM microscope in this lab. Major et al also reported a similar 'bump' in hydrophilic samples and attributed this to the fluid later being' squeezed out'. This trace is interesting because it illustrates the initial wetting. This hybrid shape 
may be the result of an unstable meniscus formation caused by using a gold probe which is not highly hydrophilic.

In comparison, the $12^{\text {th }}$ trace (figure 41 right) displays a more immediate decrease in amplitude around the $65 \mathrm{~nm}$ mark followed by a monotonic decrease in amplitude until $35 \mathrm{~nm}$, at which point a second monotonic decrease in amplitude (at slower rate) is followed. This dual liner behavior has been reported previously in hydrophobic samples. Again, the trace seems to exhibit hybrid behavior. More analysis is required, but I postulate that evolution in the curve profile is due to a mass transfer (of the adsorbed fluid) from the sample to the probe. In the first trace, it appears that a mesoscopic water bridge doesn't abruptly form, but eventually as the wetting process occurs, a meniscus does form, which causes the abrupt transition in QTF amplitude. However, the later traces do not appear to exhibit the 'squeeze out' phenomena', possibly due to capillary action drawing an increase in fluid to the probe substrate region.
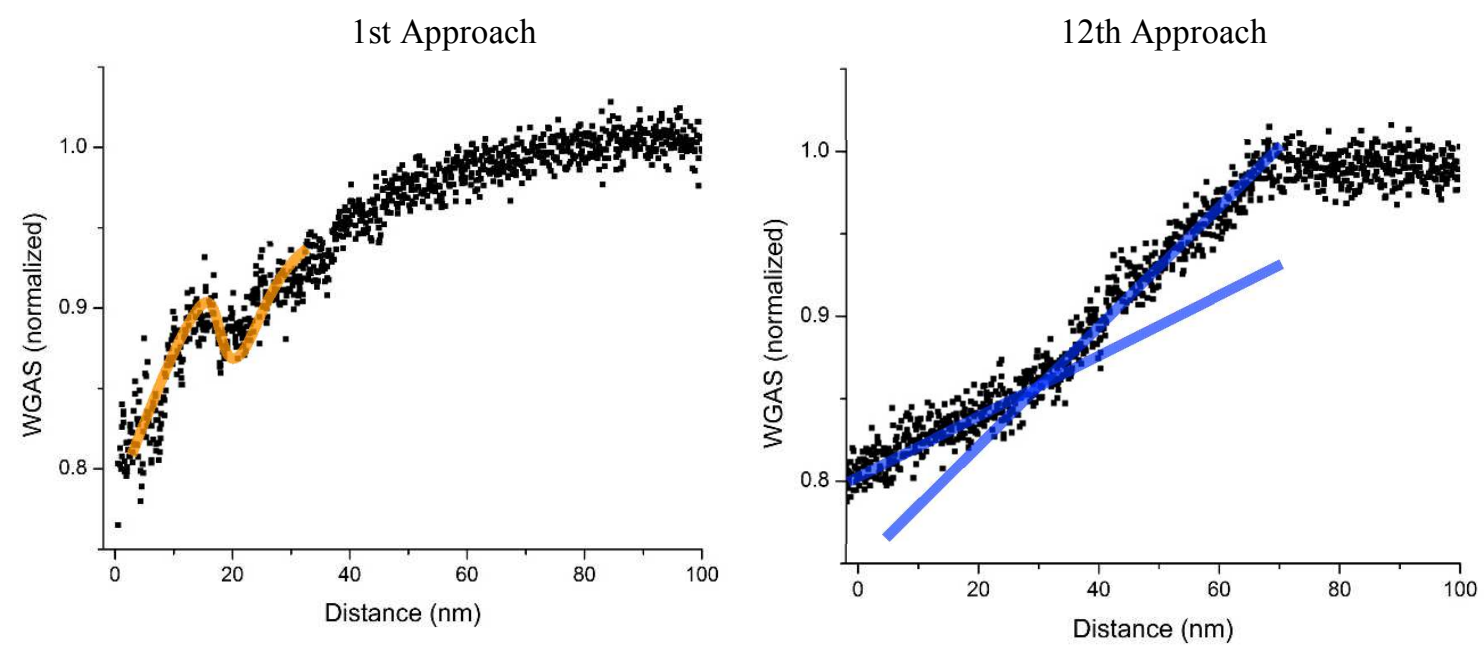

Figure 41 Approach retraction curves using an etched gold probe and HOPG sample at $63 \%$ humidity. WGAS time constant: $10 \mathrm{~ms}$, Data rate: $50 \mathrm{~ms}$. Left) a the $1 \mathrm{st}$ interaction Right) $12^{\text {th }}$ interaction. 


\section{(3.8) Next Steps}

A brief section in the previous chapter disused the construction of AFM based sheer force probes. This avenue that will be pursued in future iteration of this microscope. As mentioned earlier, these probes exhibit tip geometries that are incredibly consistent. This is consistency is important as it allows simple comparison of sheer force measurements between multiple trials, without necessarily evaluating relative sheer interactions as done in section (3.4) when probes of slightly different tip geometry were analyzed. Furthermore, the production AFM probes composed of Silicon are much more robust against compliance and deformation. The poor-quality SEM micrographs displayed below in figure 42 were captured in September of 2016 after the technique was first developed.
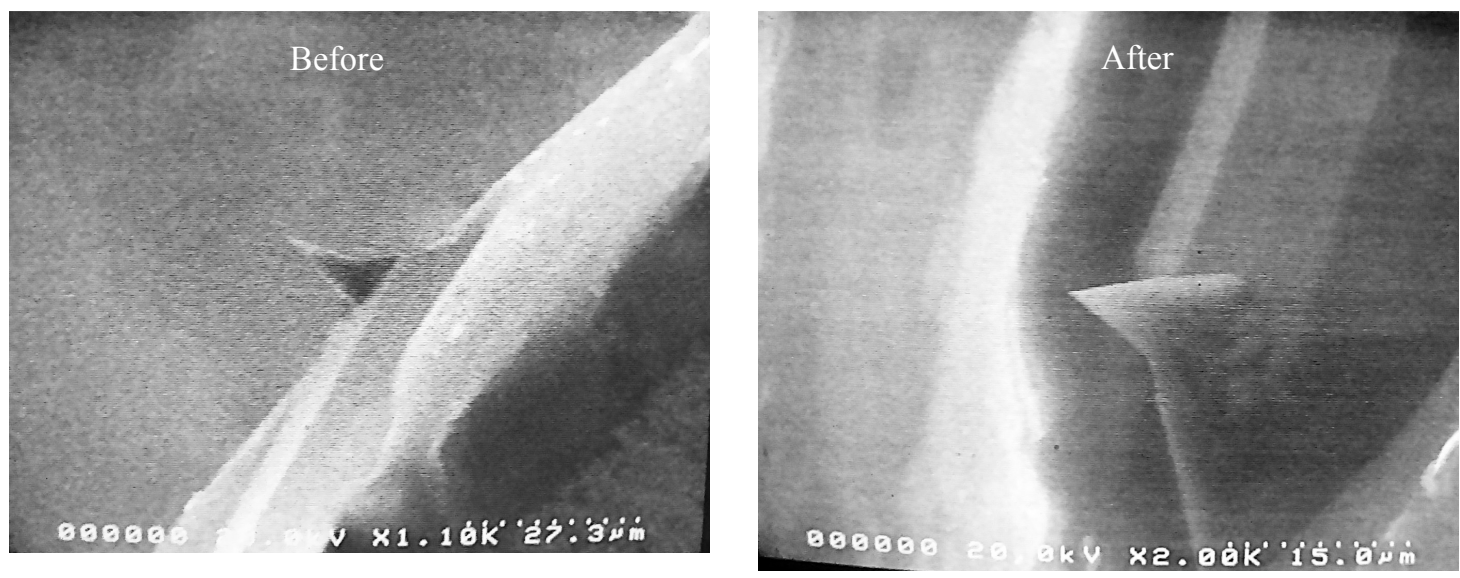

Figure 42 SEM micrograph of an early AFM probe. Left) the initial characterization after probe fabrication, Right) subsequent characterization after interacting with sample.

Figure 42 left displays an AFM probe being characterized immediately after being produced. The probe was then used to approach a Silicon sample with single layer of hydrophilic Poly(2-ethyl-2-oxazoline) (PEOX) deposited on top. The probe approached 
the sample until the oscillation amplitude as measured by the WGAS sensor (in frequency modulation) decreased to roughly $45 \%$ of its initial value. Figure 42 right displays the same AFM probe being characterized after this interaction, revealing no measureable deformation even when approaching the hard silicon substrate. This result further validates the finding of section 3.4 .

It is difficult to directly compare the approach retraction curves using an AFM probe, with those utilizing etched gold probes. The AFM probes are sharper, less compliant, and likely more hydrophobic than the etched gold probes. However, even with these variables, it is possible to note a few interesting observations. The approach retraction curves corresponding to the AFM probe interacting with the PEOX sample are displayed below in figure 43. The probes amplitude (as viewed in the WGAS trace on figure 43 a) begins to decrease linearly from $100 \%$ at $70 \mathrm{~nm}$ mark (noted with the vertical blue line labeled 'A'), to $75 \%$ at $30 \mathrm{~nm}$ 's (noted with the vertical blue line labeled 'B'). This decreased amplitude represents a viscus interaction. During this same interval, the systems resonant frequency linearly increased to several $\mathrm{mHz}$ (corresponding to an elastic interaction, see figure $43 \mathrm{~b}$ ). Noting the finding of Major et al, position A likely represents the point of fluid nucleation [34]. There does not appear to be a clear capillary condensation point, however this is not suppressing, as the silicon AFM probe is relatively hydrophobic, (Major et al similarly do not report capillary condensation using a combination hydrophobic/hydrophilic tip sample combination).

Around point 'B', the WGAS trace increases slightly (decreasing viscus force) creating the bump, a characteristic indication found from hydrophilic samples; Again, this 
bump can be attributed to the meniscus being squeezed from the Nano gap between the probe and sample [34]. As the frequency increases at point B, an acoustic emission is observed (see figure $43 \mathrm{c}$ ) just as it was in figure 38. This SANM signal increasing to a maximum around $8 \mathrm{~nm}$ (point $\mathrm{C}$ ). point ' $\mathrm{C}$ ' is noteworthy as each of the plots display distinctive properties. Here (C), the WGAS trace has a slight kink, the noise level of the resonant frequency shit increases, and the SANM trace begins to decrease. Because these observables all occur at the same point, it may be indicating an interface, or a change in the mesoscopic fluids material properties. If the SANM's acoustic emission is in fact related to a channel of elastic interaction with the mesoscopic fluid, it is interesting that the probes resonant frequency did not simultaneously decrease at this point.

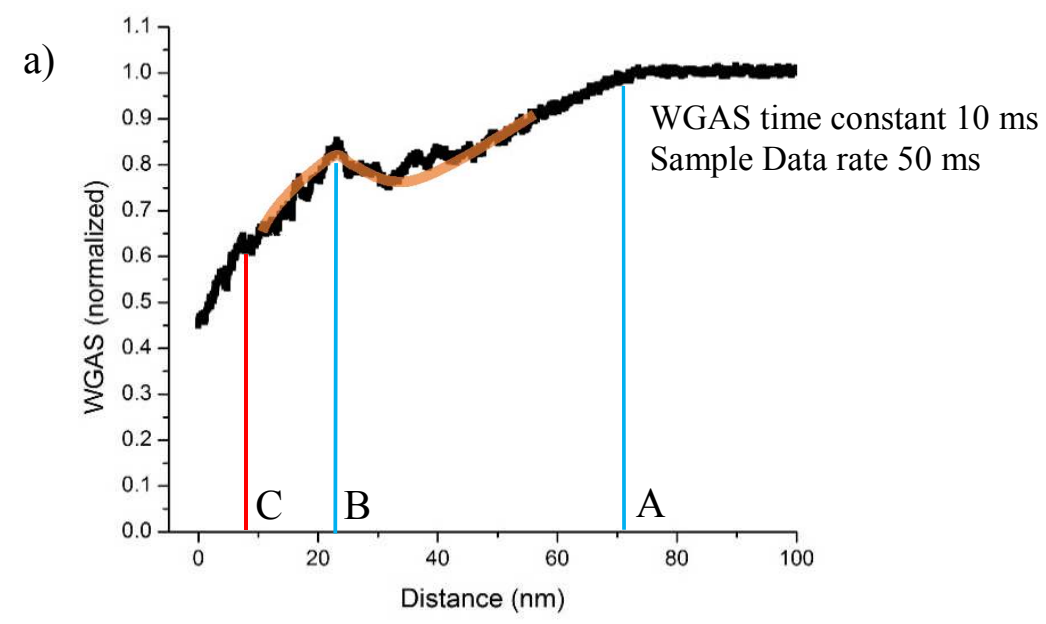


b)

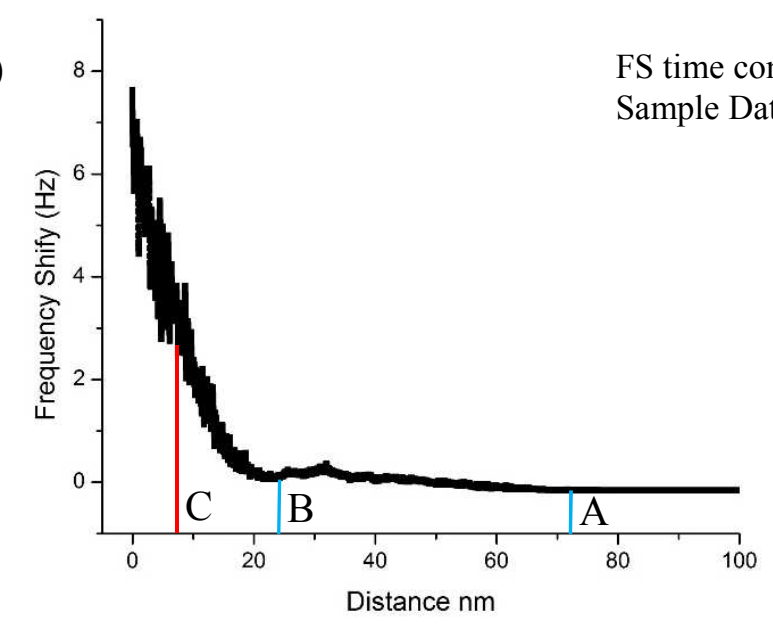

c)

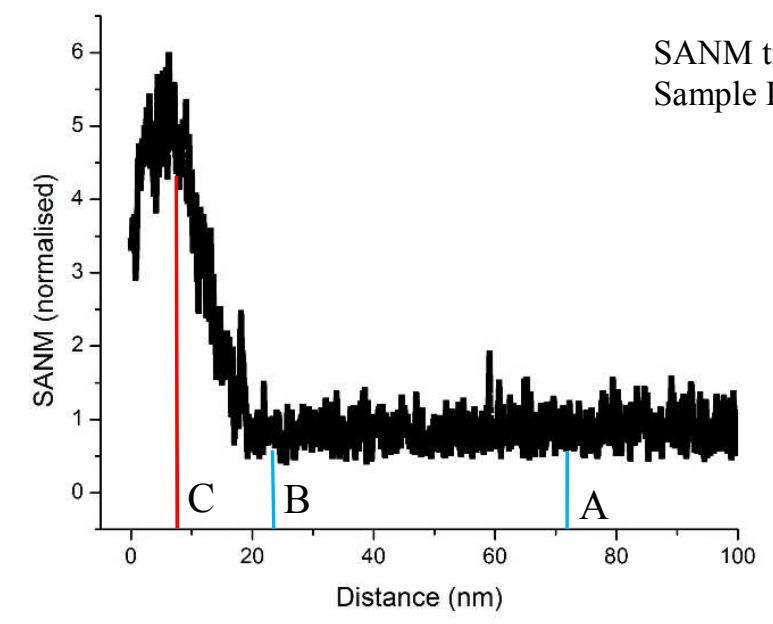

Figure 43 Approach retraction curve using AFM probe and Poly(2-ethyl-2-oxazoline) sample, Humidity is unknown. a) A plot of the WGAS sensor as a function of distance. This trace from the approach retraction curve using the AFM tip. The magnitude of the trace has been normalized such that a magnitude of 1 refers to the probes amplitude away from the surface. B) A plot of the AFM probes resonant frequency shift as a function of distance. C) A plot of the SANM sensor as a function of distance. Here the sensors magnitude has been normalized such that 1 refers to the sensors acoustic reading while the probe and sample are not interacting.

Perhaps $\mathrm{C}$ represents a point near the substrate fluid interface where the probes apex is interacting with monolayers, while the sides of the probe interact with the bulk fluid. Further investigation will a larger dataset using AFM probes will be required. The acoustic data presented in figure $43 \mathrm{c}$ ) is important, because we have not before observed the 
acoustic emission peak, then decrease in frequency modulation. The apexes of our etched gold probes typically have a larger radius than the production AFM probes This means that less sheer stress may be applied to the probe under similar conditions, allowing the probe to interact deeper into the meniscus before losing lock with the PLL which drives the frequency modulation (as the systems resonant frequency shift is typically exponentially related to the probe-substrate separation).

\section{(3.9) Conclusions}

In this thesis, the recently developed Shear-Force Acoustic Near-Field Microscope was improved, discussed, and used to investigate the confinement of an adsorbed mesoscopic fluid layer between two trapping boundaries (consisting of the stationary substrate, and the apex of a tapered, laterally oscillating probe). It was shown that under sufficiently high humidity, electromechanically etched tapered gold probes are sufficiently robust enough to withstand viscous interactions with a confined mesoscopic fluid until the amplitude of oscillation decreases to $25 \%$ of its original amplitude without displaying visible plastic deformation (within the precision of the measurement tools available). Acoustic emission from a confined mesoscopic fluid was also investigated using the SANM acoustic transducer. It was found that the acoustic emissions exhibit a behavior closer to (if not coincident with) the QTF's resonant frequency shift behavior as the probe approaches the substrate (at least in the first $50 \mathrm{~nm}$ of interaction). In both cases, the traces begin to shift at the same probe-sample point, and follow a similar monotonic shift (not correlated to the QTF amplitude), which suggests the acoustic emission is related to an 
elastic interaction. Next, hydrophobic interactions between the probe and sample were discussed. It was found that when testing hydrophobic samples (such as HF etched $\mathrm{Si}$ ), the approach-retraction traces exhibit a smooth and continuous decrease in probe oscillation amplitude (with increasing interaction distance) indicative of a weaker coupling between the adsorbed fluid and substrate. Conversely, highly Hydrophilic samples (like piranha cleaned $\mathrm{SiO}_{2}$ ) exhibit a much more drastic decrease in probes oscillation amplitude with the onset of interaction. In the latter case, this interaction could be indicative of a meniscus 'water bridge' forming between the probe and sample thereby viscously damping oscillations. Furthermore, the wetting process was investigated by observing how probesample sheer interactions evolve as the number of approach-retraction curves increase. Here the first interactions between an etched gold probe and HOPG sample exhibit hydrophilic style interaction curves, while later interaction curves exhibit more hydrophobic interactions. This may be caused by mass transfer of the adsorbed fluid layer to the probe. Finally, preliminary data collected using a new AFM based probe and hydrophilic sample was presented. This data showed an interesting link between the acoustic emissions and the elastic interaction in frequency modulation, which was made possible due to the small radius, and rigidity afforded by the Silicon probe. While a trend of reproducibility is reflected from the multiple approach-retraction tests performed herein, reproducibility of the results under well controlled of humidity and very pristine conditions, (ideally starting from very high vacuum $\left(10^{-9} \mathrm{Torr}\right)$ conditions followed by gradual changes towards ambient conditions) would place the study of mesoscopic fluids under stronger grounds. 


\section{Works Cited}

[1] P. H. Anjos, E. O. Dias, L.' . Dias and J.' . A. Miranda, "Adhesion force in fluids: Effects of fingering, wetting, and viscous normal stresses," Physical Review E, vol. 91, no. 1, pp. 013003--7, 7 January 2015.

[2] T. M. Squires, "Microfluidics: Fluid physics at the nanoliter scale," Reviews of Modern Physics, vol. 77, pp. 977-1026, July 2005.

[3] D. Schäffel, K. Koynov, D. Vollmer, H.-J. Butt and C. Schönecker, "Local Flow Field and Slip Length of Superhydrophobic Surfaces," Physical Review Letters, vol. 116, pp. 134501-5, 1 April 2016.

[4] I. Walker, T. S. Li and c. Gilbert, "Single Polymer Studies of Hydrophobic Hydration," Accounts of Chemical Research, vol. 45, no. 11, pp. 2011-2021, 8 may 2012.

[5] M. D. Ma, L. Shen, J. Sheridan, J. Zhe Liu, C. Chen and Q. Zheng, "Friction of water slipping in carbon nanotubes," Physical Review E, vol. 83, pp. 036316-1, 2011.

[6] R. Wiesendanger, Scanning Probe Microscopy and Spectroscopy, New York, New York: Cambridge University Press, 1994, pp. 81-273.

[7] D. Tabor and R. H. S. Tabor, "The Direct Measurement of Normal and Retarded van der Waals Forces," Proceedings of the Royal Society of London, vol. 312, no. 1511, pp. 435-450, 30 September 1969.

[8] E. Teague, "Three-dimensional stylus profilometry," Wear, vol. 83, no. 1, pp. 1-12, 1982.

[9] G. Binnig, H. Rohrer, C. Gerber and E. Weibel, "Tunneling Through a Controllable vacuum gap," Applied Physics Letters, vol. 40, no. 2, pp. 178-180, 15 January 1982.

[10] G. Binnig and H. Rohrer, "Scanning Tunneling Microscopy," Surface Science, vol. 126, pp. 236-244, 1983.

[11] G. Binning and C. F. Quate, "Attomic Force Microscope," Physical Review Letters, vol. 56, no. 9, pp. 930-933, 3 march 1986.

[12] G. Meyer and N. m. Amer, "Novel optical approach to atomic force microscopy," Applied Physics Letters, vol. 53, no. 12, pp. 1045-1047, 19 September 1988.

[13] C. M. Mate, G. M. McCelland, R. Erlandsson and S. Chiang, "Atomic-Scale Friction of a Tungsten Tip on a Graphite Surface," Physical Review Letters, vol. 59, no. 17, pp. 1942-1945, 26 October 1987.

[14] E. Betzig, P. L. Finn and J. S. Weiner, "Combined shear force and near-field scanning optical microscopy," Applied Physics Letters, vol. 60, no. 20, pp. 24842486, 18 May 1992.

[15] K. Karrai and R. D. Grober, "Piezoelectric tip-sample distance control for near field optical microscopes," Applied Physics Letters, vol. 66, no. 14, pp. 1842-1844, 3 April 1995. 
[16] P. Gunther, U. C. Hischer and K. Dransfeld, "Scanning Near-Field Acoustic Microscopy," Applied Physics B, vol. 48, no. 89, pp. 89-92, 24 October 1989.

[17] K. Karrai and T. Ingo, "Interfacial shear force microscopy," Physical Review B, vol. 62, no. 19, pp. 13174-13181, 8 May 2000.

[18] A. H. La Rosa, X. Cui, J. McCollum, N. Li and R. Nordstrom, "The ultrasonic/shear-force microscope: integrating ultrasonic sensing into a near-field scanning optical microscope," Rev. Sci. Instrum, vol. 76, pp. 093707-1-6, September 2005.

[19] H. A. La Rosa, N. Li , R. Fernandez, X. Wang, R. Nordstrom and S. K. Padigi, "Whispering-gallery acoustic sensing: characterization of mesoscopic films and scanning probe microscopy applications," Rev. Sci. Instrum, vol. 82, pp. 093704 (18), September 2011.

[20] J. M. Friedt and E. Carry, "Introduction to the quartz tuning fork," American Journal of Physics, vol. 75, no. 5, p. 415, 2007.

[21] S. K. Mondal, A. Mitra, N. Singh, S. N. Sarkar and P. Kapur, "Optical fiber nanoprobe preparation for near- field optical microscopy by chemical etching under surface tension and capillary action," Optics Express, vol. 17, no. 22, pp. 1947019475, 2009.

[22] S. Narasiwodeyar, M. Dwyer, M. Liu, W. K. Park and L. H. Greene, "Automated fabrication technique of gold tips for use in point-contact spectroscopy," Review of Scientific Instruments, vol. 86, no. 3, 2015.

[23] Nanonis GmbH, "Piezoelectric Quartz Tuning Forks for Scanning Probe Microscopy," 2005, Whitepaper.

[24] R. Oria, J. Otero, L. González, L. Botaya, M. Carmona and M. Puig-Vidal, "Finite Element Analysis of Electrically Excited Quartz Tuning Fork Devices," Sensors, vol. 13, pp. 7156-7169, 2013.

[25] T. Le, H. Trans, R. Fernandez, N. Laal, A. H. La Rosa, C. J. S. Salinas, R. Bringas, J. Quispe and F. Segundo, "Finite Element Method Analysis of Whispering Gallery Acoustic Sensing," in Proceedings XVI Meeting of Physics, Lima-Peru, 2017.

[26] A. H. La Rosa, X. Cui, J. McCollum, N. Li and R. Nordstrom, "The Ultrasonic/Shear-Force Microscope: Integrating Ultrasonic Sensing into a NearField Scanning Optical Microscope," Review of Scientific Insturments, vol. 76, p. 093707, 2005.

[27] Rodolfo Fernandez Rodriguez, "Confined Mesoscopic Fluid-like Films Analyzed with Frequency Modulation and Acoustic Detection," 2014, Thesis.

[28] J. Karki, "Active Low-Pass Filter Design," 2002, Whitepaper.

[29] J. Freund, J. Hailbritter and j. Hörber, "How Dry Are Dried Samples? Water Adsorption Measured by STM," Microscopy Research and Technique 44:327-338 (1999), vol. 44, 1999. 
[30] L. Greenspan, "Humidity Fixed Points of Binary Saturated Aqueous Solutions," Journal of Research of the National Bureau of Standards - A. Physics and Chemistry, vol. 81, no. 1, pp. 89-96, 1977.

[31] M. Luna, J. Colchero and A. M. Baro', "Intermittent contact scanning force microscopy: The role of the liquid necks," Applied Physics Letters, vol. 72, no. 26, pp. 3461-3463, 29th June 1998.

[32] J. Israelachvili, Intermolecular \& Surface Forces, 2nd, Ed., Santa Barbra , CA: Acedemic Press, 2007.

[33] R. Digilov, "Kelvin Equation for Meniscuses of Nanosize Dimensions," Langmuir, vol. 16, no. 3, pp. 1424-1427, 2000.

[34] R. C. Major, J. E. Houston, M. j. McGrath, J. I. Siepmann and X. Y. Zhu, "Viscous Water Meniscus under Nanoconfinement," Physical Review Letters, vol. 96, pp. 177803-4, 6 May 2006.

[35] Portland State University, "Hydrofluoric Acid Use at PSU," [Online]. Available: https://www.pdx.edu/environmental-health-safety/hydrofluoric-acid-use-at-psu. [Accessed 30 September 2018].

[36] X. Zhang and E. Garfunkel, "Stability of HF-etched Si(100) surfaces in oxygen ambient," Applied Physics Letters, vol. 79, no. 4, pp. 4051-4053, 10 December 2001.

[37] S. Kaya, P. Rajan, H. Dasari, D. C. Ingram, W. Jadwisienczak and F. Rahmant, "A Systematic Study of Plasma Activation of Silicon Surfaces for Self Assembly," Applied Chemical Society, vol. 7, p. 25024-25031, 28 october 2015.

[38] R. Fernandez, X. Wang and A. H. La Rosa, "Acousto Characterization of Fluid-like Mesoscopic Films Under Shear," in Proceedings of the Nanotechnology (IEEENANO) 11th IEEE International Conference, 2011.

[39] T. A. Brockman, R. Fernandez, J. Bai, M. Kozell and A. H. La Rosa, "Probe Damage Evaluation in Frequency-Modulation Shear-force Acoustic Near-field Microscopy," in Journal of Physics: Conference Series Proceedings of the XVI Meeting of Physics, Lima-Peru, 2017.

[40] T. Brockman, A. La Rosa, J. Bai and R. Fernandez, "Assessing Probe Damage in Constant Frequency and Frequency-Modulation Shear-force Acoustic Near-field Microscopy," in IEEE Nanotechnology Materials and Devices Conference (NMDC), Portland, 2018.

[41] J. R. Hahn, Y. A. Hong and H. Kang, "Electron tunneling across an interfacial water layer inside an STM junction: tunneling distance, barrier height and water polarization effect," Applied Physics A Materials Science \& Processing, 1998.

[42] M. A. Kozell, "Investigation of the Acoustic Response of a Confined Mesoscopic Water Film Utilizing a Combined Atomic Force Microscope and Shear Force Microscope Technique," 2018. 
[43] G. Heinzel, A. Ru "diger and R. Schilling, "Spectrum and spectral density estimation by the Discrete Fourier transform (DFT), including a comprehensive list of window functions and some new flat-top windows.," Max-Planck-Institut fu $\ddot{r}$ Gravitationsphysik (Albert-Einstein-Institut) Teilinstitut Hannover, 15 Febuary 2002.

[44] L. Sirghi, R. Szoszkiewicz and E. Riedo, "Volume of a Nanoscale Water Bridge," Langmuir, vol. 22, no. 3, pp. 1093-1098, 2006.

[45] R. P. Feynman, R. B. Leighton and M. Sands, The Feynman Lectures on Physics, vol. 2, Reading, Massachusetts: Addison-Wesley, 1965. 


\section{$\underline{\text { Appendix A }}$}

\section{Derivation of the effective spring constant of a cantilever}

The cantilever section in the figure below comprises one half of the tuning fork oscillator. We consider the case where one (or both) of these beams is slightly displaced from equilibrium as shown in (figure left). Assuming uniform bending, we can analyze a small section of beam to determine the internal forces required to exhibit such deformation (figure right). Using curvilinear coordinates, we can define a radial unit vector $\hat{r}$ acting from the center of curvature, and tangential unit vector $\hat{s}$ defined to be positive clockwise. Considering this beam section has unit length $l=R \theta$, tension forces act tangentially along the bottom half of the beam while compression forces act along the top half (figure right). Mid way between the top and bottom of the beam lies a neutral surface. The applied forces deform the beam such that a thin slice of beam located a distance $r$ above the neutral surface will have a length $l-\Delta l=(R-r) \theta$, while a slice located a distance $-r$ below the neutral surface will have corresponding length $l+\Delta l=(R+r) \theta$.
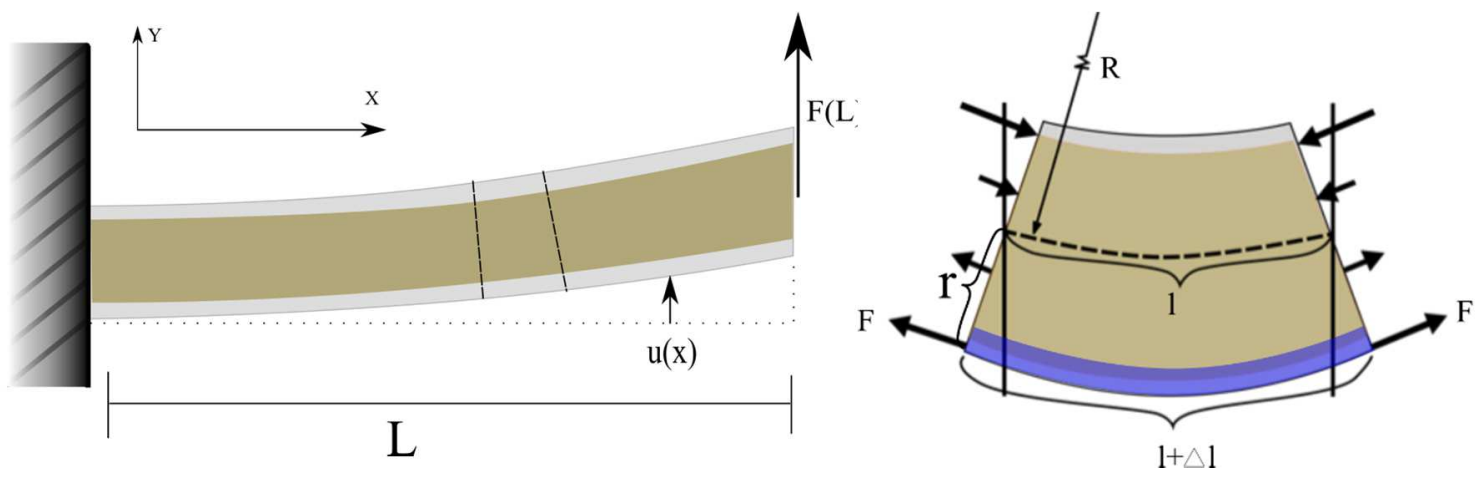

Left) Beam. Right) beam section 
A materials Young's Modulus $E$ is defined in terms of strain in response to an applied stress as

$$
\frac{F}{A}=E \frac{\Delta L}{L}
$$

Given the expressions for $\mathrm{L}$ and $\Delta L$ above, we can rewrite this as $\frac{F}{A}=E \frac{r}{R}$. Taking the thin beam slice to be a differential element we have

$$
d F=\frac{E r}{R} d A
$$

We can now define the bending moment as the torque acting about the neutral surface as

$$
m=\int_{\text {section }}^{\operatorname{Cross}} r d F
$$

from A1.1.2 the bending moment becomes

$$
m=\int_{\text {section }}^{\operatorname{Cross}} \frac{E r^{2}}{R} d A
$$

recognizing $\int r^{2} d A$ as the moment of inertia 'I' we have

$$
m=\frac{I E}{R}
$$

utilizing the definition of radius of curvature in standard Cartesian coordinates

$$
\frac{1}{R}=\frac{\frac{d^{2} u}{d x^{2}}}{\left(1+\frac{d u^{2}}{d x}\right)^{\frac{3}{2}}}
$$

and applying the approximation the deflecting is small meaning $\frac{d u^{2}}{d x} \ll 1$, yields $\frac{1}{R}=\frac{d^{2} u}{d x^{2}}$. Equating 5 now becomes

$$
m=I E \frac{d^{2} u}{d x^{2}}
$$


We can relate this expression to the bending moment about the neutral axis of the total beam.

$$
m=F(L-x)
$$

relating these two expressions and integrating twice

$$
\begin{aligned}
& \frac{d^{2} u}{d x^{2}}=\frac{F}{I E}(L-x) \\
& u(x)=\frac{F}{I E}\left(\frac{L x^{2}}{2}-\frac{x^{3}}{6}\right)
\end{aligned}
$$

Subject to the boundary conditions $u=\frac{d^{2} u}{d x^{2}}=0, @ x=0$. Finally, we return to our definition for moment of inertia $I=\int r^{2} d A$ and calculate explicitly the integral over the thickness $\mathrm{T}$ of the beam section. In the case of the rectangular tuning fork arms, the width is constant

$$
I=\int_{-\frac{T}{2}}^{\frac{T}{2}} r^{2} W d r=\frac{W T^{3}}{12}
$$

Plugging 11 in for 10 , and evaluating for $\mathrm{x}=\mathrm{L}$ returns the cantilever amplitude at the probe location which we are interested in. The result is

$$
u(L)=\frac{4 F}{E W}\left(\frac{L}{T}\right)^{3}
$$

or rearranging in the form of hooks law $F=\frac{E W}{4}\left(\frac{T}{L}\right)^{3} u(L)$, clearly

$$
\mathrm{K}_{\mathrm{eff}}=\frac{E W}{4}\left(\frac{T}{L}\right)^{3} \text {. }
$$




\section{$\underline{\text { Appendix B }}$}

\section{Derivation of the effective mass of a cantilever}

In this section, the approach taken is to look for normal modes of vibration, where the beam (tuning fork tine) undergoes time and space dependent lateral displacement of the form

$$
u(x, t)=u_{0} e^{i \omega t}
$$

We can consider an infinitesimal beam slice where the net force $\mathrm{F}$ is applied between $x$ and $x+d x$. The sum of the forces in the y direction

$$
\sum F_{y}=F(x+d x)-F(x) \cong d x\left(\frac{\partial F(x, t}{\partial x}\right)
$$

If the beam segment is instantaneously moving in the y direction, its y component of velocity and acceleration are:

$$
\begin{aligned}
\dot{u}(x) & =\frac{\partial u}{\partial t}=i \omega u_{0}(x) e^{i \omega t} \\
\text { and } \quad \ddot{u}(x) & =\frac{\partial \dot{u}}{\partial t}=-\omega^{2} u_{0}(x) e^{i \omega t}
\end{aligned}
$$

respectively. The elemental mass of the slice is $d m=d x(\rho A)$, therefore applying Newton's second law we have

$$
d x\left(\frac{\partial F(x, t}{\partial x}\right)+d x(\rho A) \omega^{2} u_{0}(x) e^{i \omega t}=0
$$

Also, from the $\mathrm{z}$ component of the Moment balance equation

$$
\frac{\partial m}{\partial x}+F(x, t)=0
$$

Then, taking the partial derivative of each side with respect to $\mathrm{x}$

$$
\frac{\partial^{2} m}{\partial x^{2}}+\frac{\partial F(x, t)}{\partial x}=0
$$

From equation 5, we can divide through by $d x$, and plug the result into equation 7 . 


$$
\frac{\partial^{2} m}{\partial x^{2}}-(\rho A) \omega^{2} u_{0}(x) e^{i \omega t}=0
$$

In the previous section, we derived $m=I E \frac{d^{2} u}{d x^{2}}$, substituting this expression for the bending moment into equation 8 ,

$$
\frac{\partial^{2}}{\partial x^{2}}\left(E I \frac{\partial^{2} u}{\partial x^{2}}\right)-(\rho A) \omega^{2} u_{0}(x) e^{i \omega t}=0
$$

with the explicit form of $u(x, t)$ from equation 1 this becomes,

$$
u^{\prime \prime \prime \prime}(x)-\frac{\rho A \omega^{2}}{E I} u(x)=0 .
$$

We can introduce a parameter $\beta^{4}=\frac{\rho A \omega^{2}}{E I}$, and express the final form where the primes are recognized to be partial derivatives with respect to $\mathrm{x}$

$$
u^{\prime \prime \prime \prime}(x)-\beta^{4} u(x)=0
$$

Homogeneous solutions to 10 are of the form

$$
u(x)=C_{1} \cosh (\beta x)+C_{2} \sinh (\beta x)+C_{3} \cos (\beta x)+C_{4} \sin (\beta x),
$$

subject to the boundary conditions

$$
\begin{gathered}
u(0)=0 \& u^{\prime}(0)=0 \\
C_{1}+C_{3}=0 \text { or } C_{3}=-C_{1} \\
C_{2}+C_{4}=0 \text { or } C_{4}=-C_{2} .
\end{gathered}
$$

Therefore, the general solution can be expressed as

$$
u(x)=C_{1}(\cosh (\beta x)-\cos (\beta x))+C_{2}(\sinh (\beta x)+\sin (\beta x)) .
$$

Applying the second boundary condition

$$
u^{\prime \prime}(L)=0 \& u^{\prime \prime \prime}(L)=0
$$

This yields the matrix solutions 


$$
\left[\begin{array}{cc}
(\cosh (\beta L)+\cos (\beta L)) & (\sinh (\beta L)+\sin (\beta L)) \\
(\sinh (\beta L)-\sin (\beta L)) & (\cosh (\beta L)+\cos (\beta L))
\end{array}\right]\left\{\begin{array}{l}
C_{1} \\
C_{2}
\end{array}\right\}=\left\{\begin{array}{l}
0 \\
0
\end{array}\right\}
$$

For non-trivial solutions, we require that the determinant is equal to zero

$$
2(1+\cos (\beta L) \cosh (\beta L))=0 .
$$

There are an infinite number of solutions, but the first root corresponding to the fundamental mode occurs where $\alpha=\beta L=1.875104$. From our definition of $\beta$, we can say $(\beta L)^{4}=\frac{\rho A \omega^{2} L^{4}}{E I}$, where $I=\frac{W T^{3}}{12}$, and $\mathrm{K}_{\mathrm{eff}}=\frac{3 E I}{L^{3}}$. Finally, we can express

$$
\omega=\sqrt{\frac{\alpha^{4} \mathrm{~K}_{\mathrm{eff}}}{3 \rho L A}}
$$

where clearly, $\rho L A$ is equal to mass $\mathrm{M}$.

$$
\omega=\sqrt{\frac{K_{\text {eff }}}{0.2427 M}}
$$

Evidently we have found the effective mass $M_{\text {eff }}$ that relates the mass of a cantilever to an equivalent SHO system.

$$
M_{\text {eff }}=0.2427 M
$$




\section{$\underline{\text { Appendix C }}$}

\section{Deriving the Mechanical Amplitude and Phase lag of a Quartz Tuning Fork}

\section{from its Electrical Response.}

The Tuning Forks mechanical displacement can be derived from the electrical response, starting from the BDV model. This ideal circuit is comprised of an RLC circuit modeling the physical oscillator in parallel with a capacitor modeling the internal capacitance. Of course, the current in the leg containing parallel capacitor $I_{c}=A_{c} \sin (\omega t+$ $\pi / 2$ ) will always lead the driving potential with its impedance along the positive imaginary axis. The current $I_{m}=A_{m} \sin \left(\omega t+\theta_{m}\right)$ through the leg containing the RLC circuit (labeled $I_{m}$ because it is the current through the portion of the circuit modeling the mechanical resonator) will lie in the complex plane with phase angle $\theta_{m}$ dependent on frequency. The total current through both legs $I_{e}=\sin \left(\omega t+\theta_{e}\right)$ comprises the real measurable current of the device. The desired $I_{m}$ can be easily calculated from the measured $I_{e}$ by utilizing the law of cosines. 

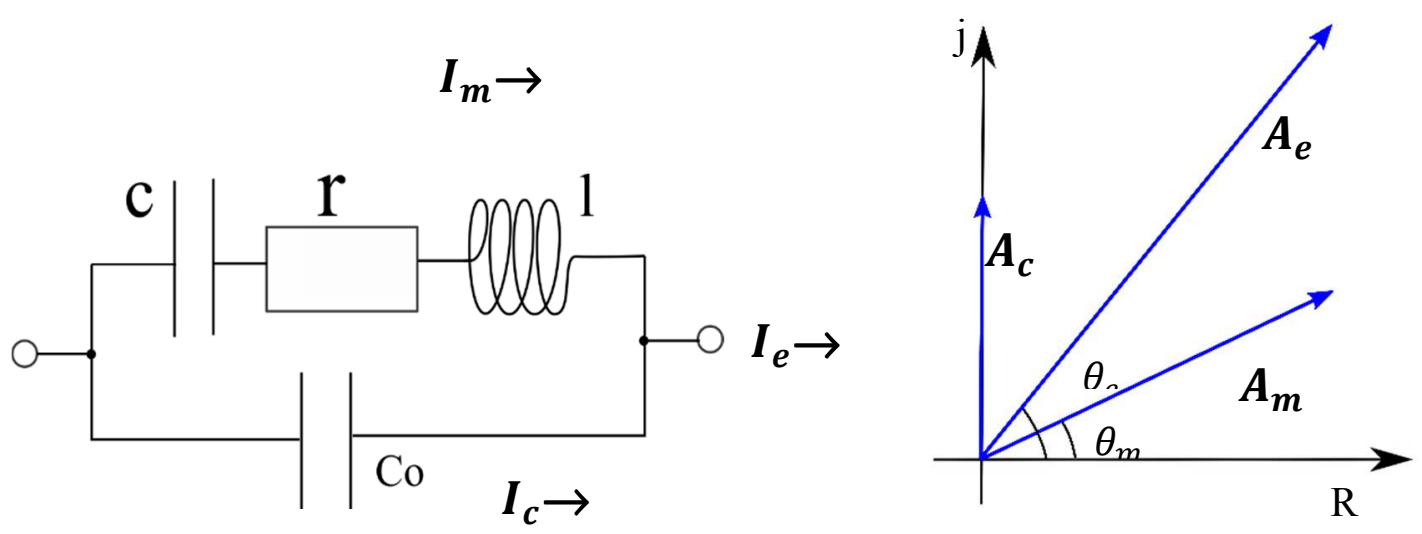

Left) schematic diagram of Butterworth Van Dyke circuit, displaying the current in each leg as well as the total current through the device Right) (above) exaggerated phasor diagram displaying the relative positions of each current contribution. $\theta_{e}$ and $\theta_{m}$ are electrical phase measurements (with respect to driving potential) of the total current through circuit $\boldsymbol{I}_{\boldsymbol{e}}$, and current through the RLC leg $\boldsymbol{I}_{\boldsymbol{m}}$ respectively.

$$
A_{m}^{2}=A_{e}^{2}+A_{c}^{2}-2 A_{e} A_{c} \cos \left(\frac{\pi}{2}-\theta_{e}\right)
$$

or,

$$
A_{m}=\sqrt{A_{e}^{2}+A_{c}^{2}-2 A_{e} A_{c} \sin \theta_{e}}
$$

But the amplitude of the current $A_{c}$ flowing through the capacitor can be calculated as $V_{0} \omega C_{0}$,

$$
A_{m}=\sqrt{A_{e}{ }^{2}+V_{0} \omega C_{0}{ }^{2}-2 A_{e} V_{0} \omega C_{0} \sin \theta_{e}}
$$

Clearly, the phase is then described as,

$$
\tan ^{-1} \theta_{m}=\left(\frac{A_{e} \sin \theta_{e}-V_{0} \omega C_{0}}{A_{e} \cos \theta_{e}}\right) .
$$




\section{$\underline{\text { Appendix D }}$}

\section{Derivation of Tuning Fork -Sample Interaction Forces}

\section{(Case: Frequency Modulation)}

To effectively measure viscoelastic interaction forces using the tuning fork sheer force sensor, a theoretical framework must first be introduced. The laterally oscillating tuning fork can be analyzed in terms of a harmonic oscillator with effective mass $\mathrm{m}_{\text {eff }}$, spring constant $\mathrm{K}_{\mathrm{eff}}$, and internal dissipation $\gamma$, each to be determined later. We begin by describing the probes equation of motion devoid of any interaction forces.

$$
\mathrm{m}_{\mathrm{eff}} \frac{d^{2} u(t)}{d t^{2}}+\mathrm{m}_{\mathrm{eff}} \gamma \frac{d u}{d t}+\mathrm{K}_{\mathrm{eff}} u(t)=F_{\text {ext }}
$$

The tapered probe is driven electromechanically with harmonic motion meaning the driving term can be written more explicitly as $F_{\text {ext }}=F_{0} e^{i \omega t}$. In response, the tapered probes displacement becomes $u(t)=u_{0} e^{i(\omega t+\varphi)}$.

$$
u_{0} e^{i(\omega t+\varphi)}\left[-\omega^{2} \mathrm{~m}_{\mathrm{eff}}+\mathrm{i} \omega \mathrm{m}_{\mathrm{eff}} \gamma+\mathrm{K}_{\mathrm{eff}}\right]=F_{0} e^{i \omega t}
$$

or more compactly,

$$
u_{0} e^{i \varphi}\left[-\omega^{2}+i \omega \gamma+\frac{\mathrm{K}_{\mathrm{eff}}}{\mathrm{m}_{\mathrm{eff}}}\right]=\frac{F_{0}}{\mathrm{~m}_{\mathrm{eff}}}
$$

we rewrite $\frac{\mathrm{K}_{\mathrm{eff}}}{\mathrm{m}_{\mathrm{eff}}}$, as $\omega_{0}^{2}$ the probes resonant frequency (squared). This microscope implements frequency modulation, meaning a phase lock loop detects the probes phase with respect to the driving force, and adjusts the driving frequency attempting always to maintain the probe oscillating at response. Keeping this frequency modulation in mind, equation 3 can be re-expressed as, 


$$
u_{0} e^{i \varphi_{i}} \omega_{0} \gamma=\frac{F_{0}}{\mathrm{~m}_{\mathrm{eff}}}
$$

While the probe is brought into contact with a sample, additional interaction forces may participate, including an additional damping force $\gamma_{i n t} \frac{d u}{d t}$, and elastic force $\mathrm{K}_{\mathrm{int}} u(t)$. Equation (1) becomes,

$$
u_{0}^{\prime} e^{i\left(\omega t+\varphi^{\prime}\right)}\left[-\omega^{\prime 2} \mathrm{~m}_{\mathrm{eff}}+i \omega^{\prime} m_{e q}\left(\gamma+\gamma_{i n t}\right)+\left(\mathrm{K}_{\mathrm{eff}}+\mathrm{K}_{\mathrm{int}}\right)\right]=F_{0} e^{i \omega^{\prime} t}
$$

or,

$$
u_{0}^{\prime} e^{i \varphi^{\prime}}\left[-\omega^{\prime 2}+i \omega^{\prime}\left(\gamma+\gamma_{i n t}\right)+\frac{\left(\mathrm{K}_{\mathrm{eff}}+\mathrm{K}_{\mathrm{int}}\right)}{\mathrm{m}_{\mathrm{eff}}}\right]=\frac{F_{0}}{\mathrm{~m}_{\mathrm{eff}}}
$$

Similarly, $\frac{\left(\mathrm{K}_{\mathrm{eff}}+\mathrm{K}_{\mathrm{int}}\right)}{\mathrm{m}_{\mathrm{eff}}}$ can be re-expressed as ${\omega_{0}}^{\prime 2}$ to thus identify the probe's new resonant frequency that results from the additional elastic interaction. Again, applying the frequency modulation condition, (6) becomes

$$
u_{0}^{\prime} e^{i \varphi^{\prime}} i \omega_{0}^{\prime}\left(\gamma+\gamma_{i n t}\right)=\frac{F_{0}}{m_{\text {eff }}}
$$

Dividing equation (7) by (4):

$$
\frac{u_{0} e^{i \varphi^{\prime}} i \omega_{0}^{\prime}}{u_{0} e^{i \varphi} i \omega_{0}}\left(\frac{\gamma+\gamma_{i n t}}{\gamma}\right)=1
$$

or expressing in terms of frequency linear frequency and re-arranging

$$
\gamma_{i n t}=\gamma\left(\frac{f_{0} u_{0}}{f_{0}^{\prime} u^{\prime}}-1\right)
$$

Yielding the damping force due to interaction as

$$
F_{\text {damping }}=i \omega u_{0}{ }^{\prime} m_{e q} \gamma\left(\frac{f_{0} u_{0}}{f^{\prime} u^{\prime}}-1\right)
$$


The elastic coefficients are simply determined by dividing $\frac{\left(\mathrm{K}_{\mathrm{eff}}+\mathrm{K}_{\mathrm{int}}\right)}{\mathrm{m}_{\mathrm{eff}}}=\omega_{0}{ }^{2}$, by $\frac{\mathrm{K}_{\mathrm{eff}}}{\mathrm{m}_{\mathrm{eff}}}=$ $\omega_{0}^{2}$

$$
\mathrm{K}_{\mathrm{int}}=\mathrm{K}_{\mathrm{eff}}\left(\left(\frac{f_{0}{ }^{\prime}}{f_{0}}\right)^{2}-1\right)
$$

or

$$
F_{\text {elastic }}=u_{0}{ }^{\prime} \mathrm{K}_{\mathrm{eff}}\left(\left(\frac{f_{0}{ }^{\prime}}{f_{0}}\right)^{2}-1\right)
$$




\section{$\underline{\text { Appendix E }}$ \\ Derivation of mesoscopic fluid's damping and elastic coefficients}

\section{(Case: constant frequency)}

The equation of motion describing that tuning forks harmonic motion can be described as

$$
M_{e f f} \ddot{U}+M_{e f f}\left(\gamma_{e f f}+\gamma_{i}\right) \dot{U}+\left(K_{e f f}+K_{i}\right) U=F_{\text {ext }}
$$

Here, the driving force is harmonic of the form $F_{e x t}=F_{a} e^{i \omega t}$. Similarly, harmonic solutions are of the form

$$
\begin{aligned}
& U=U_{a} e^{i(\omega t+\varphi)} \\
& \dot{U}=i \omega U_{a} e^{i(\omega t+\varphi)} \\
& \ddot{U}=-\omega^{2} U_{a} e^{i(\omega t+\varphi)}
\end{aligned}
$$

Plugging in these solutions

$$
\begin{gathered}
U_{a} e^{i \varphi}\left[\frac{\left(K_{e f f}+K_{i}\right)}{M_{e f f}}-\omega^{2}+i \omega\left(\gamma_{e f f}+\gamma_{i}\right)\right]=\frac{F_{e x t}}{M_{e f f}} \\
U_{a} e^{i \varphi}=\left(F_{e x t} / M_{e f f}\right) \frac{\left[\frac{\left(K_{e f f}+K_{i}\right)}{M_{e f f}}-\omega^{2}\right]-i\left[\omega\left(\gamma_{e f f}+\gamma_{i}\right)\right]}{\left[\frac{\left.K_{e f f}+K_{i}\right)}{M_{e f f}}-\omega^{2}\right]^{2}+\left[\omega\left(\gamma_{e f f}+\gamma_{i}\right)\right]^{2}}
\end{gathered}
$$

rewriting in polar form yields the well knows solutions,

$$
U_{a} e^{i \varphi}=\frac{\left(F_{e x t} / M_{e f f}\right) e^{i \varphi}}{\sqrt{\left[\frac{\left(K_{e f f}+K_{i}\right)}{M_{e f f}}-\omega^{2}\right]^{2}+\left[\omega\left(\gamma_{e f f}+\gamma_{i}\right)\right]^{2}}}
$$

where

$$
\tan \varphi=\frac{\omega\left(\gamma_{e f f}+\gamma_{i}\right)}{\left(K_{e f f}+K_{i}\right) / M_{e f f}-\omega^{2}}
$$


here we can make a change of variable

$$
b=\omega\left(\gamma_{e f f}+\gamma_{i}\right), \text { and } a=\frac{\left(K_{e f f}+K_{i}\right)}{M_{e f f}}-\omega^{2}
$$

substituting these in

$$
\begin{aligned}
& U=\frac{\left(F_{\text {ext }} / M_{\text {eff }}\right)}{\sqrt{a^{2}+b^{2}}}, \tan \varphi=\left(\frac{b}{a}\right) \\
& U=\frac{\left(F_{\text {ext }} / M_{\text {eff }}\right)}{\sqrt{a^{2}+a^{2} \tan ^{2} \varphi}} \\
& a=\frac{\left(F_{\text {ext }} / M_{\text {eff }}\right)}{U \sec \varphi}
\end{aligned}
$$

therefore

$$
b=\frac{\left(F_{\text {ext }} / M_{\text {eff }}\right)}{U \sec \varphi} \tan \varphi
$$

substituting back the original variables

$$
\begin{aligned}
& \frac{\left(K_{e f f}+K_{i}\right)}{M_{e f f}}-\omega^{2}=\frac{\left(F_{e x t} / M_{e f f}\right)}{U \sec \varphi} \\
& K_{i}=M_{e f f}\left(\frac{F_{e x t} \cos \varphi}{M_{e f f} U}+\omega^{2}\right)-K_{e f f} \\
& K_{i}=\frac{F_{e x t} \cos \varphi}{U}+M_{e f f}\left(\omega^{2}-\omega_{0}^{2}\right) \\
& K_{i}=\frac{F_{e x t} \cos \varphi}{U}-K_{e f f}\left(1-\frac{\omega^{2}}{\omega_{0}^{2}}\right)
\end{aligned}
$$

and

$$
\omega\left(\gamma_{e f f}+\gamma_{i}\right)=\frac{\left(F_{e x t} / M_{e f f}\right)}{U \sec \varphi} \tan \varphi
$$




$$
\gamma_{i}=\left(\frac{F_{e x t} \sin (2 \varphi)}{2 M_{e f f} U \omega}\right)-\gamma_{e f f}
$$




\section{$\underline{\text { Appendix F }}$}

\section{Calculating Power Spectral Density}

The plots of power spectral density were compiled using the methods outlined in the paper by G. Heinzel, A. Ru diger, and R. Schilling, entitled "Spectrum and spectral density estimation by the Discrete Fourier transform (DFT), including a comprehensive list of window functions and some new flat-top windows" [44]. The details of this analysis will be discussed herein. The data for the noise analysis measurements were collected in the time domain using a digital storage Tektronix oscilloscope (TDS 2024b), connected to a windows computer via Tektronix's OpenChoice desktop software (v2.6) In each case 2500 samples were collected with an acquisition rate $f_{a}$ of $10 \mathrm{KHz}$, putting an upper limit of 5 $\mathrm{KHz}$ on spectral measurements considering Nyquist limitations. The data was converted form the time domain, into the frequency domain using Matlab's DFT

$$
Y(k)=\sum_{j=1}^{n} X(j) e^{\left(\frac{-2 \pi i}{n}\right)(j-1)(k-1)}, 0 \leq k \leq n-1
$$

Where $\mathrm{X}(\mathrm{j})$ is, the data collected in the time domain, and $\mathrm{Y}(\mathrm{k})$ is the same data converted into the frequency domain. Because the collected data only has real components while the DFT is a complex function, only half the outputs are unique meaning,

$$
Y(N-k)=Y^{*}(k)
$$

where $Y^{*}(k)$ is the complex conjugate of $Y(N-k)$ [44].The domain of the DFT's output is divided into a finite number of frequency bins, each with resolution $f_{\text {res }}$ calculated by dividing the acquisition rate by total number of samples, or $4 \mathrm{~Hz}$ in this case. 
Because the dataset is a discrete sample of a larger set containing many superimposed periodic functions, the beginning and end points may cause discontinuities adding spurious peaks in the power spectrum. To visualize this, one can imagine taking the Fourier transform of 1.25 period length sinusoid. There would be a central maximum at waves frequency with successively smaller noise peaks representing spectral leakage radiating on each side of the central maximum. To combat this, a windowing function $w(j)$ is multiplied by $X(j)$ to drive the datasets endpoints to zero creating a continuous function. In this case, the hanging windowing function in Matlab was used where

$$
w(j)=0.5\left(1-\cos \left(2 \pi \frac{j}{N}\right)\right), 0 \leq j \leq N .
$$

The windowing doesn't eliminate the spectral leakage problem; however, it greatly diminishes the amplitude of the noisy peaks. Different windowing functions effect the bandwidth and attenuation of the spectral leakage differently. For this reason, it is advantageous to characterize a parameter called the Effective Noise Bandwidth (ENBW), but before this is done, two normalization sums must be defined,

$$
S_{1}=\sum_{j=0}^{n-1} w(j) \text {, and } S_{2}=\sum_{j=0}^{n-1} w(j)^{2}
$$

With these definitions,

$$
E N B W=f_{a} \frac{S_{2}}{S_{1}{ }^{2}}
$$

In the end, the properly scaled power spectrum can be calculated as,

$$
P S_{\text {rms }}\left(k * f_{\text {res }}\right)=\frac{2 *|Y(k)|^{2}}{S_{1}{ }^{2}},
$$

While the power spectrum density (PSD) $\left(v^{2} / \mathrm{Hz}\right)$, is calculated as, 


$$
P S D_{r m s}\left(k * f_{\text {res }}\right)=\frac{P S_{r m s}\left(k * f_{r e s}\right)}{E N B W}=\frac{2 *|Y(k)|^{2}}{f_{a} * S_{2}}
$$

\section{Reference [43]}

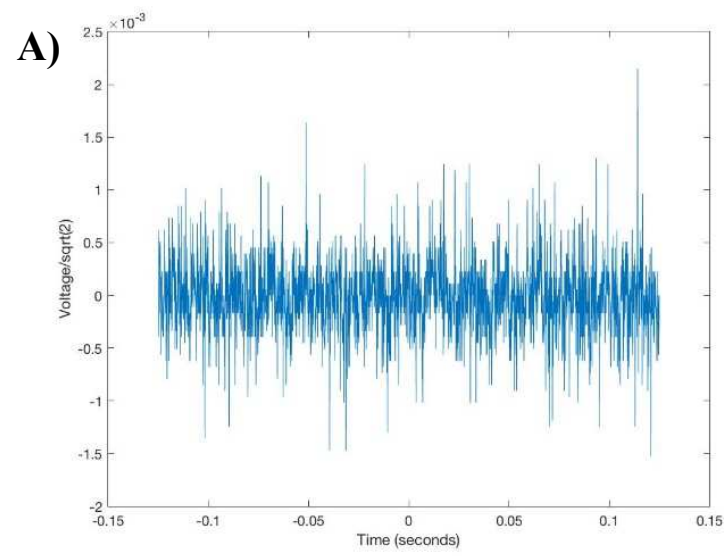

B)
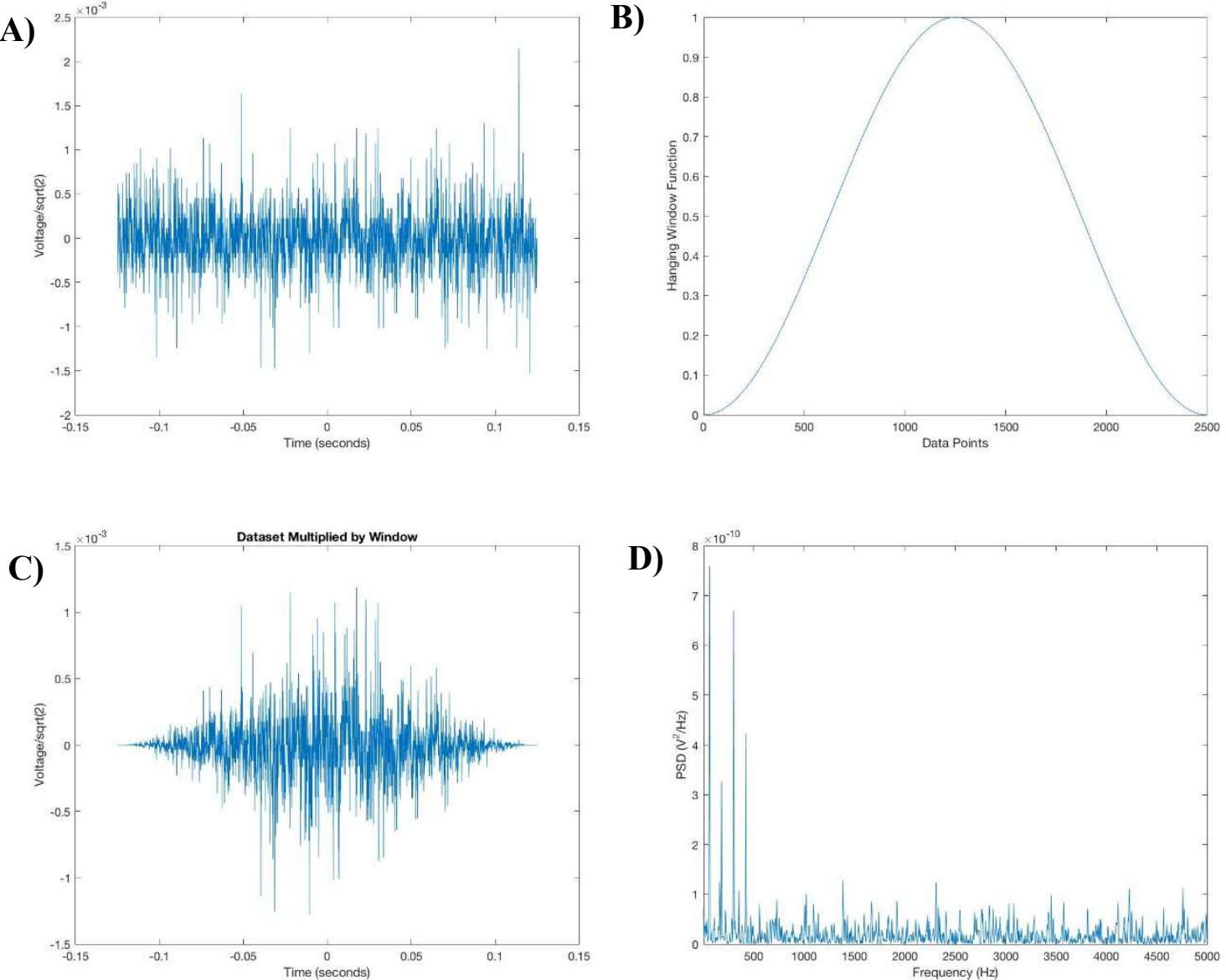

A) The Noise data acquired using the digital storage Tektronix oscilloscope in the time domain, B) Plot of windowing function. C) Plot of windowing function multiplied by dataset. D) Power Spectral Density pot. 


\section{$\underline{\text { Appendix G }}$}

\section{A Note on Synchronous Detection with Lock-In amplifier}

Lock in amplifiers are used to measure extremely small periodic signal, even when distorted by noise that is several orders of magnitude larger. This resolution is achieved by implementing phase sensitive detection. In this method, the lock in utilizes a phase lock loop to create an arbitrary phase internal reference $S i g_{r e f}$ at the same frequency as that used to excite the test signal.

$$
\begin{aligned}
& S i g_{\text {ref }}=A_{\text {ref }} \operatorname{Sin}\left(\omega_{\text {ref }} t+\emptyset_{\text {ref }}\right) \\
& \operatorname{Sig} g_{\text {test }}=A_{\text {test }} \operatorname{Sin}\left(\omega_{\text {test }} t+\emptyset_{\text {test }}\right)
\end{aligned}
$$

The signal under test Sig $_{\text {test }}$ is then amplified to the desired level, digitized, and multiplied by the digitized reference signal, the resulting signal is simply two sinusoids, one at the sum and one at the difference of the two frequencies.

$$
\begin{gathered}
\frac{1}{2} A_{r e f} A_{\text {test }} \operatorname{Sin}\left(\left[\omega_{\text {test }}-\omega_{\text {ref }}\right] t+\emptyset_{\text {test }}-\emptyset_{\text {ref }}\right)- \\
\frac{1}{2} A_{r e f} A_{\text {test }} \operatorname{Sin}\left(\left[\omega_{\text {test }}+\omega_{\text {ref }}\right] t+\emptyset_{\text {test }}+\emptyset_{\text {ref }}\right) .
\end{gathered}
$$

When the reference frequency is equal to that of the test signal $\omega_{\text {test }}=\omega_{\text {ref }}$, the first term becomes constant, and the second term varies at a frequency twice that of the reference. The result is theoretically equal to

$$
\frac{1}{2} A_{\text {ref }} A_{\text {test }} \operatorname{Sin}\left(\emptyset_{\text {test }}-\emptyset_{\text {ref }}\right)
$$

this is highly desirable because it describes both the amplitude and phase of the signal under test this signal is then amplified, and scaled accordingly for a $10 \mathrm{~V}$ system output. 
However, the sinusoidal signal under real test is often obscured with noise that is many orders of magnitude larger. This means that instead of the multiplexed signal being the sum of a DC term with a second oscillating at $2 * \omega_{\text {ref }}$, it will also exhibit oscillatory terms derived by the different noise frequencies. Therefore, great care must be taken during low pass filtering to not only remove the $2 * \omega_{\text {ref }}$ component, but also unwanted noise. The low pass filter bandwidth is defined as,

$$
f_{c}=\frac{1}{2 \pi \tau},
$$

Where $f_{c}$ is the cutoff frequency whereby the signal is attenuated $-3 \mathrm{~dB}$, and $\tau$ is the time constant. While it may initially seem best to minimize the low pass filter bandwidth and eliminate any noise, changes on the filter input take at least 5 time constants to be registered on the output. This delay would restrict the frequency response of the test signal whose amplitude or phase may be varying in time. It can be advantageous then to determine the signals frequency response required by the system and set the low pass filters time constant accordingly.

Another problem that large input noise leads to is high dynamic reserve. Because the test signal is often so small, it needs to be amplified to a value such that the output from the phase sensitive detection (multiplier) can be accurately scaled to the $10 \mathrm{~V}$ output. If, however the noise on top of the test signal is so large that this initial amplification would result in saturation at the Phase sensitive detection; the gain can be reduced on the front end and increased before the output. This large gain to scale the output clearly is unwanted because any offset error or noise will be scaled accordingly. 